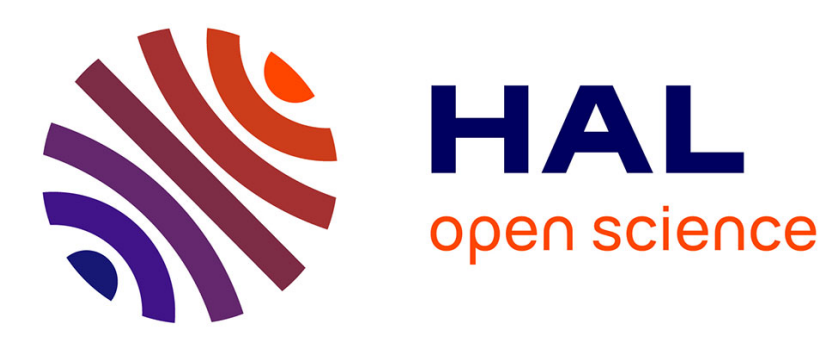

\title{
Uniformisation des variétés de Laumon-Rapoport-Stuhler et conjecture de Drinfeld-Carayol
}

\author{
Thomas Hausberger
}

\section{- To cite this version:}

Thomas Hausberger. Uniformisation des variétés de Laumon-Rapoport-Stuhler et conjecture de Drinfeld-Carayol. Annales de l'Institut Fourier, 2005, 55 (4), pp.1285-1371. 10.5802/aif.2126 . hal00797625

\section{HAL Id: hal-00797625 \\ https://hal.science/hal-00797625}

Submitted on 6 Mar 2013

HAL is a multi-disciplinary open access archive for the deposit and dissemination of scientific research documents, whether they are published or not. The documents may come from teaching and research institutions in France or abroad, or from public or private research centers.
L'archive ouverte pluridisciplinaire HAL, est destinée au dépôt et à la diffusion de documents scientifiques de niveau recherche, publiés ou non, émanant des établissements d'enseignement et de recherche français ou étrangers, des laboratoires publics ou privés. 


\title{
UNIFORMISATION DES VARIÉTÉS DE LAUMON-RAPOPORT-STUHLER ET CONJECTURE DE DRINFELD-CARAYOL
}

\author{
par
}

Thomas Hausberger

\begin{abstract}
Résumé. - Considérons les variétés de « $D$-faisceaux elliptiques » $\mathcal{E} \ell \ell$ introduites par Laumon, Rapoport et Stuhler, définies sur un corps de fonctions $F$ d'une variable sur un corps fini, pour $D$ une algèbre à division de dimension $d^{2}$ sur $F$. Nous montrons que ces variétés admettent, en une place $o$ de $F$ où $D_{o}$ est un corps gauche d'invariant $1 / d$, une uniformisation rigide-analytique par l'espace de Drinfeld $\Omega^{d}$, ou par les revêtements $\Sigma_{n}^{d}$ de $\Omega^{d}$ (selon la structure de niveau). Ce résultat constitue l'analogue du théorème de Čerednik bien connu dans le cas des corps de nombres. Comme application, nous démontrons une conjecture de Carayol : la limite inductive $\Psi_{d}$ suivant $n$ des groupes de cohomologie $\ell$-adique avec support, en degré médian, des revêtements $\Sigma_{n}^{d}-$ sur laquelle agit le produit $\mathrm{GL}_{d}\left(F_{o}\right) \times D_{o}^{*} \times W_{F_{o}}$ - constitue une réalisation géométrique simultanée des correspondances locales de Langlands et de Jacquet-Langlands (du moins pour les cuspidales). Notre preuve est de nature « globale »: via le théorème d'uniformisation, on compare la représentation locale $\Psi_{d}$ à la cohomologie globale de la variété modulaire $\mathcal{E} \ell$.
\end{abstract}

Abstract (Uniformization of Laumon-Rapoport-Stuhler varieties and Drinfeld-Carayol conjecture)

Let us consider Laumon-Rapoport-Stuhler modular varieties $\mathcal{E} \ell \ell$ for " $D$-elliptic sheaves", which are defined over a function field $F$ in one variable over a finite field, for a division algebra $D$ of dimension $d^{2}$ over $F$. We show that these varieties admit, at a place $o$ of $F$ where $D_{o}$ is a skew field of invariant $1 / d$, a rigid-analytic uniformization by Drinfeld's space $\Omega^{d}$, or by the coverings $\Sigma_{n}^{d}$ of $\Omega^{d}$ (depending on the level structure). This result is the analogue of Čerednik's theorem, which is well known in the number field case. As an application, we prove a conjecture of Carayol's : the inductive limit $\Psi_{d}$ over $n$ of the $l$-adic cohomology groups with support, in median degree, of the coverings $\Sigma_{n}^{d}-$ on wich the product $\mathrm{GL}_{d}\left(F_{o}\right) \times D_{o}^{*} \times W_{F_{o}}$ acts - yields a geometrical simultaneous realization of the local Langlands and Jacquet-Langlands correspondences. Our proof is of "global" nature : using the uniformization theorem, we compare the local representation $\Psi_{d}$ to the global cohomology of the moduli variety $\mathcal{E} \ell$.

Table des matières

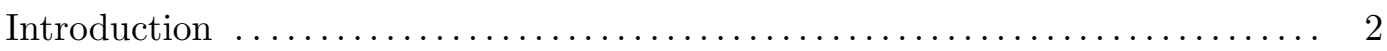

partie I. D-faisceaux elliptiques « spéciaux » $\ldots \ldots \ldots \ldots \ldots \ldots \ldots \ldots$

1. Rappels sur les $\mathcal{D}$-faisceaux elliptiques $\ldots \ldots \ldots \ldots \ldots \ldots \ldots \ldots \ldots \ldots \ldots \ldots$. 6 .

2. Modules divisibles portés par un $\mathcal{D}$-faisceau elliptique $\ldots \ldots \ldots \ldots \ldots \ldots$. 8

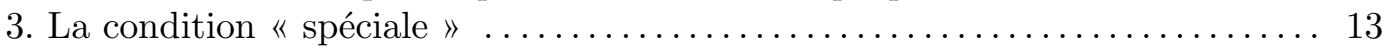

4. Analogue du Théorème de Serre et Tate $\ldots \ldots \ldots \ldots \ldots \ldots \ldots \ldots \ldots \ldots \ldots \ldots . \ldots \ldots$

5. Unicité de la classe d'isogénie ................................. 18

partie II. Uniformisation « à la Čerednik - Drinfeld » ............. 19

6. Le problème de modules des $\mathcal{D}$-faisceaux elliptiques . ................. 19

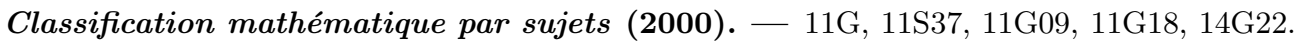




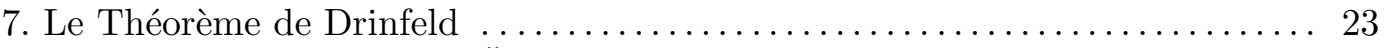

8. Analogue du théorème de Čerednik-Drinfeld ....................... 25

partie III. Conjecture de Drinfeld-Carayol .................... 34

9. Représentations cuspidales dans la cohomologie des revêtements $\Sigma_{n}^{d} \ldots \ldots \ldots 34$

10. Preuve de la conjecture de Drinfeld-Carayol .................... 38

Annexe A. Cohomologie $l$-adique des espaces analytiques, d'après Berkovich .. 51

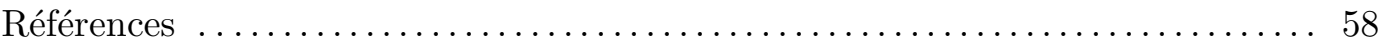

\section{Introduction}

\section{Parties I et II}

Les premiers résultats d'uniformisation $p$-adique remontent à Tate qui introduit dans $[\mathbf{T}]$ la notion d'espace analytique rigide en traitant du cas des courbes elliptiques à réduction multiplicative. Un peu plus tard, Raynaud donne une interprétation de ces espaces rigides-analytiques comme « fibres génériques» de schémas formels (voir [Ra2]). C'est ce point de vue qu'utilise Mumford pour montrer que les courbes de genres quelconques, en certaines places de mauvaise réduction, peuvent être uniformisées par des ouverts convenables du « demi-plan p-adique » $\Omega_{\mathbb{Q}_{p}}^{2}=\mathbb{P}^{1}\left(\widehat{\mathbb{Q}}_{p}\right)-\mathbb{P}^{1}\left(\mathbb{Q}_{p}\right)([\mathbf{M}]$; voir aussi [Ra1 $]$.

Čerednik découvre alors (voir $[\check{\mathbf{C}}]$ ) que tel est le cas de la courbe de Shimura $S$ associée à une algèbre de quaternions $\Delta$ indéfinie de centre $\mathbb{Q}$, en une place $p$ où $\Delta$ est ramifiée : $S \otimes \mathbb{Q}_{p}$ est la réunion de (formes tordues galoisiennes) de quotients à la Mumford de $\Omega_{\mathbb{Q}_{p}}^{2}$ par des sous-groupes de Schottky de $\mathrm{PGL}_{2}\left(\mathbb{Q}_{p}\right)$ (du moins, lorsque l'exposant en $p$ du niveau est maximal). Peu après, Drinfeld en donne une explication naturelle $(\underline{\mathbf{D r} 4})$ : il prouve que $\widehat{\Omega}_{\mathbb{Q}_{p}}^{d} \hat{\otimes} \widehat{\mathbb{Z}}_{p}^{n r}-$ où $\widehat{\Omega}_{\mathbb{Q}_{p}}^{d}$ est le modèle formel de l'analogue $\Omega_{\mathbb{Q}_{p}}^{d}$ en dimension supérieure du demi-plan $p$-adique - est un espace de modules pour des groupes formels, de dimension $d$ et de hauteur $d^{2}$, munis d'une action de l'ordre maximal du corps gauche d'invariant $1 / d$, de centre $\mathbb{Q}_{p}$, et muni d'une « rigidification ». L'uniformisation $p$-adique provient alors de ce qu'en la place $p$ considérée, la courbe de Shimura paramètre des variétés abéliennes toutes isogènes dont le groupe $p$-divisible est de ce type (pour $d=2)$.

La situation qui nous intéresse ici est celle des corps de fonctions rationnelles $F=\mathbb{F}_{q}(X)$ de courbes algébriques $X / \mathbb{F}_{q}$. Désignons par $D$ une algèbre à division de centre $F$ et de rang $d^{2}$, déployée en une place fixée $\infty$ de $X$; on note $\mathcal{R}$ le lieu de ramification de $D$ et on choisit un faisceau d'ordres maximaux $\mathcal{D}$ de $D$. Les variétés qui nous concernent sont les variétés de

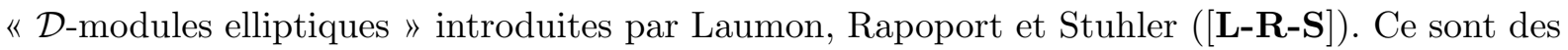
variantes compactes des variétés de modules des faisceaux elliptiques de Drinfeld (voir [Dr1], [Dr2 et Dr3]). Pour tout sous-schéma fini fermé non vide $I$ de $X \backslash\{\infty\}$, les $\mathcal{D}$-faisceaux elliptiques munis d'une $I$-structure de niveau admettent des schémas de modules $\mathcal{E} \ell \ell_{X, \mathcal{D}, I}$ définis sur $X-\{\infty\}-I-\mathcal{R}$, propres et lisses sur $F$ de dimension relative $d-1$.

Considérons une place $o$ de $F$ où $D$ est un corps gauche $D_{o}$ d'invariant $1 / d$. Le théorème que nous démontrons s'énonce comme une uniformisation rigide-analytique :

$$
\left(\mathcal{E} \ell \ell_{X, \mathcal{D}, I}\right)^{a n} \simeq\left[\left(\Omega^{d} \hat{\otimes}_{\mathcal{O}_{o}} \widehat{\mathcal{O}}_{o}^{n r}\right) \times Z_{I}\right] / \mathrm{GL}_{d}\left(F_{o}\right),
$$

où $\mathcal{E} \ell_{X, \mathcal{D}, I}$ désigne le schéma de module obtenu après extension de la définition d'un $\mathcal{D}$-faisceau elliptique (le schéma $\mathcal{E} \ell_{X, \mathcal{D}, I}$ de [L-R-S] n'est pas défini au-dessus de $o$; la bonne définition 
dans notre contexte est celle d'un $\mathcal{D}$-faisceau elliptique « spécial ». La condition « spéciale » est utile afin d'obtenir un schéma raisonnable, c'est-à-dire plat). Précisons bien que le résultat cité est valable uniquement dans le cas où il n'y a pas de structure de niveau en $o$. Dans le cas où l'on met en plus de telles structures, alors on obtient une uniformisation par les revêtements $\Sigma_{n}^{d}$ de $\Omega^{d}$ qu'a construits Drinfeld.

Ce résultat constitue donc l'exact analogue pour les corps de fonctions du théorème de Cerednik-Drinfeld dans le cas des corps de nombres. L'analogie est frappante jusqu'au plan de la preuve elle-même, formellement semblable dans ses grandes lignes à celle que le lecteur peut trouver dans l'article originel de Drinfeld ([Dr4] $)$, ou les dernières pages de son exégèse Bo-Ca par Boutot et Carayol.

Les principaux ingrédients, qui sont réunis dans la partie I, sont les suivants : tout d'abord, il est fondamental de construire le groupe $\pi_{o}$-divisible sous-jacent à un $\mathcal{D}$-faisceau elliptique (défini sur un schéma $S / \mathbb{F}_{q}$ ). Une courbe de Shimura paramétrise une famille de variétés abéliennes dont les complétés formels fournissent ces groupes de manière naturelle. Dans notre contexte, il y a deux constructions du groupe $\pi_{o}$-divisible : la première, directe, utilise le foncteur $G r$ introduit par Drinfeld à propos des variétés de modules des « Stukas»([Dr6]). La seconde, qui passe par l'anti-équivalence de catégorie entre $\mathcal{O}_{o}$-module de Dieudonné et schémas $\pi_{o}$-divisibles en $\mathcal{O}_{o}$-modules, est valable lorsque $S$ est le spectre d'une $\mathcal{O}_{o}$-algèbre locale artinienne. Le groupe est alors construit à partir de la fibre en $o$ du $\mathcal{D}$-faisceau qui possède naturellement la structure d'un $\mathcal{O}_{o}$-module de Dieudonné.

On montre finalement que les deux constructions sont bien équivalentes (pour $S$ convenable). L'utilité principale de cette double construction réside dans la démonstration, plus aisée lorsque l'on manipule des modules de Dieudonné, du théorème de Serre et Tate, ou plutôt de son analogue : déformer un $\mathcal{D}$-faisceau elliptique revient à déformer le $\mathcal{O}_{o}$-module de Dieudonné qu'il porte, donc le module divisible sous-jacent.

En fait, les modules de Dieudonné sont des objets qui interviennent assez naturellement dans la théorie qui nous intéresse. Ils réapparaissent, sous forme de $F_{x}$-modules de Dieudonné, lorsque l'on examine la nature locale de la « fibre générique » d'un $\mathcal{D}$-faisceau elliptique. Leur étude constitue la clef d'un autre ingrédient - capital - de la preuve : les $\mathcal{D}$-faisceaux elliptiques spéciaux sur $\overline{\kappa(o)}$ forment une seule classe d'isogénie.

Nous énonçons puis démontrons l'analogue du théorème de Čerednik-Drinfeld dans la partie II : en gros, on compare les groupes $\pi_{o}$-divisibles obtenus aux groupes formels classifiés par $\widehat{\Omega}^{d} \hat{\otimes}_{\mathcal{O}} \widehat{\mathcal{O}}^{n r}$. Par contre, nous ne dirons rien de la preuve du théorème de Drinfeld, qui en luimême est une pierre maîtresse de l'édifice; nous nous contenterons de substantiels rappels et ferons référence à $[\mathbf{D r 4}], \mathbf{B o}-\mathbf{C a}]$ ou $[\mathbf{G e}$ pour les détails. Signalons la nature particulière de la preuve - valable en égale caractéristique uniquement - que donne Genestier du théorème de Drinfeld : elle fait appel à la théorie du « module de coordonnés » des $\mathcal{O}_{D}$-modules formels, dont nous faisons usage afin de relier modules de Dieudonné et modules divisibles. Peut-être est-il de ce fait possible de « court-circuiter » le théorème de Drinfeld et reconnaître directement dans nos variétés de $\mathcal{D}$-faisceaux elliptiques la trace de l'espace de Drinfeld $\widehat{\Omega}^{d}$, vu à travers l'une appropriée de ses descriptions fonctorielles variées.

\section{Partie III}

Fixons un nombre premier $\ell$, une clôture algébrique $\overline{\mathbb{Q}}_{\ell}$ de $\mathbb{Q}_{\ell}$ (ainsi qu'un isomorphisme $\overline{\mathbb{Q}}_{\ell} \simeq \mathbb{C}$ ), et donnons-nous un corps local non archimédien $K$ (il est donc isomorphe soit à un corps $p$-adique, soit au corps $\mathbb{F}_{q}((t))$ des séries de Laurent formelles en une variable sur un corps fini). Nous notons $\kappa$ le corps résiduel de $K$, de caractéristique $p$ et cardinal $q$. Nous 
désignons par $\mathcal{O}$ l'anneau des entiers de $K$ et par $\varpi$ une uniformisante. Enfin, on s'intéressera aux représentations (définies sur $\overline{\mathbb{Q}}_{\ell}$ ) des trois groupes suivants : $\mathrm{GL}_{d}(K)$, le groupe $D_{d}^{*}$ des éléments inversibles du corps gauche $D_{d}$ sur $K$ d'invariant $1 / d$ (unique à isomorphisme près) et le groupe de Weil $W_{K}$ de $K$ (constitué des éléments de $\operatorname{Gal}(\bar{K} / K)$ qui induisent sur $\kappa$ une puissance entière d'un élément de Frobenius).

La théorie du corps de classes local établit l'existence d'un isomorphisme

$$
C l: W_{K}^{a b} \stackrel{\sim}{\rightarrow} K^{*}=\mathrm{GL}_{1}(K) .
$$

Comme généralisation non-abélienne de ce résultat, Langlands a conjecturé l'existence pour chaque entier $d$ de bijections naturelles $\sigma_{d}$ entre l'ensemble des classes d'équivalence de représentations admissibles irréductibles du groupe $\mathrm{GL}_{d}(K)$ d'une part et l'ensemble des classes d'équivalence de représentations continues semi-simples de dimension $d$ du groupe de Weil $W_{K}$ d'autre part. Cette conjecture a été précisée au fil des ans : les bijections en question doivent être caractérisées par certaines propriétés (voir paragraphe 9.1.2 pour un énoncé précis). L'existence de cette « correspondance de Langlands locale » (pour $\mathrm{GL}_{d}$ ) a été démontrée pour $K=\mathbb{F}_{q}((t))$ par Laumon, Rapoport et Stuhler en 1993 ([L-R-S $]$ ) et, dans le cas des corps $p$-adique, par Harris et Taylor $([\mathbf{H}-\mathbf{T}])$ d'une part, et Henniart ([He2 $]$ ) d'autre part, en 1998 (voir également Ca3 pour une présentation des deux démonstrations).

Langlands a également prédit, avec Jacquet, l'existence d'une seconde famille de bijections, entre l'ensemble des classes d'équivalence de représentations admissibles irréductibles essentiellement de carré intégrable de $\mathrm{GL}_{d}(K)$ d'une part, et l'ensemble des classes d'équivalence de représentations admissibles irréductibles du groupe $D_{d}^{*}$ d'autre part (ils donnent une preuve dans le cas où $d=2$ ). L'existence de cette « correspondance de Jacquet-Langlands locale » (caractérisée également par certaines propriétés : voir paragraphe 9.1.1) a été démontrée en 1984 par Rogawski $([\mathbf{R O}])$, Deligne, Kazhdan et Vignéras $(\mathbf{D}-\mathbf{K}-\mathbf{V}])$ dans le cas des corps $p$-adique (du moins pour les représentations cuspidales) et en 1999 par Badulescu dans le cas d'égale caractéristique $p$ ([Ba1] $)$. En fait, ces deux correspondances ne sont que des cas particuliers parmi les nombreuses fonctorialités prédites par la théorie de Langlands.

Carayol propose dans [Ca2 une réalisation géométrique conjecturale des correspondances locales de Langlands et de Jacquet-Langlands : il définit deux représentations (dites "représentations locales fondamentales ») $\mathcal{U}_{d}^{v}$ et $\mathcal{U}_{d}^{r}$ du groupe produit $\mathrm{GL}_{d} \times D_{d}^{*} \times W_{K}$, provenant respectivement de la cohomologie de variétés de cycles évanescents $\ell$-adiques (associés par la théorie de Berkovich à des schémas formels issus des espaces de déformations des $\mathcal{O}$-modules formels introduits par Drinfeld) et de la cohomologie $\ell$-adique à support compact (définie par Berkovich) de revêtements rigides-analytiques $\Sigma_{n}^{d}$ (définis par Drinfeld dans [Dr1]) du « demiplan de Poincaré généralisé $» \Omega_{K}^{d}$. En fait, la représentation $\mathcal{U}_{d}^{v}$ a été considérée tout d'abord par Deligne (vers 1970) dans le cas $d=2$ et $K=\mathbb{Q}_{p}$, puis étudiée par Carayol, toujours pour $\mathrm{GL}_{2}$, dans le cas des corps $p$-adiques quelconques. De son côté, Drinfeld suggère dans l'introduction de [Dr1] que toute représentation supercuspidale de $\mathrm{GL}_{n}(K)$ est réalisée dans la cohomologie des revêtements galoisiens $\Sigma_{n}^{d}$. Carayol précise ces idées dans son article Ca2] sous la forme d'une conjecture, que nous appellerons « conjecture de Drinfeld-Deligne-Carayol » (plus exactement, conjecture de « Drinfeld-Carayol » dans le cas rigide-analytique, et conjecture de «Deligne-Carayol » dans le cas des cycles évanescents). Cette conjecture affirme que, pour $\pi$ une représentation cuspidale de $\mathrm{GL}_{d}(K)$, les composantes $\pi$-isotypiques des représentations 
locales fondamentales doivent être données par :

$$
\begin{gathered}
\mathcal{U}_{d}^{v}(\pi)=\mathrm{JL}\left(\pi^{\vee}\right) \otimes\left(\sigma_{d}\left(\pi^{\vee}\right) \otimes||^{\frac{1-d}{2}}\right), \\
\mathcal{U}_{d}^{r}(\pi)=\mathrm{JL}(\pi) \otimes\left(\sigma_{d}(\pi) \otimes||^{\frac{1-d}{2}}\right) .
\end{gathered}
$$

Pour résumer les choses : l'une et l'autre des deux représentations locales fondamentales $\mathcal{U}_{d}^{v}$ et $\mathcal{U}_{d}^{r}$ doivent constituer une construction géométrique simultanée des correspondances de Langlands et de Jacquet-Langlands locales pour les cuspidales (noter toutefois la légère différence entre les formules précédentes). Ce qu'il advient des autres représentations (en particulier des séries discrètes non cuspidales) n'est pas encore clair. Toutefois, le lecteur pourra trouver dans [S-S] un calcul complet de la cohomologie de l'espace $\Omega^{d}$. Par ailleurs, la relation entre les représentations $\mathcal{U}_{d}^{v}$ et $\mathcal{U}_{d}^{r}$ vient d'être éclaircie par Faltings (voir [Fa1] et [Fa2]) : parce que les deux prorevêtements deviennent isomorphes après quelques éclatements appropriés, on a bien $\mathcal{U}_{d}^{v}(\pi)=$ $\mathcal{U}_{d}^{r}\left(\pi^{\vee}\right)$ (le « morphisme de périodes » change de sens, d'où le passage à la contragrédiente).

La partie III est consacré à la preuve du quatrième volet (manquant à ce jour) de la conjecture de Drinfeld-Deligne-Carayol : le cas où $K$ est d'égale caractéristique $p$, versant rigide-analytique (c'est-à-dire relatif à $\mathcal{U}_{d}^{r}$ ). Le tableau suivant résume les contributions à la preuve de la conjecture, sous ses quatre aspects :

\begin{tabular}{||l||c|c||}
\hline & versant rigide analytique & versant cycles-évanescents \\
\hline \hline corps $p$-adiques & Harris $([\mathbf{H} \mathbf{1}] ; 1997)$ & $\begin{array}{l}\text { Harris-Taylor } \\
([\mathbf{H}-\mathbf{T}] ; 1998)\end{array}$ \\
& complété par Harris-Taylor $([\underline{\mathbf{H}-\mathbf{T}}])$ & Boyer \\
\hline cas d'égale & Hausberger & $([\mathbf{B o y}] ; 1998)$ \\
caractéristique $p$ & $(2000)$ & \\
\hline
\end{tabular}

Détaillons un peu plus le cas qui nous concerne : notant

$$
\Psi_{d, n}^{i}=\mathrm{H}_{c}^{i}\left(\left(\operatorname{Res}_{\widehat{K}^{n r} / K}^{\prime} \Sigma_{n}^{d}\right)_{\bar{K}}, \overline{\mathbb{Q}}_{\ell}\right)
$$

(où Res $\widehat{K}_{\widehat{K}^{n r} / K}$ désigne la restriction des scalaires « à la Weil », au sens du paragraphe 9.2), la représentation locale fondamentale $\mathcal{U}_{d}^{r}$ est la limite inductive notée désormais

$$
\Psi_{d}^{d-1}=\underset{n}{\lim } \Psi_{d, n}^{d-1}
$$

(cohomologie en degré médian $d-1$ ). On vérifie qu'elle est munie d'une action (admissible(1)) du produit $\mathrm{GL}_{d}(K) \times D_{d}^{*} \times W_{K}$. La preuve de la conjecture de Drinfeld-Deligne-Carayol relative à $\Psi_{d}^{d-1}$ procède par voie globale : on utilise le théorème d'uniformisation, précédemment établi (partie II), des variétés de modules $\mathcal{E} \ell_{X, \mathcal{D}, I}$, et la méthode (inventée par Deligne ([De $]$ ) et développée par différents auteurs (voir [Ca2, [H1], [H-T], Boy])) de comparaison entre la représentation locale $\Psi_{d}$ et la cohomologie globale de la variété modulaire $\mathcal{E} \ell_{X, \mathcal{D}, I}$.

Le plan de cette dernière partie est le suivant : le premier paragraphe est consacré à la présentation de la conjecture de Drinfeld-Carayol, qui est énoncée après quelques rappels sur les correspondances locales de Langlands et de Jacquet-Langlands. Le second paragraphe est de nature globale : il est dédié à la cohomologie $\ell$-adique des variétés $\mathcal{E} \ell_{X, \mathcal{D}, I}$. On y résume les résultats établis par Laumon, Rapoport et Stuhler dans [L-R-S], notamment la description de la cohomologie en rapport avec la correspondance de Langlands locale. La conjecture de Drinfeld-Carayol est démontrée au troisième paragraphe : on construit la suite spectrale de Hochschild-Serre, on montre que sa partie cuspidale est dégénérée, puis on conclut. Enfin, il

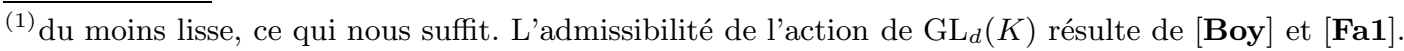


figure en appendice une brève exposition de la théorie cohomologique ( $\ell$-adique) des espaces analytiques de Berkovich. On y démontre notamment un théorème de lissité de l'action sur les groupes de cohomologie à support propre, dû à Berkovich, et établit quelques résultats utilisés lors de la construction de la suite spectrale mentionnée plus haut.

Remerciements : ma reconnaissance envers mes deux directeurs de thèse, Henri Carayol et Thomas Zink, est infinie. Je remercie également Vladimir Berkovich qui m'a transmis, expliqué et autorisé à reproduire ici quelques notes non publiées ; outre sa très belle théorie, ses commentaires ont été inestimables. Enfin, le référee a largement contribué à la concision de ce texte.

\section{Première PARTIE I D-FAISCEAUX ELLIPTIQUES « SPÉCIAUX »}

\section{Rappels sur les $\mathcal{D}$-faisceaux elliptiques}

1.1. Notations. - Dans tout ce qui suit, $X$ désigne une courbe algébrique lisse, projective et géométriquement connexe sur le corps $\mathbb{F}_{q}$ (avec $q$ une puissance $p^{e}$ d'un nombre premier $p$ ). On note $F=\mathbb{F}_{q}(X)$ le corps des fonctions rationnelles sur $X$. Pour tout point fermé $x \in|X|$ (correspondant à une place de $F$ ), on désigne par $\mathcal{O}_{x}$ et $F_{x}$ les complétés respectifs de $\mathcal{O}_{X, x}$ et $F$ en $x$. Le corps résiduel de $\mathcal{O}_{x}$ est noté $\kappa(x)$, de cardinal $q_{x}=q^{\operatorname{deg}(x)}$. La valuation $v_{x}: F_{x} \rightarrow \mathbb{Z}$ est normalisée par la condition $v_{x}\left(\pi_{x}\right)=1$, pour $\pi_{x}$ une uniformisante de $\mathcal{O}_{x}$; la valeur absolue ||$_{x}$ est $q_{x}^{-v_{x}()}$.

On fixe une telle place $\infty$ que l'on supposera, pour simplifier, rationnelle sur le corps des constantes $\mathbb{F}_{q}$ de $X$ (i.e. $\left.\operatorname{deg}(\infty)=1\right)$; on note $A=\Gamma\left(X-\{\infty\}, \mathcal{O}_{X}\right)$. Donnons-nous ensuite une algèbre centrale simple $D$ de dimension $d^{2}$ sur son centre $F$, déployée en $\infty$ (telle que $\left.D \otimes_{F} F_{\infty} \simeq M_{d}\left(F_{\infty}\right)\right)$. On choisit également un faisceau cohérent $\mathcal{D}$ localement libre en $\mathcal{O}_{X^{-}}$ algèbres, de fibre générique $D$, tel qu'en tout point fermé $x$ de $X, \mathcal{D}_{x}=\mathcal{D} \otimes_{\mathcal{O}_{X}} \mathcal{O}_{x}$ soit un ordre maximal de $D_{x}=D \otimes_{F} F_{x}$. On note $\mathcal{R}$ l'ensemble des mauvaises places, où l'algèbre $D_{x}$ n'est pas déployée : pour $x \notin \mathcal{R}$, le couple $\left(D_{x}, \mathcal{D}_{x}\right)$ est donc isomorphe à $\left(M_{d}\left(F_{x}\right), M_{d}\left(\mathcal{O}_{x}\right)\right)$.

Tous les schémas considérés sont des schémas sur $\mathbb{F}_{q}$; on notera $S \times T$ le produit sur $\mathbb{F}_{q}$.

1.2. Définition. - La définition que donnent Laumon, Rapoport et Stuhler d'un $\mathcal{D}$-faisceau elliptique est la suivante :

Définition 1.1. - Soit $S$ un $\mathbb{F}_{q}$-schéma. Un $\mathcal{D}$-faisceau elliptique (de pôle $\infty$ et zéro $z$ ) sur $S$ consiste en la donnée d'un diagramme commutatif :

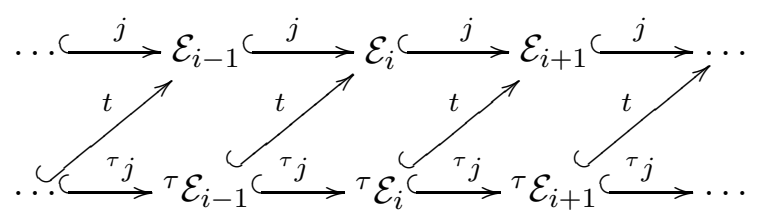

où pour chaque $i \in \mathbb{Z}, \mathcal{E}_{i}$ est un $\mathcal{O}_{X \times S}$-module localement libre de rang $d^{2}$, muni d'une action à droite de $\mathcal{D}$ compatible à l'action de $\mathcal{O}_{X}$, où

$$
{ }^{\tau} \mathcal{E}_{i}=\left(\operatorname{Id}_{X} \times \operatorname{Frob}_{S / \mathbb{F}_{q}}\right)^{*} \mathcal{E}_{i}
$$


(pull-back par le Frobenius de $S$ ), et où les $j$ et $t$ sont des injections $\mathcal{O}_{X \times S^{-}}$-linéaires compatibles à l'action de $\mathcal{D}$. Ces données sont astreintes à satisfaire aux conditions suivantes :

(i) Périodicité : $\mathcal{E}_{i+d}=\mathcal{E}_{i}(\{\infty\} \times S)$, le composé de $d$ morphismes $j$ consécutifs étant l'injection naturelle (induite par $\mathcal{O}_{X} \hookrightarrow \mathcal{O}_{X}(\infty)$ ).

(ii) Pôle : $\mathcal{E}_{i} / j\left(\mathcal{E}_{i-1}\right)$ est isomorphe comme $\mathcal{O}_{X \times S}$-module à l'image directe $\left(\Gamma_{\infty}\right)_{*} \mathcal{A}_{i}$ d'un $\mathcal{O}_{S}$-module $\mathcal{A}_{i}$ localement libre de rang $d$ par la section $\infty$ :

$$
\Gamma_{\infty}: S \rightarrow X \times S, \quad s \mapsto(\infty, s) .
$$

(iii) Zéro : $\mathcal{E}_{i} / t\left({ }^{\tau} \mathcal{E}_{i-1}\right)$ est isomorphe comme $\mathcal{O}_{X \times S}$-module à l'image directe $\left(\Gamma_{z}\right)_{*} \mathcal{B}_{i}$ d'un $\mathcal{O}_{S}$-module $\mathcal{B}_{i}$ localement libre de rang $d$ par une section $\Gamma_{z}: S \rightarrow X \times S$, graphe d'un

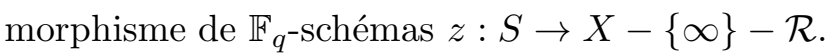

(iv) Normalisation : Pour tout point géométrique $s$ de $S$, la caractéristique d'Euler-Poincaré $\chi\left(\left.\mathcal{E}_{0}\right|_{X \times s}\right)$ vérifie $\chi\left(\left.\mathcal{E}_{0}\right|_{X \times s}\right) \in[0, d[$.

Un isomorphisme entre deux faisceaux elliptiques $\left(\mathcal{E}_{i}, j, t\right)$ et $\left(\mathcal{E}_{i}^{\prime}, j^{\prime}, t^{\prime}\right)$ consiste en un système d'isomorphismes $\psi_{i}: \mathcal{E}_{i} \stackrel{\sim}{\rightarrow} \mathcal{E}_{i}^{\prime}$ de faisceaux en $\mathcal{O}_{X \times S}$-modules, compatible à l'action de $\mathcal{D}$ et tel que, avec les pull-back ${ }^{\tau} \psi_{i}:{ }^{\tau} \mathcal{E}_{i} \stackrel{\sim}{\rightarrow} \tau \mathcal{E}_{i}^{\prime}$, tous les diagrammes commutent.

Remarque. - En fait, dans l'article [L-R-S], la condition de normalisation n'est pas imposée directement : on considère plutôt l'action naturelle de $\mathbb{Z}$, par décalage, sur le champ classifiant les $\mathcal{D}$-faisceaux elliptiques, puis on passe au quotient. Bien sûr, cela revient au même.

1.3. Structures de niveau. - Soit $I \neq \emptyset$ un sous-schéma fermé fini de $X-\{\infty\}$; soit d'autre part $\left(\mathcal{E}_{i}, j, t\right)$ un $\mathcal{D}$-faisceau elliptique sur un schéma $S$, tel que l'image $z(S)$ du morphisme zéro associé soit disjointe de $I$. Dans ces conditions, la restriction $\mathcal{E}_{I}=\left.\mathcal{E}_{i}\right|_{I \times S}$ est indépendante (via $j$ ) de $i$, et $t$ induit un isomorphisme ${ }^{\tau} \mathcal{E}_{I} \simeq \mathcal{E}_{I}$.

On définit alors une structure de niveau $I$ sur $\left(\mathcal{E}_{i}, j, t\right)$ comme la donnée d'un isomorphisme $\mathcal{O}_{I \times S}$-linéaire $\iota: \mathcal{D}_{I} \otimes \mathcal{O}_{S} \simeq \mathcal{E}_{I}$, compatible à l'action à droite de $\mathcal{D}_{I}$ sur les deux membres, et rendant commutatif le diagramme suivant :

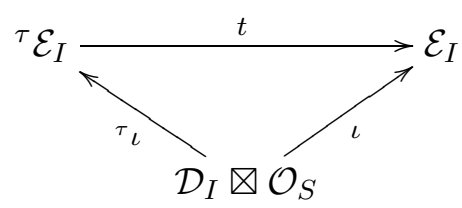

Remarque. - Si $z(S) \cap I \neq \emptyset$, avec cette définition, il n'existe pas de I-structure de niveau car $\left.t\right|_{I \times S}$ n'est plus un isomorphisme. Il est possible cependant d'étendre la définition d'une I-structure de niveau aux niveaux divisant la caractéristique, en disant ce qu'est une "base de Drinfeld" (voir [Dr4]). Lorsque I est disjoint de $\mathcal{R}$, ce travail a été réalisé par Boyer (voir $\mathbf{B o y}$ ). Or cela pose problème aux points où l'algèbre $D$ est ramifiée; c'est pourquoi nous n'aurons pas usage de cette notion dans ce texte.

1.4. Extension de la définition d'un $\mathcal{D}$-faisceau elliptique. - En imposant la condition sur le zéro (le morphisme $z$ se factorise à travers $X-\{\infty\}-I-\mathcal{R}$ ), Laumon, Rapoport et Stuhler évitent les problèmes liés à la mauvaise réduction. Ici au contraire, notre but est d'étudier la réduction en une place $o$ appartenant au lieu de ramification $\mathcal{R}$ de l'algèbre $D$, obtenant ainsi une situation analogue à la mauvaise réduction dans le cas des corps de nombres. Précisément, on suppose que $D_{o}$ est un corps gauche d'invariant $1 / d$ sur $F_{o}$. Il s'agit donc d'étendre la définition d'un $\mathcal{D}$-faisceau elliptique qui est donnée dans [L-R-S]. Une première approche, la plus naïve, consiste à modifier la condition (iii) comme suit : 
(iii') Zéro : $\mathcal{E}_{i} / t\left({ }^{\tau} \mathcal{E}_{i-1}\right)$ est isomorphe comme $\mathcal{O}_{X \times S}$-module à l'image directe $\left(\Gamma_{z}\right)_{*} \mathcal{B}_{i}$ d'un $\mathcal{O}_{S}$-module $\mathcal{B}_{i}$ localement libre de rang $d$ par une section $\Gamma_{z}: S \rightarrow X \times S$, graphe d'un

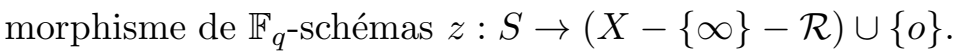

Toutefois, cela n'est guère satisfaisant : afin d'obtenir un schéma de modules raisonnable (en l'occurrence plat), il s'avère nécessaire d'ajouter une condition supplémentaire en la place $o$, dite « condition spéciale », qui sera énoncée ultérieurement. Il s'agit tout d'abord d'expliquer la construction du groupe $\pi_{o}$-divisible $G r_{o}(\mathcal{E})$ que l'on associe à un $\mathcal{D}$-faisceau elliptique $\left(\mathcal{E}_{i}, j, t\right)$ et qui intervient dans l'énoncé de la condition «spéciale ».

\section{Modules divisibles portés par un $\mathcal{D}$-faisceau elliptique}

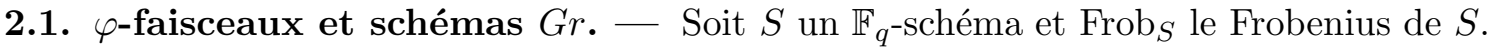

Définition 2.1 (cf. [Dr6] §2). - Un $\varphi$-faisceau sur $S$ est un faisceau $\mathcal{F}$ en $\mathcal{O}_{S}$-module, localement libre de rang fini, et muni d'une application $\mathcal{O}_{S}$-linéaire $\varphi:$ Frob $_{S}^{*} \mathcal{F} \rightarrow \mathcal{F}$.

Soit $\mathbb{V}(\mathcal{F})$ le fibré vectoriel (géométrique) associé au faisceau dual $\mathcal{F}^{*}=\mathcal{H} m_{\mathcal{O}_{S}}\left(\mathcal{F}, \mathcal{O}_{S}\right)$, c'est-à-dire :

$$
\mathbb{V}(\mathcal{F})=\operatorname{Spec}\left(\operatorname{Sym}_{\mathcal{O}_{S}}(\mathcal{F})\right)
$$

D'une part, le morphisme $\varphi$ induit une application $\mathcal{O}_{S}$-linéaire $\mathbb{V}(\mathcal{F}) \rightarrow \operatorname{Frob}_{S}^{*} \mathbb{V}(\mathcal{F})$, que nous noterons $\mathbb{V}(\varphi)$; d'autre part, on a le Frobenius $\operatorname{Frob}_{\mathbb{V}(\mathcal{F})}: \mathbb{V}(\mathcal{F}) \rightarrow$ Frob $_{S}^{*} \mathbb{V}(\mathcal{F})$ qui est $q$-linéaire. Suivant Drinfeld (voir [Dr6]), on définit alors :

Définition 2.2. - Le $S$-schéma $G r(\mathcal{F})$ est le fibré en $\mathbb{F}_{q}$-vectoriels

$$
\operatorname{Gr}(\mathcal{F})=\operatorname{Ker}\left(\mathbb{V}(\varphi)-\operatorname{Frob}_{\mathbb{V}(\mathcal{F})} .\right.
$$

Concrètement, pour tout $S$-schéma $T \stackrel{\alpha}{\rightarrow} S$, on a :

$$
\operatorname{Gr}(\mathcal{F})(T)=\left\{h \in \operatorname{Hom}_{\mathcal{O}_{S}}\left(\mathcal{F}, \alpha_{*} \mathcal{O}_{T}\right), \quad h(\varphi(1 \otimes x))=h(x)^{q} \quad \forall x \in \mathcal{F}\right\} .
$$

Le foncteur $G r$ est un foncteur contravariant de la catégorie des $\varphi$-faisceaux sur $S$ dans la catégorie des schémas en groupes sur $S$ munis d'une action de $\mathbb{F}_{q}$. Donnons quelques propriétés de ce foncteur :

Proposition 2.3 (cf. Dr6, proposition 2.1). - 1) Pour tout $\varphi$-faisceau $\mathcal{F}$ sur $S$, le schéma $\operatorname{Gr}(\mathcal{F})$ est fini et localement libre; si $\mathcal{F}$ est de rang $n$, alors $\operatorname{Gr}(\mathcal{F})$ est d'ordre égal à $q^{n}$.

2) Le faisceau $\operatorname{Lie}^{*} \operatorname{Gr}(\mathcal{F})$ (i.e. l'image inverse du faisceau des différentielles $\Omega_{G r(\mathcal{F}) / S}^{1}$ relativement à la section nulle $O_{S}: S \rightarrow G r(\mathcal{F})$ ) est canoniquement isomorphe au conoyau du morphisme $\varphi:$ Frob $_{S}^{*} \mathcal{F} \rightarrow \mathcal{F}$.

3) Le schéma $\operatorname{Gr}(\mathcal{F})$ est étale au-dessus de $S$ si et seulement si $\varphi$ est un isomorphisme.

4) Le foncteur $G r$ est exact.

5) Le foncteur $\mathrm{Gr}$ est pleinement fidèle.

2.2. Application aux $\mathcal{D}$-faisceaux elliptiques. - La construction qui suit fournit, pour un $\mathcal{D}$-faisceau elliptique, l'analogue du groupe $p$-divisible $A_{p} \infty$ d'une variété abélienne $A$ ( $o$ est l'analogue de $p)$.

Soit $\left(\mathcal{E}_{i}, j, t\right)$ un $\mathcal{D}$-faisceau elliptique défini sur un $\mathbb{F}_{q}$-schéma $S$ et de zéro $z$. On fixe une place $o \neq \infty$ de $X$; le corps résiduel $\kappa(o)$ est de cardinal $q_{o}=q^{\operatorname{deg}(o)}$. Comme $o$ est distinct du pôle, le $\mathcal{O}_{o} \otimes \mathcal{O}_{S}$-module $\mathcal{E}_{i} \otimes \mathcal{O}_{o}$ localement libre de rang $d^{2}$ est, via le morphisme $j$, indépendant de l'indice $i$. On le note $\mathcal{E}_{o}$ et on définit également $\mathcal{E}_{o, n}=\mathcal{E}_{o} \otimes_{\mathcal{O}_{o}} \mathcal{O}_{o} / \mathfrak{m}_{o}^{n}$ pour tout entier $n$. L'image 
directe de $\mathcal{E}_{o, n}$ par la projection $\operatorname{Spec}\left(\mathcal{O}_{o} / \mathfrak{m}_{o}^{n}\right) \times S \rightarrow S$, munie de l'application $\mathcal{O}_{S^{-}}$-linéaire induite par $t_{o}$, est alors un $\varphi$-faisceau sur $S$ que l'on notera encore $\left(\mathcal{E}_{o, n}, t_{o}\right)$.

Définition 2.4. - Soit $\operatorname{Gr}\left(\mathcal{E}_{o, n}\right)$ le $S$-schéma associé au $\varphi$-faisceau $\left(\mathcal{E}_{o, n}, t_{o}\right)$ par le foncteur $\operatorname{Gr}$ du paragraphe précédent. Le groupe $\pi_{o}$-divisible associé au $\mathcal{D}$-faisceau elliptique $\left(\mathcal{E}_{i}, j, t\right)$ est alors :

$$
G r_{o}(\mathcal{E})=\underset{n}{\lim } \operatorname{Gr}\left(\mathcal{E}_{o, n}\right) .
$$

Les propriétés suivantes de l'ind-schéma $G r_{o}(\mathcal{E})$ résultent directement de la proposition 2.3 :

Proposition 2.5. - Pour tout entier $n \geq 1$, le $S$-schéma $\operatorname{Gr}\left(\mathcal{E}_{o, n}\right)$ est un schéma fini en $\mathcal{O}_{o}$-modules qui vérifie :

(i) il existe un entier $N \geq 0$ tel que, en tant que $S$-schéma en $\kappa(o)$-espaces vectoriels $(\kappa(o) \subset$ $\left.\mathcal{O}_{o}\right)$, le schéma $\operatorname{Gr}\left(\mathcal{E}_{o, n}\right)$ peut, localement pour la topologie étale sur $S$, s'injecter dans $\mathbb{G}_{a}^{N}$,

(ii) comme $S$-schéma en $\kappa(o)$-espaces vectoriels, la dimension de $\operatorname{Gr}\left(\mathcal{E}_{o, n}\right)$ est $n d^{2}$ (le schéma en groupes $\operatorname{Gr}\left(\mathcal{E}_{o, n}\right)$ est d'ordre $\left.q_{o}^{n d^{2}}\right)$,

(iii) la suite de $S$-schémas en $\mathcal{O}_{o}$-modules

$$
0 \longrightarrow G r\left(\mathcal{E}_{o, n}\right) \stackrel{i_{n}}{\longrightarrow} G r\left(\mathcal{E}_{o, n+1}\right) \stackrel{\pi_{o}^{n}}{\longrightarrow} \operatorname{Gr}\left(\mathcal{E}_{o, n+1}\right)
$$

est exacte.

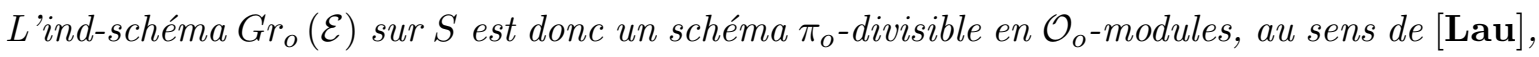
définition 2.4.8.

Notons que $G r_{o}(\mathcal{E})$ est également muni d'une action de $\mathcal{D}_{o}$ qui prolonge celle de $\mathcal{O}_{o}$, puisque le morphisme $\varphi=t_{o}$ est compatible à l'action de $\mathcal{D}_{o} \operatorname{sur} \mathcal{E}_{o}$.

La proposition suivante précise la nature du groupe $\pi_{o}$-divisible $G r_{o}(\mathcal{E})$ en toute place qui ne provient pas de la caractéristique $o$ :

Proposition 2.6. - $G r_{o}(\mathcal{E})$ est ind-étale au-dessus de $S \backslash z^{-1}(\{o\})$.

En effet, le morphisme $t_{o}:{ }^{\tau} \mathcal{E}_{o} \otimes \kappa(s) \rightarrow \mathcal{E}_{o} \otimes \kappa(s)$ est un isomorphisme dès que $z(s) \neq o$; c'est alors un corollaire immédiat de la proposition 2.33 ).

Au contraire, on verra plus loin que $G r_{o}(\mathcal{E})$ est infinitésimal en $o$ (du moins lorsque $\left(\mathcal{E}_{i}, j, t\right)$ vérifie la condition « spéciale »).

Expliquons pour finir comment la notion de structure de niveau peut se traduire à l'aide du $G r_{o}$. Dans la situation qui nous intéresse, $D_{o}$ est un corps gauche d'invariant $1 / d$ sur $F_{o}$; choisissant une uniformisante $\pi_{o}$ de $F_{o}$, on peut décrire l'ordre maximal $\mathcal{D}_{o}$ comme l'algèbre des séries formelles non commutatives en une indéterminée $\Pi_{o}$ soumises aux relations $\Pi_{o} a=$ $a^{q_{o}} \Pi_{o}\left(a \in \mathbb{F}_{q_{o}{ }^{d}}\right)$ et $\Pi_{o}^{d}=\pi_{o}$. On obtient ainsi un diagramme d'identifications :

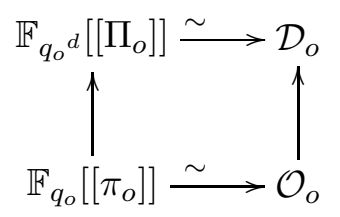

Proposition 2.7. - Supposons que o $\notin z(S)$ et donnons-nous $n \in \mathbb{N}^{*}$. La donnée d'une $n \cdot o$ structure de niveau sur $\left(\mathcal{E}_{i}, j, t\right)$ est équivalente à la donnée d'un isomorphisme $\mathcal{D}_{o}$-équivariant de $S$-schémas en $\mathcal{O}_{o}$-modules :

$$
\mathcal{D}_{o, n}^{\vee} \times S \stackrel{\sim}{\longrightarrow} G r_{o}(\mathcal{E})\left[\pi_{o}^{n}\right]
$$


où $\mathcal{D}_{o, n}^{\vee}$ est le $\mathbb{F}_{q_{o}}$-dual de $\mathcal{D}_{o, n}=\pi_{o}^{n} \mathcal{D}_{o} \backslash \mathcal{D}_{o}$ et $G r_{o}(\mathcal{E})\left[\pi_{o}^{n}\right]=\operatorname{Gr}\left(\mathcal{E}_{o, n}\right)$ désigne les point de $\pi_{o}^{n}$-torsion du groupe $\pi_{o}$-divisible $G r_{o}(\mathcal{E})$.

Dès que l'on choisit un diagramme d'identifications (1), c'est équivalent à la donnée d'un isomorphisme $\mathcal{D}_{o}$-équivariant :

$$
\mathcal{D}_{o} / \mathcal{D}_{o} \pi_{o}^{n} \times S \stackrel{\sim}{\longrightarrow} G r_{o}(\mathcal{E})\left[\pi_{o}^{n}\right]
$$

Démonstration. - Par définition, une $n \cdot o$-structure de niveau sur $\left(\mathcal{E}_{i}, j, t\right)$ consiste en la donnée d'un diagramme commutatif $\mathcal{D}_{o}$-équivariant :

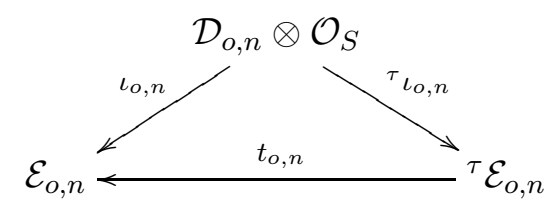

En d'autres termes, il s'agit d'un isomorphisme $\mathcal{D}_{o}$-équivariant entre les $\varphi$-faisceaux $\left(\mathcal{E}_{o, n}, t_{o}\right)$ et $\left(\mathcal{D}_{o, n} \otimes \mathcal{O}_{S}\right.$, Id). Par ailleurs, on voit facilement que $G r\left(\mathcal{D}_{o, n} \otimes \mathcal{O}_{S}\right)=S \times \mathcal{D}_{o, n}^{\vee}$. Le foncteur $G r$ étant pleinement fidèle (cf. proposition 2.3), une structure de niveau $n \cdot o$ est donc équivalente à un isomorphisme $\mathcal{D}_{o}$-équivariant de $S$-schémas en $\mathcal{O}_{o}$-modules :

$$
\mathcal{D}_{o, n}^{\vee} \times S \stackrel{\sim}{\longrightarrow} G r_{o}(\mathcal{E})\left[\pi_{o}^{n}\right] .
$$

Fixons maintenant un diagramme d'identifications (11). Notons $\operatorname{Tr}: D_{o} \rightarrow F_{o}$ la trace réduite; elle vérifie (cf. $[\mathbf{W}] \mathrm{X} \S 2$ prop. 5) :

$$
\operatorname{Tr}(x y) \in \mathcal{O}_{o} \forall x \in \mathcal{D}_{o} \Longleftrightarrow y \in \Pi_{o}^{1-d} \mathcal{D}_{o} .
$$

Désignant par Rés : $F_{o} / \mathcal{O}_{o} \rightarrow \mathbb{F}_{q_{o}}$ le morphisme « résidu », on vérifie alors facilement que l'application

$$
\begin{aligned}
& \left(\pi_{o}^{n} \mathcal{D}_{o} \backslash \mathcal{D}_{o}\right) \quad \otimes_{\mathbb{F}_{q_{o}}}\left(\mathcal{D}_{o} / \mathcal{D}_{o} \pi_{o}^{n}\right) \quad \longrightarrow \mathbb{F}_{q_{o}} \\
& a_{1} \otimes a_{2} \quad \longmapsto \quad \text { Rés } \circ \operatorname{Tr}\left(a_{2} \Pi_{o}^{1-d-n d} a_{1}\right)
\end{aligned}
$$

induit un isomorphisme de $\mathcal{D}_{o}$-modules à gauche

$$
\mathcal{D}_{o} / \mathcal{D}_{o} \pi_{o}^{n} \stackrel{\sim}{\longrightarrow}\left(\pi_{o}^{n} \mathcal{D}_{o} \backslash \mathcal{D}_{o}\right)^{\vee},
$$

d'où la deuxième assertion de la proposition.

2.3. Rappels sur le module de coordonnées des $\mathcal{O}$-modules formels. - Dans ce paragraphe, $\mathcal{O}$ est un anneau de valuation discrète complet d'égale caractéristique $p>0$ et de corps résiduel fini $\kappa=\mathbb{F}_{q^{\prime}}\left(q^{\prime}=p^{r}\right)$. On désigne par $K$ le corps des fractions de $\mathcal{O}$ et on fixe une uniformisante $\pi$ de $K$. D'autre part, soit $B$ une $\mathcal{O}$-algèbre dans laquelle l'image de $\pi$ est nilpotente. On note $i: \mathcal{O} \rightarrow B$ le morphisme structural et $\Gamma$ le morphisme $a \hat{\otimes}_{\kappa} b \in \mathcal{O} \hat{\otimes}_{\kappa} B \mapsto i(a) b$. Le morphisme

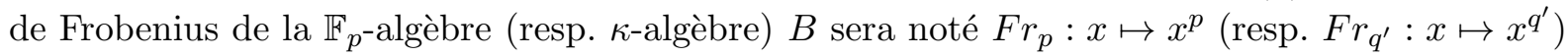
et $\tau_{p}$ (resp. $\tau_{q^{\prime}}$ ) désigne l'isogénie de Frobenius $\mathbb{G}_{a, B} \rightarrow F r_{p, *} \mathbb{G}_{a, B}$ (resp. $\mathbb{G}_{a, B} \rightarrow F r_{q^{\prime}, *} \mathbb{G}_{a, B}$ ).

Définition 2.8. - La catégorie $\operatorname{Mod} \mathcal{C}(B)$ des modules de coordonnées sur $B$ est la souscatégorie pleine de celle des $\left(\mathcal{O} \hat{\otimes}_{\kappa} B\right)$-modules localement libres $M$ munis d'un Frobenius $F$ : $\left(\mathrm{Id} \hat{\otimes}_{\kappa} F r_{q^{\prime}}\right)^{*} M \rightarrow M$, formée des objets tels que :

1. Le $\left(\mathcal{O} \hat{\otimes}_{\kappa} B\right)$-module $M$ est localement libre de rang fini;

2. Il existe un $B$-module localement libre de type fini $\omega$ tel que Coker $F=\Gamma_{*}(\omega)$;

3. Il existe un entier $n$ tel que le morphisme $F^{n}:\left(\operatorname{Id} \hat{\otimes}_{\kappa} F r_{q^{\prime}}^{n}\right)^{*} M / \pi M \rightarrow M / \pi M$ est le morphisme nul (on dit que le Frobenius est topologiquement nilpotent). 
D'autre part, on rappelle qu'un $\mathcal{O}$-module formel est un groupe formel lisse $X$ sur $B$, muni d'une action de $\mathcal{O}$, telle que l'action induite sur l'espace tangent Lie $X$ coïncide avec celle provenant de la structure de $B$-module de Lie $X$.

Théorème 2.9. - (cf. $\mathbf{G e}$ Th. 2.2.6) Il existe une anti-équivalence, entre la catégorie des $\mathcal{O}$-modules formels $X$ sur $B$ localement libres de hauteur finie et la catégorie $\operatorname{Mod} \mathcal{C}(B)$, donnée par le foncteur « module de coordonnées » $M_{B}: X \mapsto(M, F)$.

Expliquons en quelques mots la construction : à un $\mathcal{O}$-module formel $X$ sur $B$, on associe le $B$-module $M_{X}=\operatorname{Hom}\left(X, \mathbb{G}_{a, B}\right)$ (homomorphismes de groupes formels), lequel est muni par fonctorialité d'une action de $\mathcal{O}$. La multiplication à gauche par $\tau_{p}$ sur $M_{X}$ est $F r_{p}$-semi-linéaire et induit donc un morphisme $F: F r_{p}^{*} M_{X} \rightarrow M_{X}$. Par ailleurs, l'application qui a tout $m \in M_{X}$ associe sa différentielle définit un isomorphisme de $B$-modules $D:$ Coker $F \stackrel{\sim}{\rightarrow}(\operatorname{Lie} X)^{\vee}$.

Le $\left(\mathcal{O} \otimes_{\mathbb{F}_{p}} B\right)$-module $M_{X}$ est muni de deux actions de $\kappa$. Celles-ci induisent une graduation $M_{X}=\bigoplus_{i \in \mathbb{Z} / r \mathbb{Z}} M_{X, i}$, où $M_{X, i}=\left\{m \in M_{X}:(\lambda \otimes 1) m=t\left(1 \otimes \lambda^{p^{i}}\right) m\right\}$. Le morphisme $F$ induit des morphismes $B$-linéaires $F_{p, i}: F r_{p}^{*} M_{X, i} \rightarrow M_{X, i+1}$. On note $F_{0}:\left(F r_{q^{\prime}}\right)^{*} M_{X, 0} \rightarrow M_{X, 0}$ le morphisme $F_{p, r-1} \circ F r_{p}^{*} F_{p, r-2} \circ \cdots \circ\left(F r_{p}^{r-1}\right)^{*} F_{p, 0}$. On vérifie alors que se donner $\left(M_{X}, F\right)$ est équivalent à se donner $\left(M_{X, 0}, F_{0}\right)$. Pour finir, le séparé complété $\mathcal{O} \hat{\otimes}_{\kappa} B$ de $\mathcal{O} \otimes_{\kappa} B$ pour la topologie $\pi \otimes 1$-adique agit encore sur $M_{X, 0}$, parce que $X$ est la limite de schémas en groupes finis locaux.

Définition 2.10. - Le foncteur module de coordonnées sur $B$ est le foncteur $M_{B}$ qui à un $\mathcal{O}$-module formel $X$ sur $B$ associe le $\mathcal{O} \hat{\otimes}_{\kappa} B$-module $M_{B}(X)=M_{X, 0} \otimes_{\left(\mathcal{O} \otimes_{\kappa} B\right)} \mathcal{O} \hat{\otimes}_{\kappa} B$ muni du Frobenius semi-linéaire $F_{0}$.

Par construction, le rang du $\left(\mathcal{O} \hat{\otimes}_{\kappa} B\right)$-module $M_{B}(X)$ est la $\mathcal{O}$-hauteur du $\mathcal{O}$-module formel $X$ et $\omega$ est un $B$-module localement libre de $\operatorname{rang} \operatorname{dim} X$. Rappelons également que la formation de $M_{B}$ est compatible au changement de base (cf. Ge Prop. 2.2.10). Enfin, donnons le foncteur quasi-inverse $G_{B}$ : pour toute $B$-algèbre $R$,

$$
G_{B}(M, F)(R)=\left\{g \in \operatorname{Hom}_{B}(M, R): g(F(m))=g(m)^{q^{\prime}} \forall m \in M\right\} .
$$

\section{4. $\mathcal{O}$-modules de Dieudonné et $\mathcal{O}$-modules divisibles. -}

Définition 2.11. - Un $\mathcal{O}$-module de Dieudonné de rang $d$ sur $B$ est un $\left(\mathcal{O} \hat{\otimes}_{\kappa} B\right)$-module $M$ localement libre de rang $d$, muni d'un morphisme de Frobenius $F:\left(\operatorname{Id}_{\mathcal{O}} \hat{\otimes}_{\kappa} F r_{q^{\prime}}\right)^{*} M \rightarrow M$ tel que Coker $F \simeq \Gamma_{*}(\omega)$, où $\omega$ est un $B$-module localement libre de type fini.

On notera $\operatorname{Mod} \mathcal{B}(B)$ la catégorie des $\mathcal{O}$-modules de Dieudonné de rang fini sur $B$. Ainsi la catégorie $\operatorname{Mod} \mathcal{C}(B)$ définie en 2.3 est la sous-catégorie de $\operatorname{Mod} \mathcal{B}(B)$ dont les objets sont les modules $M$ munis d'un Frobenius $F$ topologiquement nilpotent. Lorsque $B$ est une $\mathcal{O}$-algèbre locale artinienne, on a la :

Proposition 2.12. - Soit $(M, F)$ un $\mathcal{O}$-module de Dieudonné sur $B$. Il existe des $\mathcal{O}$-modules de Dieudonné sur $B,\left(M^{e t}, F^{e t}\right)$ et $\left(M^{c}, F^{c}\right)$, ainsi qu'une suite exacte :

$$
0 \longrightarrow\left(M^{e t}, F^{e t}\right) \longrightarrow(M, F) \longrightarrow\left(M^{c}, F^{c}\right) \longrightarrow 0
$$

tels que $F^{e t}:\left(\operatorname{Id}_{\mathcal{O}} \hat{\otimes}_{\kappa} F r_{q^{\prime}}\right)^{*} M^{e t} \rightarrow M^{\text {et }}$ est bijectif et $F^{c}:\left(\operatorname{Id}_{\mathcal{O}} \hat{\otimes}_{\kappa} F r_{q^{\prime}}\right)^{*} M^{c} \rightarrow M^{c}$ est topologiquement nilpotent. 
Une démonstration, pour $B$ un corps, est donnée dans [Lau (proposition 2.4.6); le cas général s'y ramène facilement (voir $[$ Boy $]$ Lemme 6.2.3).

Définition 2.13. - Un $\mathcal{O}$-module divisible sur une $\mathcal{O}$-algèbre $B$ dans laquelle l'image de $\pi$ est nilpotente est un Spec $B$-faisceau $G$ en $\mathcal{O}$-modules, pour la topologie f.p.p.f., tel que la multiplication par $\pi$ est surjective et tel que, notant $G_{n}:=\operatorname{Ker}\left(\pi^{n}\right)$ pour tout entier $n \geq 1$, le $\mathcal{O}$-module $G_{1}$ est représentable par un Spec $B$-schéma en groupes fini localement libre et l'on a $G=\underset{n}{\lim } G_{n}$

Dans le cas où $B$ est artinienne, un $\mathcal{O}$-module divisible $G$ sur $B$ se décompose canoniquement :

$$
0 \longrightarrow G^{c} \longrightarrow G \longrightarrow G^{e t} \longrightarrow 0,
$$

où $G^{c}$ désigne la partie connexe et $G^{e t}$ le quotient étale maximal de $G$. On peut montrer que $G^{c}$ est en fait un $\mathcal{O}$-module formel (même démonstration que celle donnée par Messing pour les groupes $p$-divisibles; voir $[\mathbf{M e}]$ ).

Proposition 2.14. - Soit B une $\mathcal{O}$-algèbre locale artinienne; le foncteur $M_{B}$ induit une antiéquivalence, de la catégorie des $\mathcal{O}$-modules divisibles sur $B$ dans la catégorie $\operatorname{Mod} \mathcal{B}(B)$, qui prolonge l'équivalence du théorème 2.9 et respecte les décompositions canoniques de $(M, F)=$ $M_{B}(G)$ et $G=G_{B}(M, F)$ en leurs parties étales et connexes.

Une preuve assez brève est donnée dans Boy (Prop. 6.2.2). En fait, le résultat est sans doute vrai pour toute $\mathcal{O}$-algèbre $B$ dans laquelle l'image de $\pi$ est nilpotente, bien qu'aucune preuve n'ait été écrite à ce jour. Par ailleurs, la démonstration du théorème de Čerednik-Drinfeld (ou plutôt de son analogue) n'utilise que la version « modules formels - modules de coordonnées » de cette proposition (i.e. le théorème 2.9). En effet, on verra que le module divisible associé à un $\mathcal{D}$-faisceau elliptique « spécial » est un module formel.

2.5. $\mathcal{D}_{o}$-module de Dieudonné associé à un $\mathcal{D}$-faisceau elliptique. - Soit $o$ une place de $X \backslash\{\infty\}$ et $r$ le degré de $\kappa(o)$ sur $\mathbb{F}_{q}$; donnons-nous un $\mathcal{D}$-faisceau elliptique $\left(\mathcal{E}_{i}, j, t\right)$ défini sur une $\mathcal{O}_{o}$-algèbre locale artinienne $B$ (de morphisme structural le zéro $z: \mathcal{O}_{o} \rightarrow B$ de $\left(\mathcal{E}_{i}, j, t\right)$ ). On note toujours $F r_{q}$ (resp. $F r_{q_{o}}$ ) le Frobenius arithmétique de la $\mathbb{F}_{q}$-algèbre (resp. $\kappa(o)$-algèbre) $B$ et désigne par $\Gamma_{z}$ (resp. $\widetilde{\Gamma}_{z}$ ) le morphisme $\mathcal{O}_{o} \otimes_{\mathbb{F}_{q}} B \ni a \otimes b \mapsto z(a) . b \in B\left(\operatorname{resp} . \mathcal{O}_{o} \hat{\otimes}_{\kappa(o)} B \ni\right.$ $a \hat{\otimes} b \mapsto z(a) . b \in B)$.

Puisque $o$ et $\infty$ sont distincts, le $\mathcal{O}_{o} \otimes_{\mathbb{F}_{q}} B$-module $\mathcal{E}_{i} \otimes \mathcal{O}_{o}$ localement libre de rang $d^{2}$ est, via le morphisme $j$, indépendant de l'indice $i$; on le note $\mathcal{E}_{o}$. Il est naturellement muni d'un Frobenius $t_{o}:\left(\operatorname{Id}_{\mathcal{O}_{o}} \otimes_{\mathbb{F}_{q}} F r_{q}\right)^{*} \mathcal{E}_{o} \rightarrow \mathcal{E}_{o}$ issu du morphisme $t$.

De manière tout à fait similaire à la situation du paragraphe 2.3 , la double action de $\kappa(o)$ fait de $\mathcal{E}_{o}$ un $\mathcal{O}_{o} \otimes_{\mathbb{F}_{q}} B$-module gradué. On pose $M=\mathcal{E}_{o, 0}$ la composante de degré 0 et $F$ : $\left(\operatorname{Id}_{F_{o}} \otimes_{\kappa(o)} F r_{q_{o}}\right)^{*} M \rightarrow M$ le morphisme induit par $t_{o}$.

Il est alors aisé de vérifier que le $\mathcal{O}_{o} \hat{\otimes}_{\kappa(o)} B$-module $M_{o}=M \otimes_{\left(\mathcal{O}_{o} \otimes_{\kappa(o)} B\right)}\left(\mathcal{O}_{o} \hat{\otimes}_{\kappa(o)} B\right)$ muni du Frobenius $F_{o}$ déduit de $F$ appartient à la catégorie $\operatorname{Mod} \mathcal{B}(B)$ dont les objets sont les $\mathcal{O}_{o}$-modules de Dieudonné. Notons que $M_{o}$ est localement libre de rang $d^{2}$ et que Coker $F_{o}$ est l'image directe d'un $B$-module localement libre de rang $d$ par le morphisme $\widetilde{\Gamma}_{z}$.

Définition 2.15. - Soit $\left(\mathcal{E}_{i}, j, t\right)$ un $\mathcal{D}$-faisceau elliptique sur une $\mathcal{O}_{o}$-algèbre artinienne $B$.

Le $\mathcal{O}_{o}$-module de Dieudonné sur $B$ associé à $\left(\mathcal{E}_{i}, j, t\right)$ est le couple $\left(M_{o}, F_{o}\right)$ où

$$
M_{o}=\mathcal{E}_{o, 0} \otimes_{\left(\mathcal{O}_{o} \otimes_{\kappa(o)} B\right)}\left(\mathcal{O}_{o} \hat{\otimes}_{\kappa(o)} B\right)
$$


et $F_{o}:\left(\operatorname{Id}_{\mathcal{O}_{o}} \hat{\otimes}_{\kappa(o)} F r_{q_{o}}\right)^{*} M_{o} \rightarrow M_{o}$ est le Frobenius induit par $t_{o}$.

La proposition suivante établit le lien entre les différentes notions rencontrées au cours de cette section. En fait, c'est un résultat immédiat, lorsque l'on compare la construction du schéma $G r_{o}$ avec la définition du foncteur $G_{B}$.

Proposition 2.16. - Le $\mathcal{O}_{o}$-module de Dieudonné $\left(M_{o}, F_{o}\right)$ associé à $\left(\mathcal{E}_{i}, j, t\right)$ et le $\mathcal{O}_{o}$-module divisible $G_{o}(\mathcal{E})$ sur $B$ associé à $\left(\mathcal{E}_{i}, j, t\right)$ (cf. définition 2.4) se correspondent par l'anti-équivalence de catégorie décrite au paragraphe 2.4.

Remarque. - De plus, $M_{o}$ est muni d'une action à droite de $\mathcal{D}_{o}=\mathcal{D} \otimes_{\mathcal{O}_{X}} \mathcal{O}_{o}$ qui prolonge celle de $\mathcal{O}_{o}$ et commute avec $F_{o}$. On dit alors que $\left(M_{o}, F_{o}\right)$ est un $\mathcal{D}_{o^{-}}$-module de Dieudonné.

\section{La condition « spéciale "}

La condition « spéciale » porte directement sur le $\mathcal{O}_{o}$-module divisible $G r_{o}(\mathcal{E})$. Avant de l'énoncer, il nous faut procéder à quelques rappels sur les $\mathcal{O}_{D}$-modules formels spéciaux. A ce propos, on citera les principaux résultats et renvoie le lecteur à [Dr5] ou [Ge I pour les détails.

3.1. $\mathcal{O}_{D}$-modules formels spéciaux. - Poursuivant avec les notations de 2.3, on désigne par $\mathcal{O}_{d}$ l'anneau des entiers de l'extension non-ramifiée de degré $d, K_{d}$, de $K$ et par $\mathcal{O}_{D}$ l'ordre maximal de l'algèbre à division centrale simple $D$ d'invariant $1 / d$ sur $K$.

Définition 3.1. - Un $\mathcal{O}_{D}$-module formel est un groupe formel lisse $X$ sur $B$ muni d'une action de $\mathcal{O}_{D}$ telle que le groupe formel avec action de $\mathcal{O}$ sous-jacent soit un $\mathcal{O}$-module formel. Il est dit spécial lorsque l'action de $\mathcal{O}_{d} \subset \mathcal{O}_{D}$ induite sur l'espace tangent Lie $X$ fait de Lie $X$ un $\mathcal{O}_{d} \otimes_{\mathcal{O}} B$-module inversible.

Soit $X$ un $\mathcal{O}_{D}$-module formel de hauteur finie sur $B$. On dit que le module de coordonnées $(M, F)=M_{B}(X)$, muni par fonctorialité d'une action de $\mathcal{O}_{D}$, est un $\mathcal{O}_{D}$-module de coordonnées. Voyons comment se traduit la spécialité.

Fixons un diagramme d'identifications

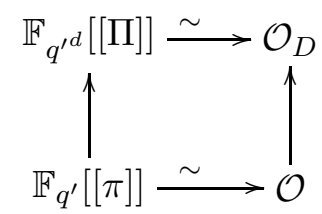

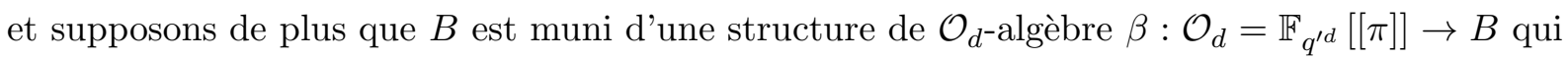
prolonge sa structure de $\mathcal{O}$-algèbre. Le module de coordonnées $M$ est alors muni de deux actions de $\mathbb{F}_{q^{\prime d}}$, obtenues en considérant les plongements $\mathbb{F}_{q^{\prime d}} \subset \mathcal{O}_{D}$ et $\mathbb{F}_{q^{\prime d}} \subset B$. Celles-ci induisent une graduation (on redécompose!) $M=\bigoplus_{i \in \mathbb{Z} / d \mathbb{Z}} M_{i}$, où l'on pose $M_{i}=\operatorname{Ker}\left(a \hat{\otimes} 1-1 \hat{\otimes} a^{q^{i i}}\right)$, pour $a$ quelconque engendrant $\mathbb{F}_{q^{\prime d}}$ sur $\mathbb{F}_{q^{\prime}}$. L'endomorphisme $\Pi \hat{\otimes} 1$ est de degré un pour cette graduation et vérifie $(\Pi \hat{\otimes} 1)^{d}=\pi \hat{\otimes} 1$. Il injecte donc $M_{i}$ dans $M_{i+1}$ et les $M_{i}$ sont des $\mathcal{O} \hat{\otimes}_{\mathbb{F}_{q^{\prime}}} B$ modules localement libres tous de même rang. En particulier, la hauteur de $X$ est multiple de $d$. De même, le morphisme $F$ définit une injection $F_{i}:\left(\operatorname{Id}_{\mathcal{O}} \hat{\otimes}_{\mathbb{F}_{q^{\prime}}} F r_{q^{\prime}}\right)^{*} M_{i} \rightarrow M_{i+1}$. Le conoyau Coker $F=\Gamma_{*}(\omega)$ se décompose en somme directe de quotients Coker $F_{i}=\Gamma_{*}\left(\omega_{i}\right)$.

Proposition 3.2. - Avec les notations précédentes, $M$ est spécial si et seulement si les $\omega_{i}$ sont des $B$-modules localement libres tous de même rang égal à un. 
Démonstration. - En effet, la décomposition précédente correspond à une décomposition du dual de l'espace tangent $(\operatorname{Lie} X)^{\vee}=\bigoplus_{i}(\operatorname{Lie} X)_{i}^{\vee}$, où :

$$
(\operatorname{Lie} X)_{i}^{\vee}=\left\{c \in(\operatorname{Lie} X)^{\vee}:(a \hat{\otimes} 1) c=\left(1 \hat{\otimes} a^{q^{i}}\right) c \quad \forall a \in \mathbb{F}_{q^{\prime}}\right\} .
$$

Désignant par $\tau$ le pull-back par $\operatorname{Id}_{\mathcal{O}_{d}} \hat{\otimes}_{\mathbb{F}_{q^{\prime}}} F r_{q^{\prime}}$, on dit que $i \in \mathbb{Z} / d \mathbb{Z}$ est critique si l'application $\bar{\Pi}_{i}: M_{i} / F\left({ }^{\tau} M_{i-1}\right) \rightarrow M_{i+1} / F\left({ }^{\tau} M_{i}\right)$ à travers laquelle se factorise $\Pi \hat{\otimes} 1$ est l'application nulle. Autrement dit, l'indice $i$ est critique si $(\Pi \hat{\otimes} 1)\left(M_{i}\right) \subset F\left({ }^{\tau} M_{i}\right)$.

Lemme 3.3. - Si B est intègre alors il existe au moins un indice critique.

Démonstration. - Cela résulte de l'observation suivante : l'application $(\Pi \hat{\otimes} 1)^{d}=\pi \hat{\otimes} 1$ induit l'application nulle sur l'espace tangent Lie $X$ mais se factorise comme une composée d'applications $\bar{\Pi}_{i}$ entre $B$-modules localement libres de rang un. Nécessairement l'un des $\bar{\Pi}_{i}$ est nul.

Nous pouvons maintenant démontrer le résultat fondamental suivant :

Théorème 3.4. - (cf. [Dr4] §2) Tous les $\mathcal{O}_{D}$-modules formels spéciaux $X$ de hauteur $d^{2}$ sur une extension algébriquement close de $\mathbb{F}_{q^{\prime d}}$ sont $\left(\mathcal{O}_{D}\right.$-linéairement) isogènes; de plus, on a:

$$
\operatorname{End}_{D}^{0} X=\operatorname{End}_{\mathcal{O}_{D}} X \otimes K \simeq \mathrm{M}_{d}(K) .
$$

Démonstration. - En vertu de Ge prop. 2.2.11, la classe d'isogénie du $\mathcal{O}_{D^{-}}$-module formel $X$ est déterminée par le $K$-module de Dieudonné $\left(M \otimes_{\mathcal{O}} K, F \otimes_{\mathcal{O}} \operatorname{Id}_{K}\right)$ sur $B=\overline{\mathbb{F}}_{q^{\prime}}$ muni de l'action de $D$ induite (dans sa classe d'isomorphisme). Or les injections $\Pi \hat{\otimes} 1: M_{i} \hookrightarrow M_{i+1}$ induisent par tensorisation avec $K$ des isomorphismes $\Pi_{i, K}: M_{i, K} \stackrel{\sim}{\rightarrow} M_{i+1, K}$ entre $K \hat{\otimes}_{\mathbb{F}_{q^{\prime}}} B$ modules libres de rang $d$ (on note $M_{i, K}=M_{i} \otimes_{\mathcal{O}} K$ ). Ces isomorphismes sont compatibles avec le Frobenius $F_{K}=F \otimes_{\mathcal{O}} \operatorname{Id}_{K}$, au sens où $F_{i+1, K} \circ\left(\operatorname{Id}_{K} \hat{\otimes}_{\mathbb{F}_{q^{\prime}}} F r_{q^{\prime}}\right)^{*} \Pi_{i, K}=\Pi_{i+1, K} \circ F_{i, K}$. Le module de Dieudonné $\left(M \otimes_{\mathcal{O}} K, F \otimes_{\mathcal{O}} \operatorname{Id}_{K}\right)$ est donc déterminé par la donnée d'un des modules de Dieudonné $\left(M_{i, K}, \Pi_{i, K}^{-1} \circ F_{i, K}\right)$.

Choisissons un indice critique $i$ et raisonnons sur le réseau $M_{i}$ de $M_{i, K}$. Le fait que $i$ est critique implique que $M_{i}$ est stable par $\Pi_{i, K}^{-1} \circ F_{i, K}$ et que l'application $\Pi_{i, K}^{-1} \circ F_{i, K}:\left(\operatorname{Id}_{K} \hat{\otimes}_{\mathbb{F}_{q^{\prime}}} F r_{q^{\prime}}\right)^{*} M_{i} \rightarrow$ $M_{i}$ est bijective. En effet, l'inclusion $(\Pi \hat{\otimes} 1)\left(M_{i}\right) \subset F\left({ }^{\tau} M_{i}\right)$ est une égalité car les réseaux $(\Pi \hat{\otimes} 1)\left(M_{i}\right)$ et $F\left({ }^{\tau} M_{i}\right)$ ont même indice dans $M_{i+1}$. On prouve cette dernière assertion comme suit : puisque $M$ est spécial, les $B$-modules $M_{i+1} / F\left({ }^{\tau} M_{i}\right)$ sont libres de rang un pour tout $i$; considérant alors le diagramme commutatif

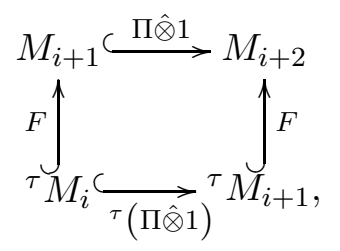

on voit que le rang du $B$-module libre $M_{i+1} /(\Pi \hat{\otimes} 1) M_{i}$ est indépendant de $i$. Par ailleurs, $M /(\pi \hat{\otimes} 1) M$ est un $B$-module libre de rang $d^{2}$, donc $M /(\Pi \hat{\otimes} 1) M$ est libre de rang $d$; il se décompose en une somme directe $M /(\Pi \hat{\otimes} 1) M=\bigoplus_{i=1}^{d} M_{i+1} /(\Pi \hat{\otimes} 1) M_{i}$. Ainsi $M_{i+1} /(\Pi \hat{\otimes} 1) M_{i}$ est de rang un, donc de même rang que $M_{i+1} / F\left({ }^{\tau} M_{i}\right)$, pour tout $i$.

Comme $B$ est algébriquement clos, il existe d'après [Zi2] VI $\S 4$ Lemma 6.25 une base $e_{1}, \ldots, e_{d}$ de $M_{i}$ sur $\mathcal{O} \hat{\otimes}_{\mathbb{F}_{q^{\prime}}} B$ telle que $\Pi_{i, K}^{-1} \circ F_{i, K}\left(e_{j}\right)=e_{j}$ pour tout $1 \leq j \leq d$. Ceci détermine $\left(M_{i, K}, \Pi_{i, K}^{-1} \circ\right.$ $F_{i, K}$ ) (à isomorphisme près). 
Enfin, les correspondances $X \mapsto\left(M_{X} \otimes_{\mathcal{O}} K, F_{X} \otimes_{\mathcal{O}} \operatorname{Id}_{K}\right) \mapsto\left(M_{i, K}, \Pi_{i, K}^{-1} \circ F_{i, K}\right)$ induisent des isomorphismes :

$$
\operatorname{End}_{\mathcal{O}_{D}} X \otimes K=\operatorname{End}_{D}\left(M_{X} \otimes_{\mathcal{O}} K, F_{X} \otimes_{\mathcal{O}} \operatorname{Id}_{K}\right)=\operatorname{End}_{K}\left(M_{i, K}, \Pi_{i, K}^{-1} \circ F_{i, K}\right) .
$$

$\operatorname{Or}_{\operatorname{End}_{K}}\left(M_{i, K}, \Pi_{i, K}^{-1} \circ F_{i, K}\right) \simeq \mathrm{M}_{d}(K)$, puisqu'un endomorphisme $K$-linéaire de $M_{i, K}$ commute

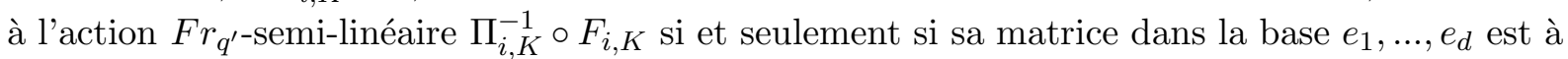
coefficients dans $\left(K \otimes_{\mathbb{F}_{q^{\prime}}} B\right)^{F r_{q^{\prime}}}=K$.

Le lecteur trouvera dans Ge I 4.2 une description « du $» \mathcal{O}_{D}$-module formel spécial $\Phi$ de hauteur $d^{2}$ sur $\overline{\mathbb{F}}_{q^{\prime}}$ (à isogénie près).

3.2. Enoncé de la condition spéciale - - On rappelle que $o$ est une place de $F$ telle que $D_{o}$ est un corps gauche d'invariant $1 / d$ sur $F_{o}$. Nous notons $\mathcal{O}_{o}^{(d)} \subset \mathcal{D}_{o}$ l'anneau des entiers de l'extension non ramifiée de degré $d$ de $F_{o}$ (plongement bien défini à conjugaison près). Etant donné un $\mathcal{D}$-faisceau elliptique $\mathcal{E}=\left(\mathcal{E}_{i}, j, t\right)$ sur un $\mathbb{F}_{q}$-schéma $S$, nous disposons du groupe $\pi_{o}$-divisible $G r_{o}(\mathcal{E})$ défini en 2.2 , La condition spéciale porte directement sur ce groupe.

Définition 3.5. - On dit que $\left(\mathcal{E}_{i}, j, t\right)$ est spécial s'il vérifie la condition supplémentaire suivante :

(v) [Cond. spéciale] : Pour chaque point géométrique $s=\operatorname{Spec} \kappa(s)$ de caractéristique $o$ de $S$ (i.e. tel que $z(s)=o$ ), l'action de $\mathcal{O}_{o}^{(d)}$ sur Lie $\left(G r_{o}(\mathcal{E})_{s}\right)$ se décompose comme la somme des $d$ plongements $\mathcal{O}_{o}^{(d)} \otimes \kappa(o) \simeq \mathbb{F}_{q_{o}^{d}} \hookrightarrow \kappa(s)$.

Remarques. - (a) Tout d'abord, interprétons la condition spéciale en terme du $\mathcal{D}_{o}$-module divisible $G=G r_{o}(\mathcal{E})$. Soit $s=\operatorname{Spec} \kappa(s)$ un point géométrique de caractéristique $o$ de $S$; clairement, $G_{s}$ ne peut être étale, lorsque la condition sur Lie $\left(G_{s}\right)$ est satisfaite : dans ce cas $G_{s}^{e t}=\operatorname{Spec} \kappa(s) \times\left(F_{o} / \mathcal{O}_{o}\right)^{j}$, où $j$ est un entier positif strictement inférieur à $d^{2}$. Or l'algèbre $D_{o}$ agit sur $G_{s}^{e t}$. Un calcul direct des dimensions couplé au lemme suivant montre alors que $j=0$; autrement dit, $G r_{o}(\mathcal{E})_{s}$ est un $\mathcal{D}_{o}$-module formel sur $\operatorname{Spec} \kappa(s)$.

Lemme 3.6. - Soit $D$ une algèbre à division de centre $k$. Tout D-module $M$ (non nul) est isomorphe à une somme directe de copies de D. En particulier, la plus petite représentation (non triviale) de $D$ sur $k$ est donnée par $\rho: D \hookrightarrow$ End $D$, c'est-à dire $D$ agissant sur elle-même par multiplication à gauche.

Démonstration. - En effet, tout $D$-module est somme de $D$-modules simples, donc isomorphes à $D$ car $D$ est simple.

Finalement, dire que $\left(\mathcal{E}_{i}, j, t\right)$ est un $\mathcal{D}$-faisceau elliptique spécial sur $S$ équivaut à dire que le groupe formel $G r_{o}(\mathcal{E})$ associé est un $\mathcal{D}_{o}$-module formel spécial, au sens où $G r_{o}(\mathcal{E})_{s}$ est spécial pour tout point géométrique $s$ de $S$ qui provient de la caractéristique $o$.

(b) La condition spéciale est une condition au-dessus de $\operatorname{Spec} \mathcal{O}_{o}$. Soit $S$ un $\mathcal{O}_{o}$-schéma; quitte à effectuer un changement de base étale (ce qui ne change en rien la structure de l'algèbre de Lie du groupe $\pi_{o}$-divisible $G r_{o}(\mathcal{E})$ ), on peut toujours supposer que $S$ est en fait un schéma sur l'extension non ramifiée $\mathcal{O}_{o}^{(d)}$ de degré $d$ de $\mathcal{O}_{o}$. Notons $\sigma$ l'automorphisme de la $\mathcal{O}_{o}$-algèbre $\mathcal{O}_{o}^{(d)}$ induisant l'automorphisme de Frobenius $x \mapsto x^{q}$ sur le corps résiduel. Le $\mathcal{O}_{S}$-module Lie $G r_{o}(\mathcal{E})$ est alors muni d'une action de l'anneau $\mathcal{O}_{o}^{(d)} \otimes_{\mathcal{O}_{o}} \mathcal{O}_{o}^{(d)}$, isomorphe à $\bigoplus_{i=1}^{d} \mathcal{O}_{o}^{(d)}$. Cette action décompose Lie $G r_{o}(\mathcal{E})$ en la somme directe de $d$ modules projectifs $\bigoplus_{i=1}^{d} \operatorname{Lie}^{i}\left(G r_{o}(\mathcal{E})\right)$, de telle sorte que $\mathcal{O}_{o}^{(d)}$ opère sur la $i^{\mathrm{e}}$ composante par le composé du 
morphisme structural $\mathcal{O}_{o}^{(d)} \rightarrow \mathcal{O}_{S}$ avec la puissance $\sigma^{i}$ de l'automorphisme $\sigma$. La condition spéciale signifie que le rang de chacun de ces $d$ modules sur $\mathcal{O}_{S}$ est égal à 1 en chaque point géométrique de caractéristique $o$ de $S$.

Cela a encore un sens en fibre générique de $\operatorname{Spec} \mathcal{O}_{o}$ et la condition est alors automatiquement vérifiée. En effet, notant $\bar{F}_{o}$ une clôture séparable du corps des fractions $F_{o}$ de $\mathcal{O}_{o}$, l'action de $\mathcal{D}_{o}$ sur le $\mathcal{O}_{o}^{(d)} \otimes_{\mathcal{O}_{o}} \bar{F}_{o} \simeq \bar{F}_{o}^{d}$-module Lie $\left(G r_{o}(\mathcal{E})\right) \otimes \bar{F}_{o}$ libre de rang 1 induit une injection $\mathcal{D}_{o} \hookrightarrow \operatorname{End}\left(\bar{F}_{o}^{d}\right)=\mathrm{M}_{d}\left(\bar{F}_{o}\right)$. Or l'unique application $\mathcal{D}_{o} \otimes_{\mathcal{O}_{o}} \bar{F}_{o}=\mathrm{M}_{d}\left(\bar{F}_{o}\right) \hookrightarrow \mathrm{M}_{d}\left(\bar{F}_{o}\right)$ correspond à la représentation que l'on s'imagine, c'est-à-dire l'algèbre $\mathrm{M}_{d}\left(\bar{F}_{o}\right)$ opérant sur elle-même par multiplication à gauche; de plus, dans cette représentation, $\mathcal{O}_{o}^{(d)} \hookrightarrow \mathcal{D}_{o}$ agit via $\mathcal{O}_{o}^{(d)} \hookrightarrow \operatorname{End}\left(\mathcal{O}_{o}^{(d)}\right)=\mathrm{M}_{d}\left(\mathcal{O}_{o}\right)$. L'action de $\mathcal{D}_{o}$ sur Lie $\left(G r_{o}(\mathcal{E})\right) \otimes \bar{F}_{o}$ fait donc bien intervenir tous les $d$ plongements $\mathcal{O}_{o}^{(d)} \hookrightarrow \bar{F}_{o}$ (à travers l'isomorphisme canonique $\mathcal{O}_{o}^{(d)} \otimes_{\mathcal{O}_{o}} \bar{F}_{o} \simeq \bar{F}_{o}^{d}$ ).

Parce que le rang d'un $\mathcal{O}_{S}$-module projectif est localement constant, on voit que la condition spéciale est une condition à la fois ouverte et fermée. Ainsi elle est satisfaite dès qu'elle l'est en un seul point géométrique de chaque composante connexe du schéma $S$. En particulier, la condition est automatiquement vérifiée par un $\operatorname{Spec} \mathcal{O}_{o}$-schéma plat (car elle est satisfaite en fibre générique).

\section{Analogue du Théorème de Serre et Tate}

Soient $S$ le spectre d'un anneau $R$ local artinien et $\bar{S} \subset S$ le sous-schéma fermé défini par un idéal $\mathfrak{m}$ de carré nul. Soit $\overline{\mathcal{E}}=\left(\overline{\mathcal{E}}_{i}, \bar{j}, \bar{t}\right)$ un $\mathcal{D}$-faisceau elliptique défini sur $\bar{R}=R / \mathfrak{m}$, de caractéristique $\bar{z}: \bar{S} \rightarrow \operatorname{Spec}\left(\mathcal{O}_{o}\right)$. On ne fixe pas le relèvement $z: S \rightarrow \operatorname{Spec}\left(\mathcal{O}_{o}\right)$ de $\bar{z}$. Notons $D e f_{R}(\overline{\mathcal{E}})$ l'ensemble des (classes d'isomorphie de) déformations de $\overline{\mathcal{E}}$ définies sur $R$. D'autre part, soit $\bar{M}=\left(\bar{M}_{o}, \bar{F}_{o}\right)$ le $\mathcal{O}_{o}$-module de Dieudonné associé à $\overline{\mathcal{E}}$. On désigne par $\operatorname{Def} f_{R}(\bar{M})$ l'ensemble des (classes d'isomorphie de) déformations de $\bar{M}$ définies sur $R$. Dans ce qui suit, dès que nous parlerons de $\mathcal{D}$-faisceau elliptique spécial, il sera implicitement sous-entendu que l'algèbre $D$ est, en la place $o$, un corps gauche $D_{o}$ d'invariant $1 / d$.

Théorème 4.1. - L'application qui à un $\mathcal{D}$-faisceau elliptique associe son $\mathcal{D}_{o}$-module de Dieudonné (cf. définition (2.15) induit une bijection $\operatorname{De} f_{R}(\overline{\mathcal{E}}) \rightarrow D e f_{R}(\bar{M})$. De plus, cette application préserve la spécialité.

Ainsi, en vertu de l'anti-équivalence de catégories entre modules de coordonnées et modules formels (cf. proposition 2.9), déformer un $\mathcal{D}$-faisceau elliptique spécial revient à déformer le $\mathcal{D}_{o^{-}}$ module formel $G r_{o}(\mathcal{E})$ sous-jacent. Cet énoncé constitue donc l'analogue pour les $\mathcal{D}$-faisceaux elliptiques du théorème de Serre et Tate dans le cadre des variétés abéliennes. La preuve se fait en calculant plus précisément les espaces de déformations des deux types d'objets en question. Signalons que l'étude des déformations (relatives au-dessus de $X$, c'est-à-dire à zéro $z$ fixé, ce que nous ne supposons pas ici) des $\mathcal{D}$-faisceaux elliptiques a déjà été esquissée dans [L-R-S (remark 4.10) en tant que méthode alternative à la démonstration de la lissité du schéma de modules des $\mathcal{D}$-faisceaux elliptiques. Ces idées ont été mises en forme plus tard par Boyer dans sa thèse (voir $\mathbf{B o y})$.

4.1. Déformation des $\mathcal{D}$-faisceaux elliptiques. - On poursuit avec les notations précédentes; notant $\Gamma_{\bar{z}}$ le graphe du morphisme $\bar{z}$, on a la suite exacte suivante :

$$
0 \longrightarrow \tau \overline{\mathcal{E}}_{0} \stackrel{\bar{t}}{\longrightarrow} \overline{\mathcal{E}}_{1} \longrightarrow\left(\Gamma_{\bar{z}}\right)_{*} \overline{\mathcal{B}} \longrightarrow 0
$$


où $\overline{\mathcal{B}}$ est un $\bar{R}$-module libre de rang $d$.

Proposition 4.2. - Il n'y a pas d'obstruction à relever le $\mathcal{D}$-faisceau elliptique $\overline{\mathcal{E}}$. L'ensemble des relèvements est un torseur sous le groupe

$$
\operatorname{Ext}_{\mathcal{D} \unrhd \mathcal{O}_{\bar{S}}}^{1}\left(\operatorname{Coker} \bar{t}, \overline{\mathcal{E}}_{1} \otimes_{\bar{R}} \mathfrak{m}\right) \simeq \operatorname{Ext}_{\mathcal{D}_{o} \bowtie \mathcal{O}_{\bar{S}}}^{1}\left(\operatorname{Coker} \bar{t}_{o}, \overline{\mathcal{E}}_{1, o} \otimes_{\bar{R}} \mathfrak{m}\right) .
$$

Démonstration. - Le même raisonnement que Boy $§ 5.1$ montre qu'il n'y a pas d'obstruction à relever la suite exacte (2) et que l'ensemble des relèvements est un torseur sous le groupe $\operatorname{Ext}_{\mathcal{D} \bowtie \mathcal{O}_{\bar{S}}}^{1}\left(\right.$ Coker $\left.\bar{t}, \overline{\mathcal{E}}_{1} \otimes_{\bar{R}} \mathfrak{m}\right)$, lequel est encore isomorphe à $\operatorname{Ext}_{\mathcal{D}_{o} \bowtie \mathcal{O}_{\bar{S}}}^{1}\left(\right.$ Coker $\left.\bar{t}_{o}, \overline{\mathcal{E}}_{1, o} \otimes_{\bar{R}} \mathfrak{m}\right)$. Ensuite, on vérifie comme dans loc. cit. que le relèvement $\mathcal{E}=\left(\mathcal{E}_{i}, j, t\right)$ de $\overline{\mathcal{E}}$ est entièrement déterminé par $t:{ }^{\tau} \mathcal{E}_{0} \rightarrow \mathcal{E}_{1}$. Noter que le conoyau Coker $t$ est l'image directe $\left(\Gamma_{z}\right)_{*} \mathcal{B}$ d'un $R$-module $\mathcal{B}$ localement libre de rang $d$ par la section $\Gamma_{z}$, graphe $d$ 'un certain morphisme $z$ relevant $\bar{z}$.

4.2. Déformation des $\mathcal{D}_{o}$-modules de Dieudonné. — Soit $\bar{M}=\left(\bar{M}_{o}, \bar{F}_{o}\right)$ un $\mathcal{D}_{o}$-module de Dieudonné de rang $d^{2}$ sur $\bar{R}$.

Proposition 4.3. - Il n'y a pas d'obstruction à relever le $\mathcal{D}_{o}$-module de Dieudonné $\bar{M}$. L'ensemble des relèvements est un torseur sous le groupe

$$
\operatorname{Ext}_{\mathcal{D}_{o} \hat{\otimes}_{\kappa(o)} \bar{R}}^{1}\left(\text { Coker } \bar{F}_{o}, \bar{M}_{o} \otimes_{\bar{R}} \mathfrak{m}\right) .
$$

De plus, si $\bar{M}$ est en fait un module de coordonnées spécial, alors il en est de même des relèvements.

Démonstration. - Relever le $\mathcal{D}_{o}$-module de Dieudonné $\bar{M}$ revient à relever la suite exacte

$$
0 \longrightarrow \tau \bar{M}_{o} \stackrel{\bar{F}_{o}}{\longrightarrow} \bar{M}_{o} \longrightarrow\left(\Gamma_{\bar{z}}\right)_{*} \bar{\omega} \longrightarrow 0
$$

où $\bar{\omega}$ désigne un $\bar{R}$-module libre de rang fini et $\Gamma_{\bar{z}}$ le morphisme $\mathcal{O}_{o} \hat{\otimes}_{\kappa(o)} \bar{R} \ni a \hat{\otimes} b \mapsto \bar{z}(a) b \in \bar{R}$. La preuve des deux premières assertions est de ce fait similaire à celle de la proposition 4.2.

Démontrons le dernier point : supposant que $\bar{M}$ est un module de coordonnées spécial, soit $M=\left(M_{o}, F_{o}\right)$ un relèvement de $\bar{M}$ vu comme $\mathcal{D}_{o}$-module de Dieudonné. Vérifions que le Frobenius $F_{o}$ est topologiquement nilpotent : si $n$ est un entier tel que $\bar{F}_{o}^{n}=0$, alors l'application $F_{o}^{n}$ est à valeurs dans $\left(\bar{M}_{o} / \pi_{o} \bar{M}_{o}\right) \otimes_{\bar{R}} \mathfrak{m} \subset M_{o} / \pi_{o} M_{o}$; puisque l'idéal $\mathfrak{m}$ est de carré nul, il vient $F_{o}^{2 n}=0$. Il reste à montrer que le module de coordonnées $M$ vérifie la condition spéciale. Pour cela, on peut toujours supposer, quitte à effectuer un changement de base étale, que $\bar{R}$ est une algèbre sur l'extension non ramifiée $\mathcal{O}_{d}$ de degré $d$ de $\mathcal{O}$. Le module de coordonnées $M$ se décompose alors suivant la double action de $\mathbb{F}_{q_{o}^{d}}$; écrivant Coker $\bar{F}_{o}=\left(\Gamma_{\bar{z}}\right)_{*}(\bar{\omega})=\bigoplus_{i \in \mathbb{Z} / d \mathbb{Z}}\left(\Gamma_{\bar{z}}\right)_{*}\left(\bar{\omega}_{i}\right)$, où les $\bar{\omega}_{i}$ sont des $\bar{R}$-modules libres de rang un (voir proposition 3.2), cette décomposition se relève en Coker $F_{o}=\bigoplus_{i \in \mathbb{Z} / d \mathbb{Z}}\left(\Gamma_{z}\right)_{*}\left(\omega_{i}\right)$ (pour un certain morphisme $z: S \rightarrow \operatorname{Spec}\left(\mathcal{O}_{o}\right)$ relevant $\bar{z}$ ), et les $R$-modules $\omega_{i}$ sont libres de rang un également par le lemme de Nakayama. Le lecteur notera que ces dernières vérifications reviennent à remarquer que la condition spéciale pour les modules de coordonnées (ou de manière équivalente les modules formels) est vérifiée dès qu'elle l'est sur la fibre spéciale, qui reste inchangée par déformation.

4.3. Preuve du théorème. - Le théorème 4.1 est un corollaire immédiat des propositions 4.2 et 4.3 . Noter que la spécialité est clairement conservée, puisque la condition spéciale porte justement sur la fibre spéciale qui reste inchangée par déformation. 


\section{Unicité de la classe d'isogénie}

Soit $\overline{\kappa(o)}$ une clôture algébrique du corps résiduel $\kappa(o)$; le but de cette section est de montrer que tous les $\mathcal{D}$-faisceaux elliptiques spéciaux sur $\overline{\kappa(o)}$ sont « isogènes », au sens de la définition 5.1 .

5.1. Fibre générique d'un $\mathcal{D}$-faisceau elliptique. - A un $\mathcal{D}$-faisceau elliptique $\left(\mathcal{E}_{i}, j, t\right)$ de caractéristique $o$ sur $\overline{\kappa(o)}$, c'est-à-dire dont le zéro est le morphisme $\operatorname{Spec} \overline{\kappa(o)} \rightarrow \operatorname{Spec} \kappa(o) \hookrightarrow X$, on associe un $\varphi$-espace $(V, \varphi)$ (au sens de [L-R-S A.1) et un homomorphisme de $F$-algèbre $\lambda: D^{o p p} \rightarrow \operatorname{End}(V, \varphi)$ de la façon suivante. Soit $V$ la fibre générique de $\mathcal{E}_{0}$; via le morphisme $j$ on peut identifier $V$ à la fibre générique de $\mathcal{E}_{i}$ pour tout $i$. L'application $t$ induit alors une application bijective $F \otimes_{\mathbb{F}_{q}} F r_{q}$-semi-linéaire bijective $\varphi: V \rightarrow V$ et $(V, \varphi)$ est un $\varphi$-espace sur $\overline{\kappa(o)}$. De plus, l'action (à droite) de $D$ sur $V$ commute avec $\varphi$ et fournit le morphisme $\lambda$.

Définition 5.1. - Le triplet $(V, \varphi, \lambda)$ est appelé la fibre générique du $\mathcal{D}$-faisceau elliptique $\left(\mathcal{E}_{i}, j, t\right)$. Deux faisceaux elliptiques sont dits isogènes si leurs fibres génériques sont isomorphes. B.1)

Si $x$ est une place de $F$, on considère le $F_{x}$-module de Dieudonné sur $\overline{\kappa(o)}$ (au sens de [L-R-S

$$
\left(V_{x}, \varphi_{x}\right)=\left(F_{x} \hat{\otimes}_{F} V, \operatorname{Id}_{F_{x}} \hat{\otimes} \varphi\right),
$$

muni du morphisme de $F_{x}$-algèbres $\lambda_{x}: D^{\text {opp }} \rightarrow$ End $\left(V_{x}, \varphi_{x}\right)$. On pose

$$
M_{x}=H^{0}\left(\operatorname{Spec}\left(\mathcal{O}_{x} \hat{\otimes} \overline{\kappa(o)}\right), \mathcal{E}_{0}\right),
$$

qui est un $\mathcal{D}_{x}$-réseau stable sous $\lambda_{x}\left(D^{o p p}\right)$. Les $F_{x}$-modules de Dieudonné $\left(V_{x}, \varphi_{x}\right)$ et réseaux $M_{x}$ vérifient les propriétés du lemme 9.3 de [L-R-S].

\subsection{Enoncé et preuve de l'unicité. -}

Proposition 5.2. - Tous les $\mathcal{D}$-faisceaux elliptiques spéciaux (de caractéristique o) sur $\overline{\kappa(o)}$ sont isogènes. L'algèbre $\operatorname{End}(V, \varphi, \lambda)$ est isomorphe à l'algèbre $\bar{D}$ déduite de $D$ en échangeant les invariants en o et $\infty$ (c'est-à-dire que $\bar{D}$ est non ramifiée en o, $\bar{D}_{\infty} \simeq D_{o}$ et $\bar{D}_{x} \simeq D_{x}$ pour tout $x \neq o, \infty)$.

Démonstration. - On montrera uniquement l'unicité à isogénie près (pas l'existence). Pour cela, on raisonne sur le $\varphi$-espace $(V, \varphi, \lambda)$ et sur la $\varphi$-paire $(\tilde{F}, \tilde{\Pi})$ associée $([\mathbf{L}-\mathbf{R}-\mathbf{S}]$ A.4).

Tout d'abord, $(V, \varphi, \lambda)$ est isotypique, i.e. isomorphe à $(W, \psi)^{n}$ pour un certain $\varphi$-espace irréductible $(W, \psi)$ et un certain entier positif $n$ (même raisonnement que [L-R-S] lemme 9.6). Localement en $x=\infty,\left(V_{\infty}, \varphi_{\infty}\right) \simeq\left(N_{d,-1}, \psi_{d,-1}\right)^{d}$ (loc. cit. lemme 9.8); en $x \neq o, \infty,\left(V_{x}, \varphi_{x}\right)$ est isotypique, de type $\left(N_{1,0}, \psi_{1,0}\right)$ (loc. cit. lemme B.6). Finalement, en $x=o,\left(V_{o}, \varphi_{o}\right) \simeq$ $\left(N_{d, 1}, \psi_{d, 1}\right)^{d}$, parce que les $\mathcal{D}$-faisceaux elliptiques considérés sont spéciaux.

Soit maintenant $\delta \in F$ vérifiant $o(\delta)=m, \infty(\delta)=-m$, et $\delta$ est une unité ailleurs. Un tel élément existe bien pour $m$ assez grand; on choisira de plus $m$ tel que $\tilde{\Pi}^{d m} \in \tilde{F}^{*}$. Montrons que $\tilde{\Pi}^{d m}$ et $\delta$ ont mêmes valuations en toutes les places de $\tilde{F}$.

Si $\tilde{o}$ est une place au-dessus de $o$, on a l'égalité $\operatorname{deg}(\tilde{o}) \tilde{o}(\tilde{\Pi}) /\left[\tilde{F}_{\tilde{o}}: F_{o}\right]=1 / d$ en vertu de L-R-S prop. B.4. Soit $\tilde{e}$ l'indice de ramification; l'égalité précédente se réécrit $\tilde{o}(\tilde{\Pi})=\tilde{e} / d$. Finalement $\tilde{o}\left(\tilde{\Pi}^{d m}\right)=m \tilde{e}$; c'est également $\tilde{o}(\delta)=\tilde{e} o(\delta)$. En la place $\infty$, on a de manière similaire $\operatorname{deg}(\tilde{\infty}) \tilde{\infty}(\tilde{\Pi}) /[\tilde{F}: F]=-1 / d$ (pour $\tilde{\infty}$ l'unique place de $\tilde{F}$ qui divise $\infty$, cf loc. cit. prop 9.9 (ii) $)$; on en conclut $\tilde{\infty}\left(\tilde{\Pi}^{d m}\right)=\tilde{\infty}(\delta)$ comme avant. Pour finir, $\tilde{\Pi}^{d m}$ et $\delta$ sont des unités en toutes les autres places qui ne divisent pas $\infty$ et $o$ (loc. cit. prop. B.4). 
Ainsi $\tilde{\Pi}^{d m} / \delta$ appartient à $\mathbb{F}_{q}^{*}$ et est une racine de l'unité; il existe donc $N$ et $N^{\prime}$ tels que $\tilde{\Pi}^{N}=\delta^{N^{\prime}}$. Or $\tilde{F}=F\left[\tilde{\Pi}^{N}\right]$ puisque $\tilde{\Pi}^{N} \in \tilde{F}^{*}$; finalement $\tilde{F}=F$ et la $\varphi$-paire $(\tilde{F}, \tilde{\Pi})=(F, \tilde{\Pi})$ est bien déterminée (à isomorphisme près) par les valuations de $\tilde{\Pi}: o(\tilde{\Pi})=1 / d, \infty(\tilde{\Pi})=-1 / d$ et $x(\tilde{\Pi})=0$ si $x \neq o, \infty$. Par le théorème de classification([L-R-S $]$ A.6), le $\varphi$-espace $(V, \varphi)$ est également déterminé à isomorphisme près.

Remarquons que $(V, \varphi)=(W, \psi)^{d}(i . e . n=d)$. En effet, $d(\tilde{\Pi})$ est le ppcm des dénominateurs de $\operatorname{deg}(\tilde{o}) \tilde{o}(\tilde{\Pi})$ et $\operatorname{deg}(\tilde{\infty}) \tilde{\infty}(\tilde{\Pi})$, en l'occurrence $d$. Or dans l'isomorphisme $\left(W_{\infty}, \psi_{\infty}\right) \simeq$ $\left(N_{d,-1}, \psi_{d,-1}\right)^{s}$, on a $d s=d(\tilde{\Pi})$, d'où $s=1$; puisque $\left(V_{\infty}, \varphi_{\infty}\right) \simeq\left(N_{d,-1}, \psi_{d,-1}\right)^{d}$, il faut $n=d$.

Il en résulte que l'algèbre d'endomorphismes End $(V, \varphi)$ est isomorphe à $M_{d}(H)$, où $H$ désigne l'algèbre centrale simple de dimension $d^{2}$ sur $F$ qui se ramifie exactement en $o$ et $\infty$ et dont les invariants sont les suivants : $\operatorname{inv}_{o}(H)=-1 / d$ et $\operatorname{inv}_{\infty}(H)=1 / d$. En effet, on connaît la nature locale de $(W, \psi)$; d'où l'assertion, sachant que End $\left(N_{d, r}, \psi_{d, r}\right)$ est une algèbre à division centrale sur $F_{x}$ d'invariant $-r / d$.

Finalement, se donner une action de $D$ revient à plonger $D^{o p p}$ dans $M_{d}(H)$. Soit $\bar{D}$ l'algèbre définie dans l'énoncé du théorème. Place par place, on vérifie que $D^{o p p} \otimes \bar{D}$ est isomorphe à $M_{d}(H)$. Cela prouve à la fois l'existence d'un plongement $D^{o p p} \hookrightarrow M_{d}(H)$ et le fait que l'algèbre End $(V, \varphi, \lambda)$ (laquelle s'identifie au commutant de $D^{o p p}$ dans $M_{d}(H)$ ) est isomorphe à $\bar{D}$.

L'unicité à isogénie des $\mathcal{D}$-faisceaux elliptiques spéciaux signifie que tous les plongements $D^{o p p} \hookrightarrow M_{d}(H)$ sont conjugués, ce qui résulte du théorème de Skolem-Noether.

\section{Deuxième PARTIE II \\ UNIFORMISATION « ̀̀ LA ČEREDNIK - DRINFELD »}

\section{Le problème de modules des $\mathcal{D}$-faisceaux elliptiques}

6.1. L'espace de modules $\mathcal{E} \ell_{X, \mathcal{D}, I}$ : rappels. - Fixons un sous-schéma fini non vide $I$ de $X-\{\infty\}$. Pour $z: S \rightarrow X^{\prime}$ un schéma au-dessus de $X^{\prime}=X-\{\infty\}-I-\mathcal{R}$, notons $\mathcal{E} l_{X, \mathcal{D}, I}(S)$ l'ensemble des classes d'isomorphisme de $\mathcal{D}$-faisceaux elliptiques sur $S$, de zéro $z$, munis d'une structure de niveau $I$.

Le premier résultat essentiel démontré dans $[\mathbf{L - R}-\mathbf{S}]$ est le :

Théorème 6.1. - Le foncteur $\mathcal{E} \ell_{X, \mathcal{D}, I}$ est représentable par un schéma $\mathcal{E} \ell_{X, \mathcal{D}, I}$ quasi-projectif sur $X^{\prime}$ :

$$
\mathcal{E} \ell_{X, \mathcal{D}, I} \stackrel{\text { zéro }}{\longrightarrow} X-\{\infty\}-I-\mathcal{R} .
$$

Ce schéma est lisse et purement de dimension relative $(d-1)$ au-dessus de $X^{\prime}$. Lorsque $D$ est une algèbre à division, c'est un $X^{\prime}$-schéma projectif.

Donnons un aperçu de la preuve : tout d'abord, à l'aide d'un résultat de semi-stabilité des fibrés $\mathcal{E}_{i}$, on montre l'existence d'un $\mathbb{F}_{q^{\text {-schéma }} \text { Vect }}{ }_{X, \mathcal{D}, I}$ classifiant les suites

$$
\stackrel{j}{\longrightarrow} \mathcal{E}_{-1} \stackrel{j}{\longrightarrow} \mathcal{E}_{0} \stackrel{j}{\longrightarrow} \mathcal{E}_{1} \stackrel{j}{\longrightarrow} \ldots
$$


de $\mathcal{D} \otimes \mathcal{O}_{S}$-modules localement libres de rang un, munis d'une structure $\mathcal{D}_{I} \otimes \mathcal{O}_{S} \simeq \mathcal{E}_{I}$ de niveau $I$, et vérifiant les conditions $(i)$, (ii) et $(i v)$ de la définition d'un $\mathcal{D}$-faisceau elliptique. Par ailleurs, il existe un $\mathbb{F}_{q^{-} \text {-schéma Heckex,D,I }}$ classifiant les diagrammes

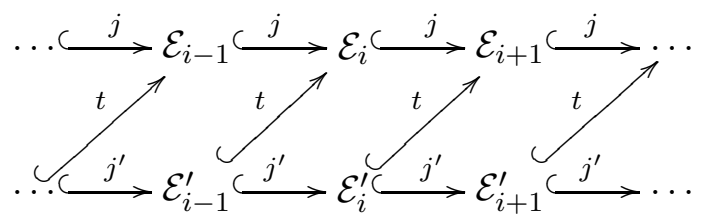

dont les deux rangs sont des suites de $\operatorname{Vect}_{X, \mathcal{D}, I}^{\bullet}$ et tels que les morphismes $t$ soient des injections $\mathcal{O}_{X \times S^{-}}$linéaires (compatibles à l'action de $\mathcal{D}$ et aux structures de niveau) qui vérifient la condition (iii) de la définition d'un $\mathcal{D}$-faisceau elliptique.

Les auteurs de [L-R-S] montrent que le schéma Hecke L,D,I $_{\text {L }}$ est lisse et quasi-projectif sur $\mathbb{F}_{q}$, et que le morphisme

$$
\operatorname{Hecke}_{X, \mathcal{D}, I} \stackrel{\left(z, r_{1}\right)}{\longrightarrow}(X-\{\infty\}-I-\mathcal{R}) \times \operatorname{Vect}_{X, \mathcal{D}, I}^{\bullet},
$$

donné par le morphisme zéro et par le premier rang, est lisse de dimension relative $d-1$. On conclut alors à la représentatibilité de $\mathcal{E} \ell_{X, \mathcal{D}, I}$ par un schéma quasi-projectif lisse de dimension relative $d-1$ en remarquant que c'est le produit fibré :

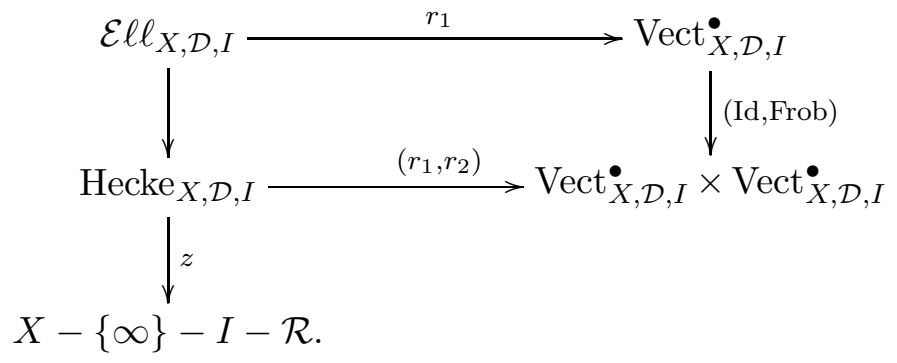

La dernière assertion du théorème se démontre en vérifiant le critère valuatif de propreté, lequel résulte du lemme suivant (« potentiellement bonne réduction ») :

Lemme 6.2. - Supposons que $D$ soit une algèbre à division. Soit $\mathcal{O} \supseteq \mathbb{F}_{q}$ un anneau de valuation discrète de corps des fractions noté $K$, et soit $\mathcal{E}=\left(\mathcal{E}_{i}, j, t\right)$ un point de $\mathcal{E} \ell_{X, \mathcal{D}, I}(K)$. Alors il existe une extension finie $K^{\prime}$ de $K$ et un point $\tilde{\mathcal{E}}=\left(\tilde{\mathcal{E}}_{i}, \tilde{j}, \tilde{t}\right)$ de $\mathcal{E} \ell_{X, \mathcal{D}, I}$ à valeurs dans la clôture intégrale $\mathcal{O}^{\prime}$ de $\mathcal{O}$ dans $K^{\prime}$, telle que l'image de $\tilde{\mathcal{E}}$ dans $\mathcal{E} l l_{X, \mathcal{D}, I}\left(K^{\prime}\right)$ coïncide avec celle de $\mathcal{E}$.

Pour démontrer ce lemme, Laumon, Rapoport et Stuhler utilisent un théorème de réduction semi-stable ([Dr7 $\S 3)$ développé par Drinfeld dans un travail sur la compactification des espaces de modules de Shtukas.

On a donc :

Proposition 6.3. - Supposons que D soit une algèbre à division. Alors le morphisme (3) est propre.

6.2. Prolongement de $\mathcal{E} \ell_{X, \mathcal{D}, I}$ au-dessus de $o$. - Rappelons que $o$ désigne une place de $F$ telle que $D_{o}$ est un corps gauche d'invariant $1 / d$ sur $F_{o}$ et $I$ un sous-schéma fermé fini non vide de $X-\{\infty\}$ ne contenant pas o. Afin d'alléger les notations, on notera toujours $\mathcal{E} \ell_{X, \mathcal{D}, I}(S)$ l'ensemble des classes d'isomorphisme de $\mathcal{D}$-faisceaux elliptiques spéciaux définis sur un schéma $S$ et munis d'une structure de niveau $I$. 
Théorème 6.4. - Le foncteur $\mathcal{E} \ell_{X, D}, I$ est représentable par un schéma projectif sur $(X-\{\infty\}-I-\mathcal{R}) \cup\{o\}$ qui prolonge le schéma $\mathcal{E} \ell_{X, \mathcal{D}, I}$.

Démonstration. - Examinant les divers arguments avancés dans la preuve du théorème 6.1 dont on a rappelé les étapes, on constate que l'hypothèse sur le zéro $z$ (qui évite le lieu de ramification $\mathcal{R}$ de l'algèbre $D$ ) intervient uniquement au niveau du schéma Hecke $X, \mathcal{D}, I$ au-dessus de $(X-\{\infty\}-I-\mathcal{R}) \times \operatorname{Vect}_{X, \mathcal{D}, I}^{\bullet}$; rappelons la situation : un objet de $X^{\prime} \times \operatorname{Vect}_{X, \mathcal{D}, I}^{\bullet}(S)$ correspond à un morphisme $z: S \rightarrow X^{\prime}$ et à une suite $\ldots \hookrightarrow \mathcal{E}_{i} \stackrel{j}{\hookrightarrow} \mathcal{E}_{i+1} \hookrightarrow \ldots$ de $\mathcal{D} \otimes \mathcal{O}_{S^{-}}$ modules localement libres de rang un munis d'une structure de niveau $I$. Compléter cette donnée par une seconde rangée $\left(\mathcal{E}_{i}^{\prime}\right)$ avec des morphismes $t_{i}: \mathcal{E}_{i}^{\prime} \rightarrow \mathcal{E}_{i}$ satisfaisant toutes les conditions requises est équivalent à se donner un sous $\mathcal{O}_{X \times S}$-module $\mathcal{E}^{\prime} \subset \mathcal{E}_{0}$ localement libre de rang $d^{2}$, laissé stable par $\mathcal{D}$, et tel que $\mathcal{E}_{0} / \mathcal{E}^{\prime}$ soit localement libre de rang $d$ sur $\mathcal{O}_{S}$ et de support le graphe $\Gamma_{z}$ de $z$ (parce que $z(S) \not \supset \infty$, la donnée des morphismes $t_{i}$ est équivalente à la donnée de $\left.t_{0}\right)$.

On raisonne alors comme Lafforgue pour les Chtoucas de Drinfeld (cf. [L] I §2 lemme 8) : considérons le foncteur qui à $S^{\prime} \rightarrow S$ associe l'ensemble des suites exactes

$$
0 \rightarrow \mathcal{E}^{\prime} \rightarrow \mathcal{E}_{0} \otimes \mathcal{O}_{S} \mathcal{O}_{S^{\prime}} \rightarrow \mathcal{Q} \rightarrow 0
$$

où $\mathcal{E}^{\prime}$ est localement libre de rang $d^{2}$ comme $\mathcal{O}_{X \times S^{\prime}}$-module, et où $\mathcal{Q}$ est supporté par le graphe du composé $z^{\prime}: S^{\prime} \rightarrow S \rightarrow X$ et est localement libre de rang $d$ sur $\mathcal{O}_{S^{\prime}}$. En posant $\mathcal{Q}^{\prime}=\left(\operatorname{Id}, z^{\prime}\right)^{*} \mathcal{Q}$, cela revient à considérer l'ensemble des $\mathcal{O}_{S^{\prime}}$-modules quotients $\mathcal{Q}^{\prime} \operatorname{de}\left(\mathrm{Id}, z^{\prime}\right)^{*} \mathcal{E}_{0} \otimes_{\mathcal{O}_{S}} \mathcal{O}_{S^{\prime}}$ qui sont localement libres de rang $d$. Ce foncteur est donc représentable par un morphisme $S_{1} \rightarrow S$ qui est grassmannien, donc projectif. Notons $\mathcal{E}_{1}^{\prime}$ et $\mathcal{Q}_{1}$ les faisceaux canoniques sur $X \times S_{1}$. Il s'agit alors de regarder, pour tout morphisme de schéma $S^{\prime} \rightarrow S_{1}$, quand est-ce que l'action de $\mathcal{D} \otimes \mathcal{O}_{S^{\prime}}$ se prolonge à $\mathcal{E}_{1}^{\prime} \otimes_{\mathcal{O}_{S_{1}}} \mathcal{O}_{S^{\prime}}$ et à $\mathcal{Q}_{1} \otimes_{\mathcal{O}_{S_{1}}} \mathcal{O}_{S^{\prime}}$. Cela revient à demander que soit annulé l'homomorphisme $\mathcal{E}_{1}^{\prime} \otimes_{\mathcal{O}_{X}} \mathcal{D} \rightarrow \mathcal{Q}_{1}$ par le changement de base $S^{\prime} \rightarrow S_{1}$. Cette condition est représentable par une immersion fermée $S_{2} \hookrightarrow S_{1}$ : cela résulte du lemme suivant.

Lemme 6.5 (cf. [L I $\$ 2$ lemme 3). - Soient $Z \rightarrow Y$ un morphisme projectif de schémas, $\mathcal{F}, \mathcal{G}$ deux faisceaux cohérents sur $Z$ tels que $\mathcal{G}$ soit plat sur $\mathcal{O}_{Y}$ et $\alpha: \mathcal{F} \rightarrow \mathcal{G}$ un homomorphisme global.

Alors il existe une immersion fermée $Y_{0} \hookrightarrow Y$ telle que, pour tout morphisme de schémas $Y^{\prime} \rightarrow Y$, l'homomorphisme $\alpha$ soit annulé par le changement de base $Y^{\prime} \rightarrow Y$ si et seulement si $Y^{\prime} \rightarrow Y$ se factorise à travers $Y_{0}$.

Cela montre que Hecke $_{X, \mathcal{D}, I} \rightarrow\left(X^{\prime} \cup\{o\}\right) \times$ Vect $_{X, \mathcal{D}, I}^{\bullet}$ est représentable et quasi-projectif. Remarquons que la restriction de ce morphisme au-dessus de l'ouvert $X^{\prime} \times \operatorname{Vect}_{X, \mathcal{D}, I}$ est lisse de dimension relative $d-1$ : c'est l'argument de [L-R-S] : parce que $X^{\prime} \cap \mathcal{R}=\emptyset$, alors $z^{*} \mathcal{D}$ est une algèbre d'Azumaya (c'est-à-dire isomorphe à $\mathrm{M}_{d}\left(\mathcal{O}_{S}\right)$ localement pour la topologie étale) lorsque $z(S) \subset X^{\prime}$. Or si $S^{\prime} \rightarrow S$ est un morphisme de schémas, se donner un $\mathcal{O}_{S^{\prime}}$-module quotient $\mathcal{Q}^{\prime}$ de $(\mathrm{Id}, z)^{*} \mathcal{E} \otimes \mathcal{O}_{S} \mathcal{O}_{S^{\prime}}$ sur $S^{\prime}$ qui soit localement libre de rang $d$ et compatible avec l'action à droite de $\mathrm{M}_{d}\left(\mathcal{O}_{S^{\prime}}\right)$ revient, par équivalence de Morita, à se donner un $\mathcal{O}_{S^{\prime}}$-module quotient d'un certain fibré de rang $d$ qui soit inversible. On voit donc que $S_{2}$ est l'espace projectif sur $S$ associé à ce fibré; il est lisse et de dimension relative $d-1$.

Mis à part le point précédent, les autres arguments de [L-R-S] s'appliquent à la lettre. Comme la condition spéciale est ouverte (et fermée : voir les remarques suivant l'énoncé de la condition spéciale), on en conclut la représentabilité du foncteur $\mathcal{E} \ell_{X, \mathcal{D}, I}$ par un schéma quasi-projectif $\operatorname{sur}(X-\{\infty\}-I-\mathcal{R}) \cup\{o\}$. La projectivité résulte alors du lemme 6.2, qui reste en fait valable 
dans ce nouveau contexte (c'est-à-dire pour $\mathcal{O}$ une $\mathcal{O}_{o}$-algèbre de valuation discrète ; avec les notations de ce lemme, $\tilde{\mathcal{E}}$ est bien spécial, en vertu de 3.2 remarque (b)).

Remarques. - 1. Si on oublie la condition "spéciale ", le foncteur obtenu est encore représentable et projectif sur $X^{\prime} \cup\{o\}$. Cependant, sans cette condition, il n'y a pas d'uniformisation rigide-analytique en la place o.

2. Le schéma $\mathcal{E} \ell_{X, \mathcal{D}, I}$ prolongé n'est plus lisse (sur $X^{\prime} \cup\{o\}$ ). Le lecteur notera que la théorie des déformations étudiée au paragraphe 4.1 est absolue et non relative au-dessus de $X$.

3. En vertu de la remarque 3.2 (b), la condition spéciale est nécessaire afin que le schéma de module obtenu soit plat au-dessus de $\operatorname{Spec} \mathcal{O}_{o}$. On peut montrer, par exemple en utilisant l'analogue du théorème de Čerednik que nous allons prouver, que tel est bien le cas.

6.3. Les opérateurs de Hecke. - Soit $\mathbb{A}$ désigne l'anneau des adèles de $F$ et $\mathcal{O}_{\mathbb{A}}=$ $\prod_{x \in|X|} \mathcal{O}_{x}$ son sous-anneau des entiers; nous notons $\mathbb{A}^{\infty}=\mathbb{A} / F_{\infty}, \mathcal{O}^{\infty}=\mathcal{O}_{\mathbb{A}} / \mathcal{O}_{\infty}$ et $\mathcal{D}^{\infty}=$ $\mathcal{D} \otimes_{\mathcal{O}_{X}} \mathcal{O}^{\infty}$. Considérons les schémas $\mathcal{E} \ell_{X, \mathcal{D}, I}$; pour $I^{\prime} \supset I$, la restriction de la structure de niveau définit une projection (finie étale au-dessus de $X-\{\infty\}-I^{\prime}$ ) de $\mathcal{E} \ell_{X, \mathcal{D}, I^{\prime}}$ sur $\mathcal{E} \ell_{X, \mathcal{D}, I}$. Nous désignons par $\mathcal{E} \ell \ell_{X, \mathcal{D}}^{\infty}$ le système projectif obtenu ( $I$ décrivant $\left.X \backslash\{\infty\}\right)$; une section de $\mathcal{E} \ell_{X, \mathcal{D}}^{\infty}$ au-dessus d'un schéma $S$ est équivalente à la donnée d'un $\mathcal{D}$-faisceau elliptique $\left(\mathcal{E}_{i}, j, t\right)$ sur $S$, muni d'un isomorphisme $\mathcal{D}$-linéaire $\iota^{\infty}: \mathcal{D}^{\infty} \otimes \mathcal{O}_{S} \stackrel{\sim}{\rightarrow} \mathcal{E}_{i} \otimes_{\mathcal{O}_{X \times S}}\left(\mathcal{O}^{\infty} \otimes \mathcal{O}_{S}\right)$ (compatible avec les structures de Frobenius données par Id $\otimes F r_{S}$ à gauche et $t_{i}$ à droite).

Désignant par $D^{*}$ le groupe multiplicatif de l'algèbre $D$, tel que $D^{*}(B)=\left(D \otimes_{F} B\right)^{*}$ pour toute $F$-algèbre $B$, le système projectif $\mathcal{E} \ell \ell_{X, \mathcal{D}}^{\infty}$ est muni d'une action à droite de $D^{*}\left(\mathbb{A}^{\infty}\right)$ (cf. [L-R-S] $\S 7)$. Tout d'abord, on définit l'action du semi-groupe $\Gamma=D^{*}\left(\mathbb{A}^{\infty}\right) \cap \mathcal{D}^{\infty}$ : un élément $g$ de $\Gamma$ induit un endomorphisme de $\mathcal{D}^{\infty}$ (par multiplication à gauche) et donc (via $\iota^{\infty}$ ) de $\mathcal{E}_{i} \otimes_{\mathcal{O}_{X \times S}}\left(\mathcal{O}^{\infty} \otimes \mathcal{O}_{S}\right)$. A ces données, on associe alors le $\mathcal{D}$-faisceau elliptique $\left(\mathcal{E}_{i}^{\prime}, j_{i}^{\prime}, t_{i}^{\prime}\right)$ qui s'insère dans le diagramme cartésien suivant :

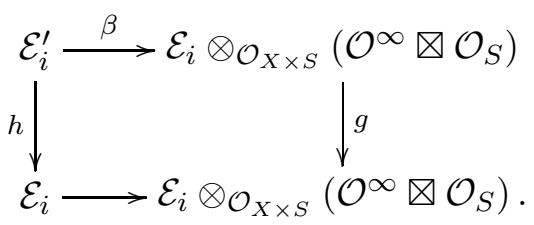

Il est muni de la structure de niveau $\iota^{\prime \infty}=(\beta \otimes 1)^{-1} \circ \iota^{\infty}$. D'autre part, $\left(F^{*} \cap \mathcal{O}_{\infty}^{*}\right) \backslash\left(\mathbb{A}^{\infty}\right)^{*}$, identifié au groupe de Picard Pic $\operatorname{Pi}_{X}^{\infty}$ des faisceaux inversibles $\mathcal{L}$ sur $X$ (tels que $\mathcal{L}_{\infty}=\mathcal{O}_{\infty}$ ) munis d'une structure de niveau $\iota_{\mathcal{L}}^{\infty}: \mathcal{O}^{\infty} \stackrel{\sim}{\rightarrow} \mathcal{L} \otimes_{\mathcal{O}_{X}} \mathcal{O}^{\infty}$, opère sur $\mathcal{E} \ell \ell_{X, \mathcal{D}}$ par tensorisation à gauche. Finalement, on vérifie que les actions de $\Gamma$ et $\left(\mathbb{A}^{\infty}\right)^{*}$ ainsi définies coïncident sur leur intersection $\left(\mathbb{A}^{\infty}\right)^{*} \cap \mathcal{O}^{\infty}$, d'où une action du groupe $D^{*}\left(\mathbb{A}^{\infty}\right)$ qu'ils engendrent.

Pour $I \subset X \backslash\{\infty\}$ un sous-schéma fermé fini non vide et $\mathfrak{I} \subset \mathcal{O}_{X}$ le faisceau d'idéaux correspondant, on pose $K_{I}^{\infty}=\operatorname{Ker}\left[\left(\mathcal{D}^{\infty}\right)^{*} \rightarrow\left(\mathfrak{I}^{\infty} \mathcal{D}^{\infty} \backslash \mathcal{D}^{\infty}\right)^{*}\right]$; c'est un sous-groupe ouvert compact de $D^{*}\left(\mathbb{A}^{\infty}\right)$. Considérons les schémas $\mathcal{E} \ell \ell_{I, F_{o}}:=\mathcal{E} \ell \ell_{X, \mathcal{D}, I} \times_{X} \operatorname{Spec} F_{o}$ ainsi que leur limite projective $\mathcal{E} \ell \ell_{F_{o}}$ lorsque $I$ varie. On a $\mathcal{E} \ell \ell_{I, F_{o}}=\mathcal{E} \ell \ell_{F_{o}} / K_{I}^{\infty}$. L'action de $g^{\infty} \in D^{*}\left(\mathbb{A}^{\infty}\right)$ dont il est question plus haut peut également se décrire en terme de correspondances de Hecke : la double classe de 
$g^{\infty}$ modulo $K_{I}^{\infty}$ induit la correspondance géométrique de $\mathcal{E} \ell \ell_{I, F_{o}}$ sur $F_{o}$ :

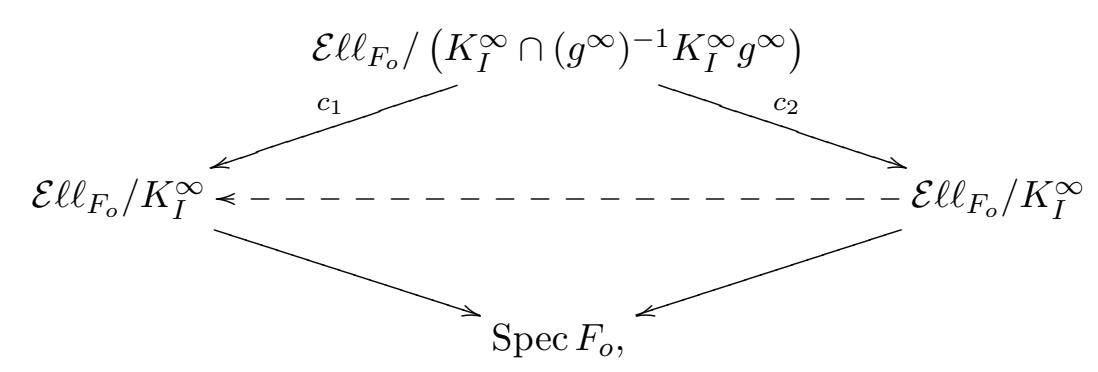

avec $c_{1}$ le morphisme qui provient de l'inclusion $K_{I}^{\infty} \cap\left(g^{\infty}\right)^{-1} K_{I}^{\infty} g^{\infty} \subset K_{I}^{\infty}$, tandis que $c_{2}$ provient de l'action de $\left(g^{\infty}\right)^{-1}$ sur $\mathcal{E} \ell \ell_{F_{o}}$ et de l'injection $u \mapsto g^{\infty} u\left(g^{\infty}\right)^{-1}$ de $K_{I}^{\infty} \cap\left(g^{\infty}\right)^{-1} K_{I}^{\infty} g^{\infty}$ dans $K_{I}^{\infty}$. Lorsque la composante $g_{o}^{\infty}$ est triviale, cette correspondance s'étend en une correspondance de $\mathcal{E} \ell \ell_{X, \mathcal{D}, I} \times_{X} \operatorname{Spec} \mathcal{O}_{o} \operatorname{sur} \operatorname{Spec} \mathcal{O}_{o}$, avec des morphismes $c_{1}$ et $c_{2}$ étales finis.

\section{Le Théorème de Drinfeld}

Dans toute cette partie, $K$ désigne un corps local non archimédien, $\mathcal{O}$ l'anneau des entiers de $K$ et $\pi$ une uniformisante de $\mathcal{O}$. Soient $\kappa=\mathcal{O} / \pi \mathcal{O}$ le corps résiduel, $p$ la caractéristique de $\kappa$ et $q=p^{r}$ son ordre. On note $C$ le complété d'une clôture algébrique de $K$ et || la norme sur $C$ normalisée par $|\pi|=q^{-1}$; la valuation (normalisée) est donnée par $v(x)=\log _{q}|x|$. On choisit également une clôture algébrique $\bar{\kappa}$ de $\kappa$ et note $\mathcal{O}^{n r}$ l'extension non ramifiée maximale de $\mathcal{O}$, de corps résiduel $\bar{\kappa}$.

7.1. Le schéma formel $\widehat{\Omega}^{d}$. — Pour tout entier $d \geq 1$, Drinfeld a construit dans [Dr1] une variété rigide-analytique $\Omega^{d}$ sur $K$ dont l'ensemble des points à valeur dans une extension finie $L \subset C$ de $K$ est

$$
\mathbb{P}^{d-1}(L)-\bigcup_{H / K} H(L)
$$

(où $H$ parcourt l'ensemble des hyperplans rationnels sur $K$ de $\mathbb{P}^{d-1}$ ). C'est un analogue nonarchimédien des espaces hermitiens symétriques, pour le cas du groupe linéaire $\mathrm{GL}_{d}$ (pour $d=2$, on retrouve l'analogue du demi-plan de Poincaré).

Le $\mathcal{O}$-schéma formel $\widehat{\Omega}^{d}$ (construit par Deligne) est un modèle formel de $\Omega^{d}$ : sa fibre générique au sens de Raynaud ([Ra2 $)$ ) est $\Omega^{d}$. Il est muni d'une action de $\mathrm{PGL}_{d}(K)$ (qui est visible au moins ensemblistement). Le lecteur trouvera dans [Bo-Ca ou $\mathbf{G e}$ plusieurs descriptions fonctorielles de $\widehat{\Omega}^{d}$. Voir également [Bo-Ca ou [De-Hu] $\S 3$ pour une description de la structure rigide-analytique sur $\Omega^{d}$.

7.2. Enoncé du théorème. - A partir de maintenant, fixons un entier $d \geq 2$, donnons-nous un corps gauche $D \subset C$ d'invariant $1 / d$ sur $K$ et notons $\mathcal{O}_{D}$ son anneau des entiers. D'après la proposition 3.4, tous les $\mathcal{O}_{D}$-modules formels spéciaux de hauteur $d^{2}$ sur $\bar{\kappa}$ sont isogènes; soit $\Phi$ l'un d'entre eux, que l'on fixe, ainsi qu'un isomorphisme

$$
\mathrm{GL}_{d}(K)=\operatorname{Aut}_{D}^{0}(\Phi) .
$$

Définition 7.1. - Soit $\overline{\mathcal{N} i l p}$ la catégorie des $\mathcal{O}^{\text {nr }}$-algèbres où l'image de $\pi$ est nilpotente. On définit un foncteur $\bar{G}$ sur $\overline{\mathcal{N} i l p}$ en associant à $B \in \mathrm{Ob} \overline{\mathcal{N} i l p}$ l'ensemble $\bar{G}(B)$ des classes d'isomorphie de couples $(H, \rho)$ consistant en :

1. un $\mathcal{O}_{D}$-module formel spécial $H$ de hauteur $d^{2}$ sur $B$; 
2. une quasi-isogénie de hauteur zéro $\rho: \Phi_{\bar{B}} \rightarrow H_{\bar{B}}$, où $\bar{B}=B / \pi B$ et $\Phi_{\bar{B}}$ est déduit de $\Phi$ par changement de base (on rigidifie la fibre spéciale uniquement).

Rappel : une quasi-isogénie $\rho: \Phi_{B / \pi B} \rightarrow H_{B / \pi B}$ (de hauteur zéro) est un élément de $\operatorname{Hom}_{\mathcal{O}_{D}}\left(\Phi_{\bar{B}}, H_{\bar{B}}\right) \otimes_{\mathcal{O}} K$ tel que $\pi^{n} \rho$ soit, pour $n$ entier suffisamment grand, une isogénie (de hauteur $\left.d^{2} n\right) ; \rho$ est alors inversible dans $\operatorname{Hom}_{\mathcal{O}_{D}}\left(\Phi_{\bar{B}}, H_{\bar{B}}\right) \otimes_{\mathcal{O}} K$ (voir [Zi2] Kapitel V). Le résultat fondamental de Drinfeld est le suivant :

Théorème 7.2. - Le foncteur $\bar{G}$ est représentable par le $\widehat{\mathcal{O}}^{n r}$-schéma formel $\widehat{\Omega}^{d} \hat{\otimes}_{\mathcal{O}} \widehat{\mathcal{O}}^{n r}$

Définition 7.3. - Soit $\mathcal{N} i l p$ la catégorie des $\mathcal{O}$-algèbres où l'image de $\pi$ est nilpotente. On définit un foncteur $G$ sur $\mathcal{N} i l p$ en associant à $B \in$ Ob $\mathcal{N} i l p$ l'ensemble $G(B)$ des couples formés :

1. d'un $\kappa$-homomorphisme $\psi: \bar{\kappa} \rightarrow B / \pi B$.

2. d'une classe d'isomorphie de couples $(H, \rho)$ consistant en :

- un $\mathcal{O}_{D}$-module formel spécial $H$ de hauteur $d^{2}$ sur $B$;

- une quasi-isogénie de hauteur zéro $\rho: \psi_{*} \Phi \rightarrow H_{B / \pi B}$.

Le foncteur $G$ n'est autre que le foncteur déduit de $\bar{G}$ par restriction des scalaires de $\mathcal{O}^{n r}$ à $\mathcal{O}$. Ainsi :

Théorème 7.4. - (cf. Dr4 §2) Le foncteur $G$ est représentable par le $\mathcal{O}$-schéma formel $\widehat{\Omega}^{d} \hat{\otimes}_{\mathcal{O}} \widehat{\mathcal{O}}^{n r}$.

7.3. Action des groupes $\mathrm{GL}_{d}(K)$ et $D^{*}$. - On rappelle que $F r: \bar{\kappa} \rightarrow \bar{\kappa}$ désigne l'homomorphisme de Frobenius $\operatorname{Fr}(x)=x^{q}$ et Frob $: F r_{*}^{-1} \Phi \rightarrow \Phi$ l'isogénie de Frobenius (de hauteur égale à la dimension $d$ de $\Phi)$. Via l'identification $\mathrm{GL}_{d}(K)=\operatorname{Aut}_{D}^{0}(\Phi)$, un élément $g$ de $\mathrm{GL}_{d}(K)$ définit une quasi-isogénie de $\Phi$ de hauteur $d n$ si $v(\operatorname{det} g)=n$. Ainsi $g^{-1} \circ$ Frob $^{n}: F r_{*}^{-n} \Phi \rightarrow \Phi$ est une quasi-isogénie de hauteur nulle. On définit une action de $\mathrm{GL}_{d}(K)$ sur le foncteur $G$ en posant, pour $B \in \mathrm{Ob} \mathcal{N} i l p$ et $(\psi, H, \rho)$ représentant un élément de $G(B)$ :

$$
g \cdot(\psi, H, \rho)=\left(\psi \circ F r^{-n}, H, \rho \circ \psi_{*}\left(g^{-1} \circ \operatorname{Frob}^{n}\right)\right) .
$$

Notons $\widetilde{F r}: \mathcal{O}^{n r} \rightarrow \mathcal{O}^{n r}$ le relèvement du $\kappa$-homomorphisme $\mathrm{Fr}: \bar{\kappa} \rightarrow \bar{\kappa}$ en un $\mathcal{O}_{-}$ homomorphisme.

Proposition 7.5. - (cf. [Dr4] $\S 2$ ou [Bo-Ca], théorème 9.3) L'action de $\mathrm{GL}_{d}(K)$ sur le foncteur $G$ correspond à l'action sur le schéma formel $\widehat{\Omega}^{d} \hat{\otimes}_{\mathcal{O}} \widehat{\mathcal{O}}^{n r}$ définie par l'action naturelle de $\mathrm{PGL}_{d}(K)$ sur $\widehat{\Omega}^{d}$ et l'action $g \mapsto \widetilde{F r}^{-v(\operatorname{det} g)} \operatorname{sur} \mathcal{O}^{n r}$.

Soit $N_{D / K}: D^{*} \rightarrow K^{*}$ la norme réduite : tout élément de $D^{*}$ s'écrit $g=\Pi^{n} \cdot g_{0}$ avec $g_{0} \in \mathcal{O}_{D}^{*}$ et $n=v\left(N_{D / K} g\right)$. Pour tout $g \in D^{*}$, notons ${ }^{g} H$ le $\mathcal{O}_{D}$-module formel qui coïncide avec $H$ en tant que $\mathcal{O}$-module, mais où l'action de $a \in \mathcal{O}_{D}$ sur ${ }^{g} H$ est identique à l'action de $g^{-1} a g$ sur $H$. L'action de $\mathcal{O}_{D}$ sur $\Phi$ associe à $g^{-1}$ une quasi-isogénie $\mathcal{O}_{D}$-équivariante de hauteur $-d n$ si $v\left(N_{D / K} g\right)=n$. Ainsi $g^{-1} \circ \operatorname{Frob}^{n}: F r_{*}^{-n} \Phi \rightarrow{ }^{g} \Phi$ est une quasi-isogénie $\mathcal{O}_{D}$-équivariante de hauteur nulle. On définit une action de $D^{*}$ sur le foncteur $G$ en posant, pour $B \in$ Ob $\mathcal{N}$ ilp et $(\psi, H, \rho)$ représentant un élément de $G(B)$ :

$$
g \cdot(\psi, H, \rho)=\left(\psi \circ \mathrm{Fr}^{-n},{ }^{g} H, \rho \circ \psi_{*}\left(g^{-1} \circ \mathrm{Frob}^{n}\right)\right) .
$$

Proposition 7.6. - (cf. [Dr4] §2 ou [Bo-Ca], théorème 9.5) L'action de $D^{*}$ sur le foncteur $G$ correspond à l'action sur le schéma formel $\widehat{\Omega}^{d} \hat{\otimes}_{\mathcal{O}} \widehat{\mathcal{O}}^{n r}$ définie par l'action $g \mapsto \widetilde{F r}^{-v\left(N_{D / K} g\right)}$ $\operatorname{sur} \mathcal{O}^{n r}$. 
7.4. Construction d'un système de revêtements $\Sigma_{n}^{d}$ de $\Omega^{d} \hat{\otimes}_{K} \widehat{K}^{n r}$. - Soit $Y$ le $\mathcal{O}_{D^{-}}$ module formel spécial universel sur $\widehat{\Omega}^{d} \hat{\otimes}_{\mathcal{O}} \widehat{\mathcal{O}}^{n r}$. Pour tout entier $n$, le noyau $Y_{n}^{d}$ de l'isogénie $Y\left(\pi^{n}\right)$ est un schéma formel en groupes fini et plat d'ordre $q^{n d^{2}}$, muni d'une action de $\mathcal{O}_{D} / \pi^{n} \mathcal{O}_{D}$. Soit $\mathcal{Y}_{n}^{d}$ la fibre générique au sens de Raynaud ([Ra2 $]$ ) de $Y_{n}^{d}$. Les espaces rigides

$$
\Sigma_{n}^{d}=\operatorname{Isom}_{\mathcal{O}_{D}}\left(\mathcal{O}_{D} / \pi^{n} \mathcal{O}_{D}, \mathcal{Y}_{n}^{d}\right)
$$

constituent un système projectif (via $\pi: \mathcal{Y}_{n+1}^{d} \rightarrow \mathcal{Y}_{n}^{d}$ ) de revêtements $\Sigma_{n}^{d} \rightarrow \Omega^{d} \otimes_{K} \widehat{K}^{n r}$ étales galoisiens de groupe de Galois $\left(\mathcal{O}_{D} / \pi^{n} \mathcal{O}_{D}\right)^{*}($ cf. [Dr1] $)$.

Enfin, les actions de $\mathrm{GL}_{d}(K)$ et $D^{*} \operatorname{sur} \Omega^{d} \otimes_{K} K_{d}$ se relèvent en une action sur les revêtements $\Sigma_{n}^{d}$

\section{Analogue du théorème de Čerednik-Drinfeld}

8.1. Notations. - Considérons l'algèbre à division $\bar{D}$ définie dans la proposition 5.2 (déduite de $D$ en échangeant les invariants en les places $o$ et $\infty)$. Nous notons $\bar{D}^{*}$ le groupe multiplicatif de l'algèbre $\bar{D}$; c'est un groupe réductif sur $F$ tel que $\bar{D}^{*}(R)=(\bar{D} \otimes R)^{*}$ pour toute $F$-algèbre $R$. En particulier, $\bar{D}^{*}\left(F_{o}\right) \simeq \mathrm{GL}_{d}\left(F_{o}\right)$ car $\bar{D}$ est déployé en la place $o$.

Pour $\mathcal{E}$ un $\mathcal{D}$-faisceau elliptique de caractéristique $o$ (défini sur $S=\operatorname{Spec} \overline{\kappa(o)}$ ), on note toujours $(V(\mathcal{E}), \varphi, \lambda)$ sa fibre générique; c'est un $\varphi$-espace muni via $\lambda$ d'une action de $D$ à droite (cf. [5.1). Localement en chaque place $x$ de $F$, on a les $F_{x}$-modules de Dieudonné sur $\overline{\kappa(o)}$ correspondant, avec action de $D_{x}$, notés $\left(V_{x}(\mathcal{E}), \varphi_{x}, \lambda_{x}\right)=(V(\mathcal{E}), \varphi, \lambda) \hat{\otimes}_{F} F_{x}$. On dispose également des $\mathcal{D}_{x}$-réseaux $M_{x}=H^{0}\left(\operatorname{Spec}\left(\mathcal{O}_{x} \hat{\otimes} \overline{\kappa(o)}\right), \mathcal{E}_{0}\right)$ stables sous $D_{x}$. Ces derniers vérifient les propriétés du lemme 9.3 de [L-R-S] ; en vertu de loc. cit. lemme B.6, l'application canonique $V_{x}^{\varphi_{x}}(\mathcal{E}) \hat{\otimes} \bar{\kappa}(o) \rightarrow V_{x}(\mathcal{E})$ est bijective pour tout $x \neq \infty, o$ (notant $V_{x}^{\varphi_{x}}(\mathcal{E})$ l'espace des invariants sous $\left.\varphi_{x}\right)$.

Considérons le produit restreint $\left(V^{\infty, o}(\mathcal{E}), \varphi^{\infty, o}\right)$ des $\left(V_{x}(\mathcal{E}), \varphi_{x}\right)$ relativement aux $M_{x}$ et en dehors des places $\infty, o$; il est muni d'une action à droite de $D\left(\mathbb{A}^{\infty, o}\right)$ donnée par le produit restreint $\lambda^{\infty, o}$ des $\lambda_{x}$. Il résulte des rappels précédents que l'application canonique

$$
\left(\left(V^{\infty, o}(\mathcal{E})\right)^{\varphi^{\infty, o}} \hat{\otimes} \overline{\kappa(o)}, \operatorname{Id}_{V^{\infty, o}} \hat{\otimes} F r\right) \longrightarrow\left(V^{\infty, o}(\mathcal{E}), \varphi^{\infty, o}\right)
$$

est un isomorphisme, où $F r$ désigne le Frobenius $\operatorname{Fr}(x)=x^{q}$ de $\overline{\kappa(o)}$. Soit d'autre part $V_{x}=D_{x}$ vu comme $D_{x}$-module à droite et $V^{\infty, o}$ le produit restreint des $V_{x}$ relativement aux ordres $\mathcal{D}_{x} \subset D_{x}$ pour $x \neq \infty, o$ (c'est également $D\left(\mathbb{A}^{\infty, o}\right)$ vu comme $D\left(\mathbb{A}^{\infty, o}\right)$-module à droite). Clairement, $\left(V^{\infty, o}(\mathcal{E})\right)^{\varphi^{\infty, o}}$ est un $D\left(\mathbb{A}^{\infty, o}\right)$-module libre de rang un, donc isomorphe à $V^{\infty, o}$. Fixons un tel isomorphisme; il en résulte un isomorphisme

$$
\text { End }\left(V^{\infty, o}(\mathcal{E}), \varphi^{\infty, o}, \lambda^{\infty, o}\right) \simeq \operatorname{End}_{D\left(\mathbb{A}^{\infty, o}\right)}\left(V^{\infty, o}\right)=D\left(\mathbb{A}^{\infty, o}\right) .
$$

En vertu de la proposition 5.2 , les endomorphismes de la fibre générique $(V(\mathcal{E}), \varphi, \lambda)$ s'identifient à $\bar{D}$; le choix d'un tel isomorphisme détermine également un isomorphisme $\bar{D}\left(\mathbb{A}^{\infty, o}\right) \simeq$ End $\left(V^{\infty, o}(\mathcal{E}), \varphi^{\infty, o}, \lambda^{\infty, o}\right)$. Finalement, ces divers choix conduisent à un isomorphisme $\bar{D}\left(\mathbb{A}^{\infty, o}\right) \simeq$ $D\left(\mathbb{A}^{\infty, o}\right)$. Nous fixons par la suite un isomorphisme de groupes $\bar{D}^{*}\left(\mathbb{A}^{\infty, o}\right) \simeq D^{*}\left(\mathbb{A}^{\infty, o}\right)$, obtenu à partir de l'isomorphisme précédent entre les algèbres $\bar{D}\left(\mathbb{A}^{\infty, o}\right)$ et $D\left(\mathbb{A}^{\infty, o}\right)$. Soit également un isomorphisme $\bar{D}^{*}\left(F_{o}\right) \simeq \mathrm{GL}_{d}\left(F_{o}\right)$, que l'on fixe.

Enfin, notons $\mathcal{O}^{\infty, o}=\prod_{x \neq \infty, o} \mathcal{O}_{x} \subset \mathbb{A}^{\infty, o}$ et $\mathcal{D}^{\infty, o}=\prod_{x \neq \infty, o} \mathcal{D}_{x} \subset D\left(\mathbb{A}^{\infty, o}\right) ;$ pour $I \subset X$ un sous-schéma fini fermé non vide tel que $I \cap\{\infty, o\}=\emptyset$ et $\mathfrak{I} \subset \mathcal{O}_{X}$ le faisceau d'idéaux 
correspondant, on définit

$$
K_{I}^{\infty, o}=\operatorname{Ker}\left[\left(\mathcal{D}^{\infty, o}\right)^{*} \longrightarrow\left(\mathfrak{I}^{\infty, o} \mathcal{D}^{\infty, o} \backslash \mathcal{D}^{\infty, o}\right)^{*}\right] .
$$

Le groupe $K_{I}^{\infty, o}$ est un sous-groupe ouvert compact de $D^{*}\left(\mathbb{A}^{\infty, o}\right)$. En particulier, via l'identification $\bar{D}^{*}\left(\mathbb{A}^{\infty, o}\right)=D^{*}\left(\mathbb{A}^{\infty, o}\right)$, on peut considérer $K_{I}^{\infty, o}$ comme un sous-groupe (ouvert compact) de $\bar{D}^{*}\left(\mathbb{A}^{\infty, o}\right)$. Pour finir, on introduit l'ensemble suivant de doubles classes, noté $Z_{I}$ :

$$
Z_{I}=\bar{D}^{*}(F) \backslash \bar{D}^{*}\left(\mathbb{A}^{\infty}\right) / K_{I}^{\infty, o}
$$

(où $\bar{D}^{*}(F)$ agit à gauche sur $\bar{D}^{*}\left(\mathbb{A}^{\infty}\right)$ et $K_{I}^{\infty, o}$ à droite). Cet ensemble est muni d'une action à droite évidente du groupe $\bar{D}^{*}\left(F_{o}\right)=\mathrm{GL}_{d}\left(F_{o}\right)$, et le quotient par cette action est fini.

Pour $I \hookrightarrow J$ deux sous-schémas fermés finis emboîtés de $X \backslash\{\infty, o\}$, l'inclusion $K_{J}^{\infty, o} \subset K_{I}^{\infty, o}$ induit un morphisme $Z_{J} \rightarrow Z_{I}$. Les ensembles $Z_{I}$ constituent donc pour $I$ de plus en plus grand un système projectif où opère le groupe $\bar{D}^{*}\left(\mathbb{A}^{\infty, o}\right)$.

8.2. Le théorème d'uniformisation. - A partir de maintenant, seul nous importe le problème de modules au-dessus de $\mathcal{O}_{o}$; autrement dit, on regarde $\mathcal{E} \ell_{X, \mathcal{D}, I}$ comme un schéma sur $\operatorname{Spec} \mathcal{O}_{o}$.

Théorème 8.1. - Rappelons que $I \cap\{\infty, o\}=\emptyset$. Avec les conventions et notations précédentes, on a un isomorphisme de $\mathcal{O}_{o}$-schémas formels :

$$
\widehat{\mathcal{E} \ell \ell}_{X, \mathcal{D}, I} \simeq\left[\left(\widehat{\Omega}^{d} \hat{\otimes}_{\mathcal{O}_{o}} \widehat{\mathcal{O}}_{o}^{n r}\right) \times Z_{I}\right] / \mathrm{GL}_{d}\left(F_{o}\right)
$$

où $\widehat{\mathcal{E}}_{X, \mathcal{D}, I}$ désigne le complété formel de $\mathcal{E} \ell_{X, \mathcal{D}, I}$ le long de sa fibre spéciale. Ces isomorphismes sont compatibles, lorsque I varie, avec les morphismes de restriction du niveau. L'isomorphisme des deux systèmes projectifs ainsi obtenu est compatible à l'action sur les deux membres du groupe $D^{*}\left(\mathbb{A}^{\infty, o}\right) \simeq \bar{D}^{*}\left(\mathbb{A}^{\infty, o}\right)$. Enfin, ces isomorphismes se relèvent en des isomorphismes entre les $\mathcal{D}_{o}$-modules formels spéciaux naturellement portés par les deux membres.

Remarque 8.2. - L'isomorphisme du théorème peut s'exprimer comme une uniformisation rigide-analytique :

$$
\left(\mathcal{E} \ell \ell_{X, \mathcal{D}, I}\right)^{a n} \simeq\left[\left(\Omega^{d} \hat{\otimes}_{F_{o}} \widehat{F}_{o}^{n r}\right) \times Z_{I}\right] / \mathrm{GL}_{d}\left(F_{o}\right)
$$

où $\left(\mathcal{E} \ell \ell_{X, \mathcal{D}, I}\right)^{a n}$ désigne l'espace rigide-analytique sur $F_{O}$ sous-jacent à $\mathcal{E} \ell_{X, \mathcal{D}, I}$ (c.-à-d. la fibre générique de $\widehat{\mathcal{E} \ell \ell}_{X, \mathcal{D}, I}$, au sens de Raynaud [Ra2]).

Donnons quelques précisions sur l'énoncé du théorème :

1. Rappelons que l'action de $\mathrm{GL}_{d}\left(F_{o}\right)$ sur $\widehat{\Omega}^{d} \hat{\otimes}_{\mathcal{O}_{o}} \widehat{\mathcal{O}}_{o}^{n r}$ considérée par Drinfeld (cf. 7.3) est obtenue à partir de l'action naturelle sur $\widehat{\Omega}^{d}$ et de l'action $g \mapsto \widetilde{F r}^{-v(\operatorname{det} g)}$ sur $\widehat{\mathcal{O}}_{o}^{n r}$; elle est définie seulement au-dessus de $\mathcal{O}_{o}$ et non pas de $\mathcal{O}_{o}^{n r}$. Ici, nous convertissons cette action à gauche en une action à droite, par composition avec la transposition $g \mapsto^{t} g$.

2. Le $\mathcal{D}_{o}$-module formel porté par $\widehat{\mathcal{E} \ell}_{X, \mathcal{D}, I}$ est le groupe formel $G r_{o}(\mathfrak{E})$ associé par la construction du paragraphe 2.2 au $\mathcal{D}$-faisceau elliptique universel $\mathfrak{E}$ donné par le problème de modules correspondant à $\mathcal{E} \ell_{X, \mathcal{D}, I}$. Celui porté par le membre de droite provient de la description modulaire de $\Omega^{d} \hat{\otimes}_{\mathcal{O}_{o}} \widehat{\mathcal{O}}_{o}^{n r}$ (cf. 7).

Pour finir, expliquons pourquoi le quotient $\left[\left(\widehat{\Omega}^{d} \hat{\otimes}_{\mathcal{O}_{o}} \widehat{\mathcal{O}}_{o}^{n r}\right) \times Z_{I}\right] / \mathrm{GL}_{d}\left(F_{o}\right)$ qui figure dans l'énoncé du théorème n'est rien d'autre qu'une réunion finie de formes tordues galoisiennes (sur des extensions non ramifiées) de quotients « à la Mumford» (cf. [M] ou [Ra1 dans le cas $d=2$ et $[\mathbf{M u}]$ en dimension supérieure), c'est-à-dire de la forme $\widehat{\Omega}^{d} / \Gamma$, pour $\Gamma \subset \mathrm{PGL}_{d}\left(F_{o}\right)$ des 
sous-groupes de congruence (précisément, des groupes de Schottky, lorsque $d=2$, au sens de [M] Def. 1.3; voir $\mathbf{M u} \S 1 \mathrm{C}$ pour leurs analogues en dimension supérieure).

Tout d'abord, l'action de $\bar{D}^{*}\left(F_{o}\right) \simeq \mathrm{GL}_{d}\left(F_{o}\right)$ sur $Z_{I}$ décompose ce dernier ensemble en un nombre fini d'orbites contenant chacune la double classe d'un élément $x$ dont la $o$-composante $x_{o}$ est égale à 1 ; le stabilisateur de $x$ est alors $\Gamma_{x}=\bar{D}^{*}(F) \cap x K_{I}^{\infty, o} x^{-1}$, où l'intersection est prise dans $\bar{D}^{*}\left(\mathbb{A}^{\infty, o}\right)$ puis, vue comme sous-groupe de $\bar{D}^{*}(F)$, injectée dans $\bar{D}^{*}\left(F_{o}\right)$. Ainsi le quotient en question apparait-il comme la réunion d'un nombre fini de quotients de la forme $\left(\widehat{\Omega}^{d} \hat{\otimes}_{\mathcal{O}_{o}} \widehat{\mathcal{O}}_{o}^{n r}\right) / \Gamma_{x_{i}}$, où les $\Gamma_{x_{i}}$ sont les différents stabilisateurs décrits précédemment (ce sont des sous-groupes discrets et co-compacts dans $\bar{D}^{*}\left(F_{o}\right)$ ). Par ailleurs, on vérifie sans mal que ces stabilisateurs contiennent chacun une puissance $\pi_{o}^{n_{i}} \operatorname{Id}_{d}$ de la matrice $\pi_{o} \operatorname{Id}_{d}$. On peut donc commencer par passer au quotient par l'action de $\pi_{o}^{n_{i}} \operatorname{Id}_{d}$ (qui agit trivialement sur $\widehat{\Omega}^{d}$ ); nos quotients s'écrivent alors $\left(\widehat{\Omega}^{d} \hat{\otimes}_{\mathcal{O}_{o}} \mathcal{O}_{o}^{\left(d n_{i}\right)}\right) / \Gamma_{x_{i}}$, où $\mathcal{O}_{o}^{\left(d n_{i}\right)}$ désigne l'extension non ramifiée de degré $d n_{i}$ de $\mathcal{O}_{o}$. Après extension des scalaires à $\mathcal{O}_{o}^{\left(d n_{i}\right)}$, cela devient isomorphe à une réunion finie de quotients de la forme $\widehat{\Omega}^{d} / \Gamma_{i}$, où $\Gamma_{i}$ désigne l'image dans $\mathrm{PGL}_{d}\left(F_{o}\right)$ du sous-groupe de $\Gamma_{x_{i}}$ constitué des éléments dont le déterminant est une unité. Ces derniers sont des quotients de Mumford (généralisés).

8.3. Généralisation : cas d'une structure de niveau en $o$. - On met maintenant une structure de niveau en la place $o: I$ désigne donc un sous-schéma fermé fini de $X-\{\infty\}$ tel que la multiplicité de $o$ dans $I$ soit un entier $n>0$. Cependant, munir un $\mathcal{D}$-faisceau elliptique $\left(\mathcal{E}_{i}, j, t\right)$, de zéro $z$, d'une structure de niveau $I$ n'a de sens que si $z(S)$ est disjoint de $V(I)$; le schéma $\mathcal{E} \ell_{X, \mathcal{D}, I}$ n'existe donc qu'en fibre générique.

Mais l'espace analytique $\left(\mathcal{E} \ell_{X, \mathcal{D}, I}\right)^{a n}$ sur $F_{o}$ sous-jacent à $\mathcal{E} l l_{X, \mathcal{D}, I}$ est bien défini (bien que l'on sache abstraitement que $\left(\mathcal{E} \ell_{X, \mathcal{D}, I}\right)^{a n}$ doit provenir d'un certain schéma formel, ce dernier n'est pas construit à priori, comme pour les revêtements $\left.\Sigma_{n}^{d}\right)$. Comparativement au théorème 8.1, on obtient également une uniformisation rigide-analytique de nos schémas de modules $\mathcal{E} \ell_{X, \mathcal{D}, I}$, mais en termes des revêtements $\Sigma_{n}^{d}$ de $\Omega^{d} \hat{\otimes}_{F_{o}} \widehat{F}_{o}^{n r}$ définis au paragraphe 7.4 (regardés au-dessus de $F_{o}$ par restriction des scalaires) :

Théorème 8.3. - Il existe un isomorphisme d'espaces rigides-analytiques sur $F_{o}$ :

$$
\left(\mathcal{E} \ell \ell_{X, \mathcal{D}, I}\right)^{a n} \simeq\left[\Sigma_{n}^{d} \times Z_{I^{o}}\right] / \mathrm{GL}_{d}\left(F_{o}\right) .
$$

Ces isomorphismes sont compatibles, lorsque I varie (i.e. $n$ et $I^{o}$ varient), aux opérations de projection, et l'isomorphisme ainsi obtenu entre les deux systèmes projectifs est équivariant pour l'action du groupe $D^{*}\left(\mathbb{A}^{\infty}\right) \simeq \bar{D}^{*}\left(\mathbb{A}^{\infty, o}\right) \times D_{o}^{*}$.

(l'action de $D_{o}^{*}$ sur les revêtements $\Sigma_{n}^{d}$ dont il est question ici est l'action à gauche considérée par Drinfeld (cf. [7.3), transformée en une action à droite via $g \mapsto g^{-1}$ )

Remarque. - Comme précédemment, le quotient du membre de droite de la formule n'est rien d'autre qu'une réunion finie de formes tordues galoisiennes de quotients $\Sigma_{n}^{d} / \Gamma_{i}$, pour des sous-groupes de congruences $\Gamma_{i} \subset \mathrm{PGL}_{d}\left(F_{o}\right)$.

Nous allons voir que le théorème 8.3 est en fait une conséquence « formelle » du théorème 8.1. Notons $\mathfrak{E}$ le $\mathcal{D}$-faisceau elliptique « universel » sur le $\mathcal{O}_{o^{-}}$schéma $\mathcal{E} l_{X, \mathcal{D}, I^{\circ}}$ défini par le problème de modules correspondant à $\mathcal{E} \ell_{X, \mathcal{D}, I^{o}}$. D'après la proposition 2.7, $\mathcal{E} \ell_{X, \mathcal{D}, I}$, vu comme

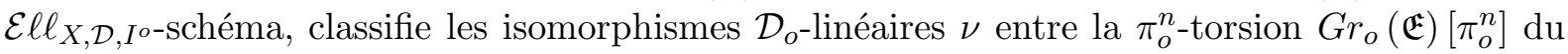


groupe $G r_{o}(\mathfrak{E})$ et $\mathcal{D}_{o} \otimes_{\mathcal{O}_{o}}\left(\mathcal{O}_{o} / \pi_{o}^{n}\right)$. La donnée d'un tel isomorphisme revient encore à la donnée d'un point exactement d'ordre $q^{d^{2} n}$ dans $G r_{o}(\mathfrak{E})\left[\pi_{o}^{n}\right]$.

Par ailleurs, il existe, d'après la dernière assertion du théorème 8.1, un isomorphisme de $\mathcal{D}_{o^{-}}$-modules formels sur $\widehat{\mathcal{E} \ell \ell}_{X, \mathcal{D}, I^{\circ}}$ (avec les notations et définitions du paragraphe 7.4 ) :

$$
G r_{o}(\mathfrak{E}) \simeq\left[Y \times Z_{I^{o}}\right] / \mathrm{GL}_{d}\left(F_{o}\right),
$$

et donc également au niveau de la $\pi_{o}^{n}$-torsion :

$$
G r_{o}(\mathfrak{E})\left[\pi_{o}^{n}\right] \simeq\left[Y_{n}^{d} \times Z_{I^{o}}\right] / \mathrm{GL}_{d}\left(F_{o}\right)
$$

d'où un isomorphisme au-dessus de $\left(\mathcal{E} \ell_{X, \mathcal{D}, I^{\circ}}\right)^{a n}$ :

$$
\left(G r_{o}(\mathfrak{E})\left[\pi_{o}^{n}\right]\right)^{a n} \simeq\left[\mathcal{Y}_{n}^{d} \times Z_{I^{o}}\right] / \operatorname{GL}_{d}\left(F_{o}\right)
$$

Finalement, on a donc bien :

$$
\left(\mathcal{E} \ell_{X, \mathcal{D}, I}\right)^{a n} \simeq\left[\Sigma_{n}^{d} \times Z_{I^{o}}\right] / \mathrm{GL}_{d}\left(F_{o}\right) .
$$

\subsection{Preuve du théorème 8.1 .}

8.4.1. Notations. - Fixons un $\mathcal{D}$-faisceau elliptique spécial $\mathcal{E}_{\Phi}$ de caractéristique $o$ sur $S=$ Spec $\overline{\kappa(o)}$, de $\mathcal{D}_{o}$-module formel associé $\Phi$ ( $\Phi$ est donc spécial). Puisque le schéma $\mathcal{E} \ell_{X, \mathcal{D}, I}$ est propre, sa fibre spéciale est donc non vide et un tel $\mathcal{E}_{\Phi}$ existe bien. Choisissons d'autre part une identification $\bar{D}=$ End $\left(V\left(\mathcal{E}_{\Phi}\right), \varphi_{\Phi}, \lambda_{\Phi}\right)$. Cela induit une identification

$$
\bar{D}^{*}\left(F_{o}\right)=\mathrm{GL}_{d}\left(F_{o}\right)=\operatorname{Aut}\left(V_{o}\left(\mathcal{E}_{\Phi}\right), \varphi_{\Phi, o}, \lambda_{\Phi, o}\right),
$$

et donc, via l'anti-équivalence de catégories entre $\mathcal{D}_{o}$-modules de coordonnées et $\mathcal{D}_{o}$-modules formels, une identification

$$
\mathrm{GL}_{d}\left(F_{o}\right)=\operatorname{Aut}_{\mathcal{D}_{o}}^{0}(\Phi)
$$

telle que le diagramme suivant soit commutatif :

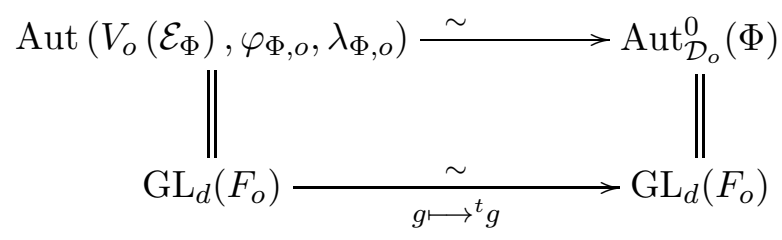

(les flèches horizontales sont des anti-isomorphismes).

Nous fixons pour finir un isomorphisme $D\left(\mathbb{A}^{\infty, o}\right)$-équivariant :

$$
\nu_{\Phi}=\left(\nu_{\Phi, x}\right): V^{\infty, o} \stackrel{\sim}{\longrightarrow}\left(V^{\infty, o}\left(\mathcal{E}_{\Phi}\right)\right)^{\varphi_{\Phi}^{\infty, o}}
$$

compatible à l'isomorphisme fixé entre $\bar{D}\left(\mathbb{A}^{\infty, o}\right)$ et $D\left(\mathbb{A}^{\infty, o}\right)$ (cf. 8.1) : cela signifie que, via $\nu_{\Phi, x}(x \neq o, \infty)$, l'action de $\bar{D}=\operatorname{End}\left(V\left(\mathcal{E}_{\Phi}\right), \varphi_{\Phi}, \lambda_{\Phi}\right)$ induite sur $V_{x}$ est donnée par le composé :

$$
\bar{D} \hookrightarrow \bar{D}\left(F_{x}\right) \simeq D\left(F_{x}\right) \stackrel{\sim}{\longrightarrow} \operatorname{End}_{D_{x}}\left(V_{x}\right)
$$

(action par multiplication à droite). 
8.4.2. Algébrisations. - Soit $S$ un $\mathcal{O}_{o^{-}}$-schéma où l'image de $\pi_{o}$ est nilpotente et $X$ un $\mathcal{D}_{o^{-}}$ module formel spécial sur $S$. On note $z: S \rightarrow \operatorname{Spec} \mathcal{O}_{o}$ le morphisme structural.

Définition 8.4. - Une algébrisation de $X$ est la donnée d'un couple $(\mathcal{E}, \varepsilon)$ constitué d'un $\mathcal{D}$ -

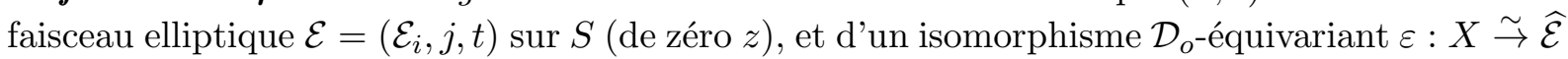
entre $X$ et le groupe formel $\widehat{\mathcal{E}}=G r_{o}(\mathcal{E})$ associé à $\mathcal{E}(\mathcal{E}$ est donc spécial). Lorsque $\mathcal{E}$ est de plus muni d'une structure de niveau $I$, on parle d'algébrisation avec structure de niveau $I$.

A partir de maintenant, nous supposerons que $S=\operatorname{Spec} \overline{\kappa(o)}$ et que $z$ est le morphisme $S \rightarrow \operatorname{Spec} \kappa(o) \hookrightarrow \operatorname{Spec} \mathcal{O}_{o}$. Dans ce cas, nous allons voir que l'ensemble $\mathcal{A l g} g_{I}(\Phi)$ des classes d'isomorphie d'algébrisations avec structure de niveau $I$ de $\Phi$ est en fait en bijection naturelle avec l'ensemble de doubles classes $Z_{I}$ défini en 8.1 .

Commençons par « adèliser» la situation. Pour $I \subset X$ un sous-schéma fini fermé non vide de $X \backslash\{\infty, o\}$ et $\mathfrak{I}$ le faisceau d'idéaux correspondant, une structure $\iota$ de niveau $I$ sur $\left(\mathcal{E}_{i}, j, t\right)$ est équivalente à la donnée pour tout $x \neq \infty$,o d'un morphisme $\left(\Im_{x} \mathcal{D}_{x} \backslash \mathcal{D}_{x}\right)$-équivariant

$$
\iota_{x}:\left(\mathfrak{I}_{x} \mathcal{D}_{x} \backslash \mathcal{D}_{x}\right) \otimes \overline{\kappa(o)} \stackrel{\sim}{\longrightarrow} \mathfrak{I}_{x} \mathcal{E}_{x} \backslash \mathcal{E}_{x}
$$

rendant commutatif le diagramme :

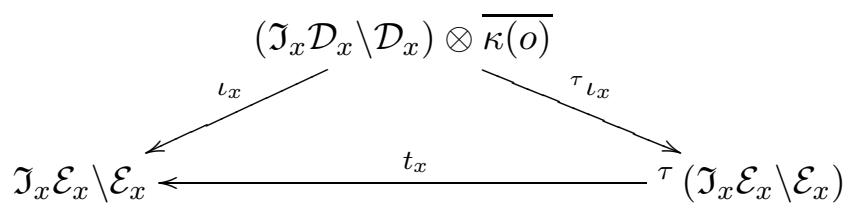

On peut donc également voir $\iota$ comme une classe $\bar{\iota}$ modulo $K_{I}^{\infty, o}$ d'isomorphismes $\mathcal{D}^{\infty, o}$-équivariants $\iota: \mathcal{D}^{\infty, o} \otimes \overline{\kappa(o)} \stackrel{\sim}{\longrightarrow} \mathcal{E} \otimes \mathcal{O}^{\infty, o}$ tels que l'action de $t^{\infty, o}=\prod_{x \neq \infty, o} t_{x}$ sur le membre de droite corresponde à celle de $\operatorname{Id}_{\mathcal{D}^{\infty}, o} \otimes F r$ sur celui de gauche (avec les notations de 8.1). En définitive, $\mathcal{A} \operatorname{Ag}_{I}(\Phi)$ est l'ensemble des classes d'isomorphie de triplets $(\mathcal{E}, \varepsilon, \bar{\iota})$ où $\mathcal{E}$ est un $\mathcal{D}$-faisceau elliptique sur $\overline{\kappa(o)}$, où $\varepsilon$ est un isomorphisme $\mathcal{D}_{o}$-équivariant entre $\Phi$ et $\widehat{\mathcal{E}}$, et $\bar{\iota}$ une classe modulo $K_{I}^{\infty, o}$ de tels isomorphismes $\iota$.

La description suivante s'avère plus commode pour ce qui suit : on peut voir encore $\mathcal{A} l g_{I}(\Phi)$ comme l'ensemble des classes d'isogénie de triplets $(\mathcal{E}, \varepsilon, \bar{\nu})$ où $\mathcal{E}$ est un $\mathcal{D}$-faisceau elliptique sur $\overline{\kappa(o)}$, où $\varepsilon$ est une quasi-isogénie (équivariante) entre $\Phi$ et $\widehat{\mathcal{E}}$, et où enfin $\bar{\nu}$ est une classe modulo $K_{I}^{\infty, o}$ d'isomorphismes $D\left(\mathbb{A}^{\infty, o}\right)$-équivariants :

$$
\nu:\left(V^{\infty, o} \hat{\otimes} \overline{\kappa(o)}, \operatorname{Id}_{V^{\infty, o}} \hat{\otimes} F r\right) \stackrel{\sim}{\longrightarrow}\left(V^{\infty, o}(\mathcal{E}), \varphi^{\infty, o}\right),
$$

lesquels sont encore équivalents à la donnée d'isomorphismes $D\left(\mathbb{A}^{\infty, o}\right)$-équivariants :

$$
\nu=\left(\nu_{x}\right): V^{\infty, o} \stackrel{\sim}{\longrightarrow}\left(V^{\infty, o}(\mathcal{E})\right)^{\varphi^{\infty, o}} .
$$

Ce raisonnement est standard : essentiellement, c'est le fait bien connu qu'un fibré vectoriel $\mathcal{L}$ sur $X$ est déterminé par sa fibre générique $\mathcal{L}_{\eta}$ et la donnée des $\mathcal{O}_{v}$-réseaux $\mathcal{L}_{v}$ en toute place $v$ de $X$. En résumé, $\mathcal{A l g}_{I}(\Phi)$ est l'ensemble des classes d'équivalence de triplets $(\mathcal{E}, \varepsilon, \bar{\nu})$, où $(\mathcal{E}, \varepsilon, \bar{\nu}) \sim$ $\left(\mathcal{E}^{\prime}, \varepsilon^{\prime}, \bar{\nu}^{\prime}\right)$ si et seulement si il existe une isogénie $\alpha=\left(\alpha_{x}\right):(V(\mathcal{E}), \varphi, \lambda) \stackrel{\sim}{\rightarrow}\left(V\left(\mathcal{E}^{\prime}\right), \varphi^{\prime}, \lambda^{\prime}\right)$ rendant commutatifs les diagrammes suivants :
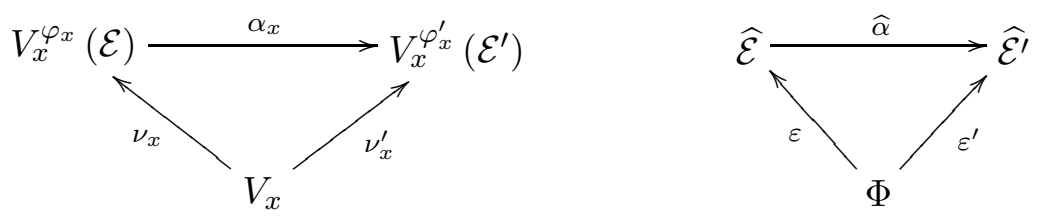
où $\widehat{\alpha}$ désigne la quasi-isogénie $\left(D_{o}\right.$-linéaire) induite (via l'anti-équivalence de catégories entre $\mathcal{D}_{o^{-}}$ modules de coordonnées et $\mathcal{D}_{o^{-}}$-modules formels $)$par $\alpha_{o}^{-1}:\left(V_{o}\left(\mathcal{E}^{\prime}\right), \varphi_{o}^{\prime}, \lambda_{o}^{\prime}\right) \stackrel{\sim}{\rightarrow}\left(V_{o}(\mathcal{E}), \varphi_{o}, \lambda_{o}\right)$.

Soit $\mathcal{A} l g_{\infty}(\Phi)$ la limite projective selon $I \not \supset \infty \operatorname{des} \mathcal{A} l g_{I}(\Phi)$; cet ensemble s'identifie à l'ensemble des (classes d'équivalence de) tels triplets $(\mathcal{E}, \varepsilon, \nu)$. Cette description met en évidence une action à droite sur $\mathcal{A} l g_{\infty}(\Phi)$ du groupe $\bar{D}^{*}\left(\mathbb{A}^{\infty}\right)=\bar{D}^{*}\left(F_{o}\right) \times \bar{D}^{*}\left(\mathbb{A}^{\infty, o}\right)$ : la composante suivant $\bar{D}^{*}\left(F_{o}\right)$ agit par composition sur $\varepsilon$ et celle suivant $\bar{D}^{*}\left(\mathbb{A}^{\infty, o}\right)$ par composition sur $\nu$. Noter cependant, en vertu des identifications faites au paragraphe 8.4.1, qu'un élément $g$ de $\mathrm{GL}_{d}\left(F_{o}\right)$ compose $\varepsilon$ à droite $\operatorname{par}^{t} g^{-1} \in \operatorname{Aut}_{\mathcal{D}_{o}}^{0}(\Phi)$.

Cette action est transitive, ainsi qu'il résulte de l'unicité de la classe d'isogénie de $\mathcal{E}$ (c'est un $\mathcal{D}$-faisceau elliptique spécial sur $\overline{\kappa(o)}$; voir $[5.2)$. En effet, utilisant les identifications de 8.4.1, on considère l'élément de $\mathcal{A} l g_{\infty}(\Phi)$ donné par le triplet $\left(\mathcal{E}_{\Phi}, \varepsilon_{\Phi}: \widehat{\mathcal{E}_{\Phi}}=\Phi, \nu_{\Phi}=\prod_{x \neq \infty, o} \nu_{\Phi, x}\right)$. Si $\alpha:(V(\mathcal{E}), \varphi, \lambda) \stackrel{\sim}{\rightarrow}\left(V\left(\mathcal{E}_{\Phi}\right), \varphi_{\Phi}, \lambda_{\Phi}\right)$ désigne un isomorphisme entre fibres génériques, on voit alors que $(\mathcal{E}, \varepsilon, \nu)$ est l'image de $\left(\mathcal{E}_{\Phi}, \varepsilon_{\Phi}, \nu_{\Phi}\right)$ par l'élément $\gamma=\left(\gamma_{x}\right)$ de $\bar{D}^{*}\left(\mathbb{A}^{\infty}\right)$ défini par les diagrammes commutatifs suivants :
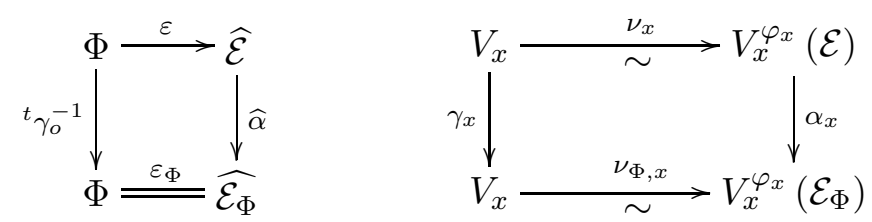

D'autre part, le stabilisateur de ce triplet de base $\left(\mathcal{E}_{\Phi}, \varepsilon_{\Phi}, \nu_{\Phi}\right)$ est le sous-groupe $\bar{D}^{*}(F) \subset$ $\bar{D}^{*}\left(\mathbb{A}^{\infty}\right)$. Cela ressort de la commutativité des diagrammes ci-dessous, où $\gamma$ désigne un élément de $\bar{D}^{*}(F)$ et $\gamma_{x}$ ses images dans $\bar{D}^{*}\left(F_{x}\right)$ :
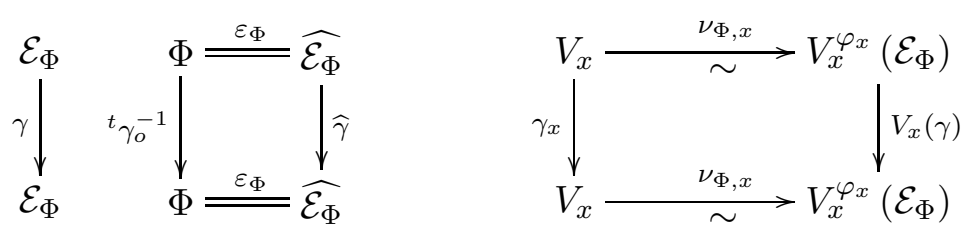

On en déduit une bijection entre $\mathcal{A l} g_{\infty}(\Phi)$ et l'espace homogène $\bar{D}^{*}(F) \backslash \bar{D}^{*}\left(\mathbb{A}^{\infty}\right)$, d'où résulte la bijection annoncée :

$$
\mathcal{A} \operatorname{Alg}_{I}(\Phi) \simeq \bar{D}^{*}(F) \backslash \bar{D}^{*}\left(\mathbb{A}^{\infty}\right) / K_{I}^{\infty, o}=Z_{I}
$$

8.4.3. Construction du morphisme $\bar{\Theta}$. - Le but de ce paragraphe est de définir un morphisme $\Theta$ de la fibre spéciale $\left[\left(\widehat{\Omega}^{d} \hat{\otimes}_{\mathcal{O}_{o}} \widehat{\mathcal{O}}_{o}^{n r}\right) \otimes \kappa(o)\right] \times Z_{I}=\left(\widehat{\Omega}^{d} \otimes \overline{\kappa(o)}\right) \times Z_{I}$ vers la fibre spéciale $\mathcal{E} \ell_{X, \mathcal{D}, I} \otimes \kappa(o)$, puis de vérifier qu'il se factorise à travers l'action de $\mathrm{GL}_{d}\left(F_{o}\right)$, en un morphisme $\bar{\Theta}$.

Soit donc $S$ un $\kappa(o)$-schéma. Si $\mathcal{E}$ et $\mathcal{E}^{\prime}$ sont deux $\mathcal{D}$-faisceaux elliptiques sur $S$, nous appellerons «o-isogénie » $\mathcal{E} \rightarrow \mathcal{E}^{\prime}$ une isogénie $g:(V(\mathcal{E}), \varphi, \lambda) \stackrel{\sim}{\rightarrow}\left(V\left(\mathcal{E}^{\prime}\right), \varphi^{\prime}, \lambda^{\prime}\right)$ qui, en toute place $x \neq o, \infty$, induit (par tensorisation $\otimes_{F} F_{x}$ ) un isomorphisme $D_{x}$-linéaire $g_{x}:\left(V_{x}(\mathcal{E}), \varphi_{x}\right) \stackrel{\sim}{\rightarrow}$ $\left(V_{x}\left(\mathcal{E}^{\prime}\right), \varphi_{x}^{\prime}\right)$ entre $F_{x}$-modules de Dieudonné sur $S$, ce dernier provenant (par tensorisation $\left.\otimes_{\mathcal{O}_{x}} F_{x}\right)$ d'un isomorphisme $\mathcal{D}_{x}$-linéaire $\tilde{g}_{x}: \mathcal{E}_{x} \stackrel{\sim}{\rightarrow} \mathcal{E}_{x}^{\prime}$ faisant commuter le diagramme :

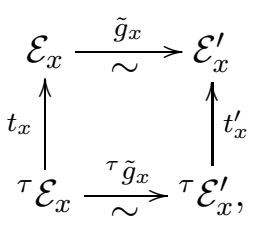


et enfin, tel que, en $x=\infty, g_{\infty}$ provient d'une série d'isomorphismes $\tilde{g}_{\infty, i}:\left(\mathcal{E}_{i}\right)_{\infty} \stackrel{\sim}{\rightarrow}\left(\mathcal{E}_{i}^{\prime}\right)_{\infty}$ faisant commuter tous les diagrammes évidents.

Le lemme fondamental est le suivant :

Lemme 8.5. - Soient $H_{1}$ et $H_{2}$ deux $\mathcal{D}_{o}$-modules formels spéciaux sur $S$, et $f: H_{1} \rightarrow H_{2}$ une quasi-isogénie. Soit d'autre part $\left(\mathcal{E}_{1}, \varepsilon_{1}\right)$ une algébrisation de $H_{1}$. Il existe alors une algébrisation $\left(\mathcal{E}_{2}, \varepsilon_{2}\right)$ de $H_{2}$, et une o-isogénie $h: \mathcal{E}_{1} \rightarrow \mathcal{E}_{2}$ telles que le diagramme suivant soit commutatif :

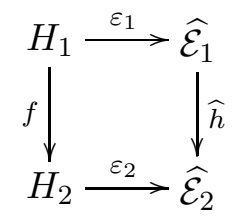

où $\widehat{h}$ désigne la quasi-isogénie ( $D_{o}$-linéaire) induite par l'isomorphisme $h_{o}^{-1}:\left(V_{o}\left(\mathcal{E}_{2}\right), \varphi_{2, o}, \lambda_{2, o}\right)$ $\stackrel{\sim}{\rightarrow}\left(V_{o}\left(\mathcal{E}_{1}\right), \varphi_{1, o}, \lambda_{1, o}\right)$. Le triplet $\left(\mathcal{E}_{2}, \varepsilon_{2}, h\right)$ est uniquement déterminé à isomorphisme près par cette propriété. Si de plus $\mathcal{E}_{1}$ est muni d'une structure de niveau I, alors (via h) il en est de même de $\mathcal{E}_{2}$.

Démonstration. - Soit $M$ le $\mathcal{D}_{0}$-module de coordonnées associé à $H_{2}$. Les données sont les suivantes : la fibre générique $\left(\mathcal{E}_{1}\right)_{\eta}$, un isomorphisme $\left(\mathcal{E}_{1}\right)_{x} \stackrel{\sim}{\rightarrow}\left(\mathcal{E}_{2}\right)_{x}$ pour tout $x \neq o, \infty$, en $x=\infty$ des isomorphismes $\left(\mathcal{E}_{1, i}\right)_{\infty} \stackrel{\sim}{\rightarrow}\left(\mathcal{E}_{2, i}\right)_{\infty}$, et en $x=o$ un isomorphisme $\left(\mathcal{E}_{1}\right)_{o} \otimes_{\mathcal{O}_{o}} F_{o} \underset{\alpha}{\stackrel{\sim}{\rightarrow}} M \otimes_{\mathcal{O}_{o}} F_{o}$. On voit facilement que le $\mathcal{D}$-faisceau elliptique $\mathcal{E}_{2}=\left(\mathcal{E}_{2, i}, j_{2}, t_{2}\right)$ défini comme suit convient : les sections locales $s$ de $\mathcal{E}_{2, i}$ sont les sections méromorphes de $\mathcal{E}_{1, i}$, régulières hors $o$, et en $o$ telles que $\alpha \circ s$ soit un élément du $\mathcal{O}_{o}$-module libre $M$. Le morphisme $t_{2}$ provient de $t_{1}$ et de $F$. L'unicité à isomorphisme près est claire également.

Utilisant le théorème de Drinfeld (théorème [7.4), ainsi que la bijection $\mathcal{A l g} g_{I}(\Phi) \simeq Z_{I}$ (cf. 8.4.2), on voit que se donner une section de $\left(\widehat{\Omega}^{d} \otimes \overline{\kappa(o)}\right) \times Z_{I}$ au-dessus d'un $\kappa(o)$-schéma (connexe) $S=\operatorname{Spec} B$ revient à se donner :

1. Un homomorphisme $\psi: \overline{\kappa(o)} \rightarrow B$;

2. Une classe d'isomorphie de couples $(H, \rho)$ avec:

- $H$ un $\mathcal{D}_{o}$-module formel spécial sur $S$;

- $\rho: \psi_{*} \Phi \rightarrow H$ une quasi-isogénie de hauteur 0 ;

3. Un triplet $(\mathcal{E}, \varepsilon, \bar{\nu}) \in \mathcal{A} \lg _{I}(\Phi)$.

Partant de ces données, on applique le lemme 8.5 ci-dessus avec $H_{1}=\psi_{*} \Phi, H_{2}=H, f=$ $\rho, \mathcal{E}_{1}=\psi_{*} \mathcal{E}, \varepsilon_{1}=\psi_{*} \varepsilon$; l'algébrisation $\left(\mathcal{E}_{2}, \epsilon_{2}, \bar{\nu}_{2}\right)$ de $H$ avec structure de niveau $I$ obtenue fournit alors un $\mathcal{D}$-faisceau elliptique $\mathcal{E}_{2}$ sur $B$, muni d'une $I$-structure de niveau. Cela définit un morphisme entre foncteurs, d'où résulte le morphisme de $\kappa(o)$-schémas :

$$
\Theta:\left(\widehat{\Omega}^{d} \otimes \overline{\kappa(o)}\right) \times Z_{I} \longrightarrow \mathcal{E} \ell \ell_{X, \mathcal{D}, I} \otimes \kappa(o) .
$$

Action de $\mathrm{GL}_{d}\left(F_{o}\right)$ : rappelons que, d'après 7.3 , l'action (convertie en une action à droite, cf. les commentaires suivant l'énoncé du théorème 8.1) d'un élément $g$ de $\mathrm{GL}_{d}\left(F_{o}\right)$ sur le foncteur $G$ est donnée par :

$$
(\psi, H, \rho) \cdot g=\left(\psi \circ \mathrm{Fr}^{-n}, H, \rho \circ \psi_{*}\left({ }^{t} g^{-1} \circ \mathrm{Frob}^{n}\right)\right),
$$

où l'on a posé $n=v(\operatorname{det} g)$. D'autre part, l'action sur $Z_{I}$ correspond, par la bijection équivariante du paragraphe 8.4.2, à une action sur $\mathcal{A} l g_{I}(\Phi)$ qui peut être décrite en terme du lemme 8.5 
comme suit : l'image $\left(\mathcal{E}_{1}, \varepsilon_{1}, \bar{\nu}_{1}\right)=(\mathcal{E}, \varepsilon, \bar{\nu}) \cdot g$ est caractérisée par l'existence d'une $o$-isogénie $h_{g}: \mathcal{E} \rightarrow \mathcal{E}_{1}$ rendant commutatif le diagramme suivant :

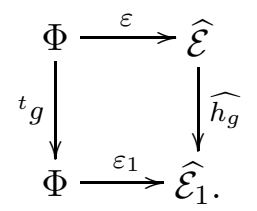

Notons $\left(\mathcal{E}_{2}, \varepsilon_{2}, \bar{\nu}_{2}\right)$ l'algébrisation de $H$ associée (par la construction précédente définissant $\Theta$ ) au point $x$ défini par $(\psi, H, \rho, \mathcal{E}, \varepsilon, \bar{\nu})$; on a donc un diagramme commutatif :

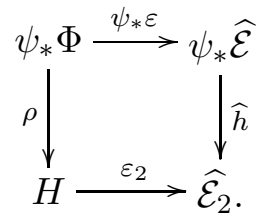

On vérifie alors que le point $x \cdot g$ défini par $\left(\psi_{1}=\psi \circ F r^{-n}, H, \rho_{1}=\rho \circ \psi_{*}\left({ }^{t} g^{-1} \circ \mathrm{Frob}^{n}\right), \mathcal{E}_{1}, \varepsilon_{1}, \bar{\nu}_{1}\right)$ a pour image la même algébrisation de $H$ : cela résulte de la commutativité du diagramme :

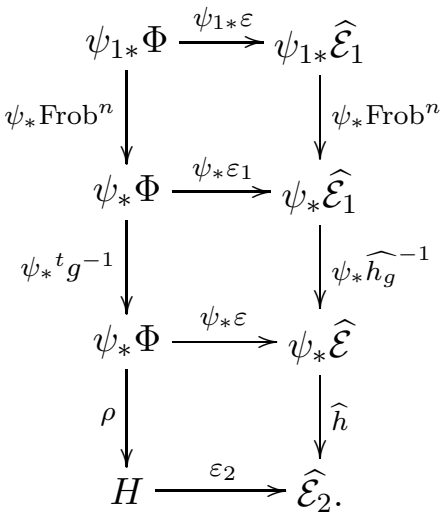

On voit donc que $\Theta$ se factorise en un morphisme de $\kappa(o)$-schémas :

$$
\bar{\Theta}:\left[\left(\widehat{\Omega}^{d} \otimes \overline{\kappa(o)}\right) \times Z_{I}\right] / \mathrm{GL}_{d}\left(F_{o}\right) \longrightarrow \mathcal{E} \ell \ell_{X, \mathcal{D}, I} \otimes \kappa(o) .
$$

8.4.4. $\bar{\Theta}$ est un isomorphisme. - Il est commode d'étendre les scalaires de $\kappa(o)$ à $\overline{\kappa(o)}$. Notons $\widehat{\Omega}^{d} \overline{\kappa(o)}$ le schéma déduit de $\widehat{\Omega}^{d}$ par extension des scalaires à $\overline{\kappa(o)}$. En termes modulaires, $\widehat{\Omega}^{d} \overline{\kappa(o)}$ représente le foncteur $\bar{G}$ qui classifie les couples $(H, \rho)$ (cf. paragraphe 17.2) ; le sous-groupe $\mathrm{GL}_{d}^{\prime}\left(F_{o}\right)$ de $\mathrm{GL}_{d}\left(F_{o}\right)$ constitué des $g$ tels que $v(\operatorname{det} g)=0$ y opère par composition sur $\rho$.

Le quotient $\left[\left(\widehat{\Omega}^{d} \otimes \overline{\kappa(o)}\right) \times Z_{I}\right] / \mathrm{GL}_{d}\left(F_{o}\right)$, après extension des scalaires, s'identifie au schéma $\left(\widehat{\Omega}_{\overline{\kappa(o)}}^{d} \times Z_{I}\right) / \mathrm{GL}_{d}^{\prime}\left(F_{o}\right)$. En effet, désignant par des indices différents les deux copies du corps $\overline{\kappa(o)}$, le schéma $\left[\left(\widehat{\Omega}^{d} \otimes \overline{\kappa(o)}_{(1)}\right) \otimes_{\kappa(o)} \overline{\kappa(o)}_{(2)}\right] \times Z_{I}$ obtenu par extension des scalaires se décompose en une somme de copies du schéma $\widehat{\Omega}^{d} \overline{\kappa(o)} \times Z_{I}$, indexées par le groupe de Galois $\operatorname{Gal}(\overline{\kappa(o)} / \kappa(o))=$

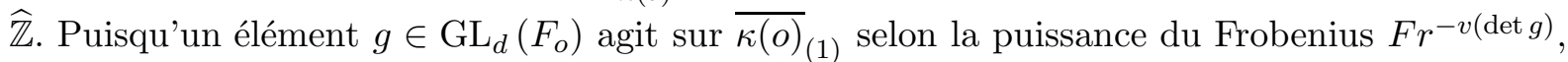
l'action du groupe $\mathrm{GL}_{d}\left(F_{o}\right)$ se traduit par une translation des indices. Cette action n'est pas transitive, mais le fait de quotienter d'abord par le centre $Z\left(\mathrm{GL}_{d}\left(F_{o}\right)\right)=d \mathbb{Z}$ du groupe donne lieu à une action transitive de $\mathrm{PGL}_{d}\left(F_{o}\right)$ sur le quotient $\bigoplus_{\sigma \in \mathbb{Z} / d \mathbb{Z}} \widehat{\Omega}^{d} \overline{\kappa(o)} \times Z_{I}$ obtenu. Cet espace homogène peut être décrit comme le quotient d'un élément par son stabilisateur, en l'occurrence $\left(\widehat{\Omega}^{d} \overline{\kappa(o)} \times Z_{I}\right) / \mathrm{GL}_{d}^{\prime}\left(F_{o}\right)$. 
Finalement, le morphisme $\bar{\Theta}_{\overline{\kappa(o)}}$ déduit de $\bar{\Theta}$ par extension des scalaires provient d'un morphisme de $\overline{\kappa(o)}$-schémas :

$$
\Theta_{1}: \widehat{\Omega}^{d} \overline{\kappa(o)} \times Z_{I} \longrightarrow \mathcal{E} \ell_{X, \mathcal{D}, I} \otimes \overline{\kappa(o)},
$$

lequel se factorise à travers l'action de $\mathrm{GL}_{d}^{\prime}\left(F_{o}\right)$. Ce morphisme $\Theta_{1}$ associe à un point $(H, \rho, \mathcal{E}, \varepsilon, \bar{\nu})$, défini sur une $\overline{\kappa(o)}$-algèbre $B$, l'algébrisation de $H$ obtenue par application du lemme 8.5 avec $H_{1}=\Phi_{B}, H_{2}=H, f=\rho, \mathcal{E}_{1}=\mathcal{E}_{B}, \varepsilon_{1}=\varepsilon_{B}$.

Montrons que $\bar{\Theta}_{\overline{\kappa(o)}}$ induit une bijection entre les ensembles de $\overline{\kappa(o)}$-points des deux schémas :

Injectivité : supposons que deux $\overline{\kappa(o)}$-points $x=(H, \rho, \mathcal{E}, \varepsilon, \bar{\nu})$ et $x^{\prime}=\left(H^{\prime}, \rho^{\prime}, \mathcal{E}^{\prime}, \varepsilon^{\prime}, \bar{\nu}^{\prime}\right)$ admettent par $\Theta_{1}$ la même image $\mathcal{E}_{2}$, un $\mathcal{D}$-faisceau elliptique spécial sur $\overline{\kappa(o)}$ muni d'une structure de niveau $I$. Parce que $\mathcal{E}_{2}$ est à la fois une algébrisation de $H$ et de $H^{\prime}$, on voit que $H$ s'identifie naturellement à $H^{\prime}$. Puis l'on constate, via cette identification et l'égalité $\operatorname{Aut}_{\mathcal{D}_{o}}^{0}(\Phi)=\mathrm{GL}_{d}\left(F_{o}\right)$, que $\rho$ et $\rho^{\prime}$ diffèrent par composition par un élément $g \in \mathrm{GL}_{d}^{\prime}\left(F_{o}\right)$ : en effet, la $\mathcal{O}_{o}$-hauteur de la quasi-isogénie $g=\rho^{\prime-1} \circ \rho$ est $d . v_{o}(\operatorname{det} g)=0$, puisque $\rho$ et $\rho^{\prime}$ sont de hauteur nulle. On vérifie alors que $\left(\mathcal{E}^{\prime}, \varepsilon^{\prime}, \bar{\nu}^{\prime}\right)=(\mathcal{E}, \varepsilon, \bar{\nu}) \cdot g$, ainsi qu'il résulte du diagramme commutatif :

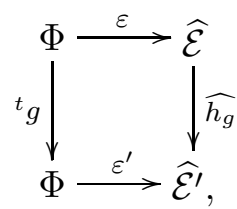

où $\widehat{h_{g}}$ provient de la $o$-isogénie $h_{g}=h^{\prime-1} \circ h: \mathcal{E} \rightarrow \mathcal{E}^{\prime}$, notant $h: \mathcal{E} \rightarrow \mathcal{E}_{2}\left(\right.$ resp. $h^{\prime}: \mathcal{E}^{\prime} \rightarrow \mathcal{E}_{2}$ ) la $o$-isogénie obtenue par application du lemme 8.5 lorsque l'on exprime que $\mathcal{E}_{2}$ est l'image par $\Theta_{1}$ du point $x$ (resp. $\left.x^{\prime}\right)$. On a donc $x^{\prime}=x \cdot g$, si bien que $x$ et $x^{\prime}$ coïncident modulo $\operatorname{GL}_{d}^{\prime}\left(F_{o}\right)$.

Surjectivité : Donnons-nous un $\mathcal{D}$-faisceau elliptique spécial $\mathcal{E}_{2}$ sur $\overline{\kappa(o)}$ et notons $H=\widehat{\mathcal{E}_{2}}$. Puisque tous les $\mathcal{D}_{o}$-modules formels spéciaux de hauteur $d^{2}$ sur $\overline{\kappa(o)}$ sont isogènes (cf. théorème 3.4), il existe une quasi-isogénie $\rho: \Phi \rightarrow H$. On peut même supposer, quitte à composer avec un endomorphisme convenable de $\Phi$, que $\rho$ est de hauteur 0. Appliquant le lemme 8.5 à l'algébrisation $\mathcal{E}_{2}$ de $H$ et à $\rho^{-1}$, on obtient alors une algébrisation $(\mathcal{E}, \varepsilon, \bar{\nu})$ de $\Phi$ avec structure de niveau $I$. Par construction même du morphisme $\Theta_{1}, \mathcal{E}_{2}$ admet $(H, \rho, \mathcal{E}, \varepsilon, \bar{\nu})$ pour antécédent.

Prouvons maintenant que $\Theta_{1}$ est étale, par le biais de l'étude des déformations. Soit donc $B$ une $\overline{\kappa(o)}$-algèbre et $B^{\prime} \rightarrow B$ un épaississement de $B$, de noyau un idéal de carré nul; on se donne un $B$-point $x=(H, \rho, \mathcal{E}, \varepsilon, \bar{\nu})$ de $\widehat{\Omega}^{d} \overline{\kappa(o)} \times Z_{I}$ et note $y=\mathcal{E}_{2}$ son image par $\Theta_{1}$. D'un côté, parce que la quasi-isogénie $\rho$ se déforme uniquement (cf. [Zi2 5.31) et que le schéma $Z_{I}$ est constant, déformer $x$ en un $B^{\prime}$-point revient à déformer le $\mathcal{D}_{o}$-module formel $H$. De l'autre côté, déformer $\mathcal{E}_{2}$ revient à déformer le $\mathcal{D}_{o}$-module formel $\widehat{\mathcal{E}}_{2}$ sous-jacent, en vertu (de l'analogue) du théorème de Serre et Tate (cf. théorème 4.1. A noter : au paragraphe 4 est étudiée la théorie absolue des déformations; lorsque $z: \mathcal{O}_{o} \rightarrow B$ est fixé, c'est-à-dire lorsque l'on regarde les déformations relatives, le fait que les déformations absolues se correspondent implique bien qu'il en est de même des déformations relatives). On voit donc que $\Theta_{1}$ met en bijection les déformations de $x$ et de $y$, puisque $\widehat{\mathcal{E}_{2}} \simeq H$ par construction de $\Theta_{1}$.

Par conséquent, $\bar{\Theta}_{\overline{\kappa(o)}}$ est étale; c'est donc un isomorphisme, puisqu'il est bijectif sur les $\overline{\kappa(o)}$-points. Finalement, $\bar{\Theta}$ est un isomorphisme.

8.4.5. Fin de la démonstration. - Il s'agit de prolonger en un isomorphisme

$$
\left[\left(\widehat{\Omega}^{d} \hat{\otimes} \widehat{\mathcal{O}}_{o}^{n r}\right) \times Z_{I}\right] / \mathrm{GL}_{d}\left(F_{o}\right) \stackrel{\sim}{\longrightarrow} \widehat{\mathcal{E} \ell \ell}_{X, \mathcal{D}, I}
$$


l'isomorphisme $\bar{\Theta}:\left[\left(\widehat{\Omega}^{d} \otimes \overline{\kappa(o)}\right) \times Z_{I}\right] / \mathrm{GL}_{d}\left(F_{o}\right) \stackrel{\sim}{\rightarrow} \mathcal{E} \ell \ell_{X, \mathcal{D}, I} \otimes \kappa(o)$ déjà construit entre les fibres spéciales des deux schémas formels. La possibilité de le faire va résulter du théorème de SerreTate et du fait, déjà constaté, que l'isomorphisme $\bar{\Theta}$ se relève aux $\mathcal{D}_{o}$-modules formels spéciaux naturellement portés par les deux membres.

Soit donc $B$ une $\mathcal{O}_{o}$-algèbre telle que l'image de $\pi_{o}$ soit nilpotente et $B_{0}=B / \pi_{o} B$. Comme précédemment, déformer de $B_{0}$ à $B$ un $B_{0}$-point $x_{0}$ du schéma $\left[\left(\widehat{\Omega}^{d} \hat{\otimes}_{\mathcal{O}_{o}^{n r}}^{n r} \times Z_{I}\right] / \mathrm{GL}_{d}\left(F_{o}\right)\right.$ revient à déformer le $\mathcal{D}_{o}$-module formel spécial $H_{0}$ issu de $x_{0}$ par la description modulaire de $\widehat{\Omega}^{d} \hat{\otimes} \widehat{\mathcal{O}}_{o}^{n r}$. Ainsi se donner un $B$-point $x$ du schéma quotient ci-dessus revient-il à se donner sa restriction $x_{0}$ à $B_{0}$, plus une déformation $H$ sur $B$ de $H_{0}$. De même, il est clair que se donner un $B$-point $y$ du schéma $\widehat{\mathcal{E} \ell}_{X, \mathcal{D}, I}$ revient à se donner sa restriction $y_{0}$ à $B_{0}$ et une déformation $\mathcal{E}$ sur $B$ du $\mathcal{D}$-faisceau elliptique spécial $\mathcal{E}_{0}$ sur $B_{0}$ défini par $y_{0}$. Or les déformations de $\mathcal{E}_{0}$ correspondent bijectivement, en vertu du théorème de Serre-Tate (cf. théorème 4.1), à celles de $\widehat{\mathcal{E}_{0}}$, lequel s'identifie à $H_{0}$. Cela prolonge de façon naturelle $\bar{\Theta}$ en un isomorphisme entre les schémas formels $\left[\left(\widehat{\Omega}^{d} \hat{\otimes} \widehat{\mathcal{O}}_{o}^{n r}\right) \times Z_{I}\right] / \mathrm{GL}_{d}\left(F_{o}\right)$ et $\widehat{\mathcal{E} \ell \ell_{X, \mathcal{D}, I}}$.

Pour finir, il est immédiat et formel de vérifier que ces isomorphismes sont compatibles entre eux lorsque $I$ varie et que le système projectif qu'ils constituent est $D^{*}\left(\mathbb{A}^{\infty, o}\right)$-équivariant. La preuve du théorème 8.1 est donc achevée.

\section{Troisième PARTIE III CONJECTURE DE DRINFELD-CARAYOL}

\section{Représentations cuspidales dans la cohomologie des revêtements $\Sigma_{n}^{d}$}

Soient $K$ un corps local non archimédien d'égale caractéristique $p, \mathcal{O}$ l'anneau des entiers, $\varpi$ une uniformisante et $\kappa$ le corps résiduel, de cardinal $q$. On choisit une clôture algébrique $\bar{K}$ de $K$ et note $K^{n r}$ l'extension maximale non-ramifiée de $K$ (dans $\bar{K}$ ); les anneaux des entiers respectifs sont $\overline{\mathcal{O}}$ et $\mathcal{O}^{n r}$, de corps résiduels $\bar{\kappa}$, une clôture algébrique de $\kappa$. Le groupe de Weil de $K$ est noté $W_{K}$; c'est le sous-groupe de $G_{K}=\operatorname{Gal}\left(K^{s} / K\right)$ constitué des éléments qui induisent sur $\bar{\kappa}$ une puissance entière du Frobenius ( $K^{s} \subset \bar{K}$ désigne une clôture séparable). Il est muni de la topologie pour laquelle le sous-groupe d'inertie $I_{K}$ de $\mathrm{Gal}\left(K^{s} / K\right)$, avec sa topologie de Krull, est un sous-groupe ouvert de $W_{K}$ et $W_{K} / I_{K} \simeq \mathbb{Z}$ est doté de la topologie discrète. On fixe également dans ce qui suit un nombre premier $\ell$ différent de $p$, ainsi qu'un isomorphisme $\overline{\mathbb{Q}}_{\ell} \simeq \mathbb{C}$.

\subsection{Les correspondances locales de Langlands et Jacquet-Langlands. -}

9.1.1. La correspondance de Jacquet-Langlands locale. - Soit $D_{d}$ 《l' » algèbre à division de centre $K$ et d'invariant $1 / d$. Désignons par $\mathcal{A}_{D_{d}}$ l'ensemble des (classes d'équivalence de) représentations complexes admissibles irréductibles du groupe $D_{d}^{*}$. D'autre part, soit $\mathcal{A}_{d}(K)$ l'ensemble des (classes d'équivalence de) représentations complexes admissibles irréductibles du groupe $\mathrm{GL}_{d}(K)$. On note $\mathcal{A}_{d}^{d}(K)$ le sous-ensemble constitué des représentations qui sont essentiellement de carré intégrable (i.e. les coefficients matriciels sont intégrables modulo le centre); parmi ces dernières figurent les représentations cuspidales.

Tant les éléments de $\mathcal{A}_{D_{d}}$ que ceux de $\mathcal{A}_{d}^{d}(K)$ admettent des caractères : c'est clair pour les premiers (ces représentations sont de dimension finie); pour les seconds, on définit d'abord un 
caractère distribution, dont on montre que sa restriction aux éléments réguliers semi-simples (i.e. dont le polynôme caractéristique est irréductible et à racines simples dans $\bar{K}$ ) est une fonction (voir $[\mathbf{L e}]$ ).

On dit que $\delta \in D_{d}^{*}$ et un élément semi-simple $g$ de $\mathrm{GL}_{d}(K)$ sont associés si le polynôme caractéristique réduit de $\delta$ coïncide avec le polynôme caractéristique de $g$ (dans ce cas, $g$ est automatiquement elliptique).

Théorème 9.1 (Badulescu, en égale caractéristique $p$ ). — Il existe une bijection

$$
\begin{aligned}
\mathcal{A}_{d}^{d}(K) & \longrightarrow \mathcal{A}_{D_{d}} \\
\pi & \longmapsto \mathrm{JL}(\pi)
\end{aligned}
$$

caractérisée par la propriété que les caractères de $\pi$ et $\mathrm{JL}(\pi)$ coïncident au signe $(-1)^{d-1}$ près sur les éléments associés réguliers.

Ce théorème a été démontré par I.Badulescu dans sa thèse (voir [Ba1] ou [Ba2]). Auparavant, on disposait d'un résultat plus faible, dû à G.Henniart, qui figure en appendice dans [He1] (Henniart définit dans loc. cit. une injection de l'ensemble des représentations cuspidales de $\mathrm{GL}_{d}(K)$ dans $\mathcal{A}_{D_{d}}$, vérifiant les propriétés caractérisant $\mathrm{JL}$ ).

Dans ce qui suit, on considérera non pas des représentations complexes mais à valeur dans $\overline{\mathbb{Q}}_{\ell}$. La notion de représentation « essentiellement de carré intégrable 》 est une notion " algébrique » (c'est évident pour les cuspidales; pour les autres, cela résulte du cas cuspidal, du théorème de classification de Zelevinski des séries discrètes pour $\mathrm{GL}_{d}$ et de considérations sur la rationalité du processus d'induction). On peut donc transporter la bijection du théorème précédent, via l'isomorphisme $\overline{\mathbb{Q}}_{\ell} \simeq \mathbb{C}$ choisi : la correspondance de Jacquet-Langlands locale est équivariante sous l'action de $\operatorname{Aut}(\mathbb{C})$ parce que les caractères des représentations le sont. Désormais, les éléments de $\mathcal{A}_{D_{d}}$ et $\mathcal{A}_{d}^{d}(K)$ seront vues comme des $\overline{\mathbb{Q}}_{\ell}$-représentations.

Pour finir, soulignons que la correspondance de Jacquet-Langlands locale, qui intervient dans la formulation de la conjecture de Drinfeld-Carayol, ne sera exploitée que dans le contexte global du lemme 10.3 .

9.1.2. La correspondance de Langlands locale. - Pour tout entier $d \geq 1$, désignons par $\mathcal{A}_{d}^{0}(K)$ l'ensemble des (classes d'équivalence de) représentations cuspidales irréductibles à caractère central d'ordre fini de $\mathrm{GL}_{d}(K)$, et par $\mathcal{G}_{d}^{0}(K)$ l'ensemble des (classes d'équivalences de) représentations continues complexes irréductibles de degré $d$ du groupe $W_{K}$, de caractère central d'ordre fini.

\section{Théorème 9.2 (Laumon, Rapoport et Stuhler, en égale caractéristique $p$ )}

Il existe une famille de bijections :

$$
\begin{aligned}
\mathcal{A}_{d}^{0}(K) & \longrightarrow \mathcal{G}_{d}^{0}(K) \\
\pi & \longmapsto \sigma_{d}(\pi)
\end{aligned}
$$

caractérisée par les propriétés suivantes :

(i) le déterminant de $\sigma_{d}(\pi)$ correspond par l'isomorphisme de la théorie du corps de classes local au caractère central de $\pi$. En particulier, $\sigma_{1}$ est donné par cet isomorphisme;

(ii) (torsion) pour tout quasi-caractère $\chi \in \mathcal{A}_{d}^{0}(K)$, on a :

$$
\sigma_{d}(\pi \otimes \chi)=\sigma_{d}(\pi) \otimes \sigma_{1}(\chi)
$$


(iii) pour chaque paire de représentations $\pi \in \mathcal{A}_{d}^{0}(K), \pi^{\prime} \in \mathcal{A}_{d^{\prime}}^{0}(K)$, on a l'identité des facteurs locaux :

$$
L\left(\sigma_{d}(\pi) \otimes \sigma_{d^{\prime}}\left(\pi^{\prime}\right), s\right)=L\left(\pi \times \pi^{\prime}, s\right), \quad \epsilon\left(\sigma_{d}(\pi) \otimes \sigma_{d^{\prime}}\left(\pi^{\prime}\right), s, \psi\right)=\epsilon\left(\pi \times \pi^{\prime}, s, \psi\right)
$$

(où $\psi$ désigne un caractère additif non trivial de $K$ );

(iv) (contragrédiente) pour $\pi \in \mathcal{A}_{d}^{0}(K)$, on a $: \sigma_{d}\left(\pi^{\vee}\right)=\sigma_{d}(\pi)^{\vee}$.

A vrai dire, le théorème précédent est démontré dans $[\mathbf{L}-\mathbf{R}-\mathbf{S}]$ non pas pour des représentations à valeurs complexes, mais pour des représentations $\ell$-adiques : la géométrie des variétés de modules des $\mathcal{D}$-faisceaux elliptiques fournit ( $\operatorname{sur} \overline{\mathbb{Q}}_{\ell}$ ) la correspondance dite « de Hecke » $r_{d}(\pi)=\sigma_{d}(\pi) \otimes||^{\frac{1-d}{2}}$ (c.f. paragraphe 10.1). En fait, cela revient au même : tant du côté automorphe que galoisien, les objets considérés admettent des définitions purement algébriques, et on peut transporter la bijection via l'isomorphisme $\overline{\mathbb{Q}}_{\ell} \simeq \mathbb{C}$ choisi (la correspondance de Hecke est invariante par Aut(C) : voir [He3 $)$. A partir de maintenant, on regardera $\mathcal{A}_{d}^{0}(K)$ et $\mathcal{G}_{d}^{0}(K)$ comme des ensembles de $\overline{\mathbb{Q}}_{\ell}$-représentations (une représentation complexe $\sigma \in \mathcal{G}_{d}^{0}(K)$, par continuité et par le fait que son caractère central est d'ordre fini, se factorise par un quotient fini, de sorte que la représentation sur $\overline{\mathbb{Q}}_{\ell}$ qui lui correspond est bien continue pour la topologie $\ell$-adique).

Soulignons également que la correspondance de Langlands locale, qui apparaît dans la formulation de la conjecture de Drinfeld-Carayol, sera utilisée dans ce texte uniquement sous la forme de la proposition 10.5, due à Laumon-Rapoport et Stuhler.

9.2. La représentation locale fondamentale. - Reprenant les notations et définition du paragraphe 7, on rappelle que $\Omega^{d}$ désigne le semi-plan supérieur généralisé de Drinfeld de dimension $d-1$ sur $K$. Il admet un système projectif $\left\{\Sigma_{n}^{d}\right\}_{n}$ de revêtements étales Galoisiens de $\Omega^{d} \otimes_{K} \widehat{K}^{n r}$. L'action semi-linéaire du groupe produit $\mathrm{GL}_{d}(K) \times D_{d}{ }^{*}$ sur $\Omega^{d} \otimes_{K} \widehat{K}^{n r}$ est obtenu en tordant l'action naturelle de $\mathrm{GL}_{d}(K)$ sur $\Omega^{d}$ (factorisée à travers $\mathrm{PGL}_{d}(K)$ ) par l'action suivante sur $\widehat{K}^{n r}$ :

$$
(g, b) \in \mathrm{GL}_{d}(K) \times D_{d}{ }^{*} \longmapsto \varphi_{q}^{\operatorname{val}(\operatorname{det}(g) \cdot N(b))},
$$

où l'on note $\varphi_{q} \in \operatorname{Gal}\left(K^{n r} / K\right)$ le Frobenius géométrique et $N: D_{d}{ }^{*} \rightarrow K^{*}$ la norme réduite. Cette action se relève en une action sur les revêtements $\Sigma_{n}^{d}$.

Considérons la restriction des scalaires, de $\widehat{K}^{n r}$ à $K$, de l'espace $\Sigma_{n}^{d}$ : si l'on étend à nouveau les scalaires à $\widehat{K}^{n r}$, on obtient un $\widehat{K}^{n r}$-espace rigide qui est l'union disjointe des $\Sigma_{n}^{d} \otimes_{\widehat{K}^{n r}, \sigma} \widehat{K}^{n r}$, où $\sigma$ varie au sein du groupe de Galois $\operatorname{Gal}\left(K^{n r} / K\right)$. Cet espace est trop gros, de sorte que l'on considère plutôt la restriction des scalaires « à la Weil », au sens suivant :

$$
\operatorname{Res}_{\widehat{K}^{n r} / K}^{\prime} \Sigma_{n}^{d}=\bigsqcup_{r \in \mathbb{Z}} \Sigma_{n}^{d} \otimes_{\widehat{K}^{n r}, \varphi_{q}^{r}} \widehat{K}^{n r} .
$$

C'est un espace rigide sur $\widehat{K}^{n r}$, muni d'une donnée de descente à $K$ définie sur le groupe de Weil uniquement. Désignons par $\left(\operatorname{Res}_{\widehat{K}^{n r} / K}^{\prime} \Sigma_{n}^{d}\right)_{\bar{K}}$ l'espace obtenu par extension des scalaires à $\widehat{\bar{K}}$ (c'est l'union disjointe de ses composantes $\left.\Sigma_{n}^{d} \otimes_{\widehat{K}^{n r}, \varphi_{q}^{r}} \widehat{\bar{K}}\right)$. L'action de $W_{K} \operatorname{sur}\left(\operatorname{Res}_{\widehat{K}^{n r} / K}^{\prime} \Sigma_{n}^{d}\right)_{\bar{K}}$ est la suivante : le groupe d'inertie $I_{K}$ agit de manière évidente sur chaque composante; de plus, $w \in W_{K}$ translate ces composantes indexées par $\mathbb{Z}$ par $+\operatorname{val}\left(\mathrm{Cl}(w)^{-1}\right)$, où l'on note $\mathrm{Cl}: W_{K} \rightarrow K^{*}$ l'application de réciprocité de la théorie du corps de classe (normalisée de telle manière qu'au Frobenius $\varphi_{q}$ corresponde l'uniformisante $\varpi$ ). Cette action commute avec la précédente, d'où une action du groupe produit $G=\mathrm{GL}_{d}(K) \times D_{d}{ }^{*} \times W_{K} \operatorname{sur}\left(\operatorname{Res}_{\widehat{K}^{n r} / K}^{\prime} \Sigma_{n}^{d}\right)_{\bar{K}}$. 
Le stabilisateur d'une composante $\left(\Sigma_{n}^{d}\right)_{\bar{K}}$ est le sous-groupe $\mathfrak{P}_{d}$ constitué des triplets $(g, b, w)$ qui vérifient $\operatorname{det}(g) N(b) \mathrm{Cl}(w)^{-1} \in \mathcal{O}^{*}$.

Considérons enfin la cohomologie $\ell$-adique à support compact (définie par Berkovich; voir paragraphe A.1) du $K$-espace analytique $\operatorname{Res}_{\widehat{K}^{n r} / K}^{\prime} \Sigma_{n}^{d}$ :

$$
\Psi_{d, n}^{i}=\mathrm{H}_{c}^{i}\left(\left(\operatorname{Res}_{\widehat{K}^{n r} / K}^{\prime} \Sigma_{n}^{d}\right)_{\bar{K}}, \overline{\mathbb{Q}}_{\ell}\right) .
$$

Définition 9.3. - Nous appelons représentation locale fondamentale la limite inductive $\Psi_{d}^{i}=$ $\underset{n}{\lim } \Psi_{d, n}^{i}$, pour $i=d-1$ (cohomologie en degré médian).

Il s'agit d'une représentation $\ell$-adique du groupe $G$; en fait, on voit facilement que $\Psi_{d}^{i}$ est l'induite compacte de $\mathfrak{P}_{d}$ à $G$ de la limite sur $n$ des représentations $\mathrm{H}_{c}^{i}\left(\left(\Sigma_{n}^{d}\right)_{\bar{K}}, \overline{\mathbb{Q}}_{\ell}\right)$. C'est de cette dernière manière qu'est définie - traditionnellement - la représentation locale fondamentale (cf. [Ca2]).

9.3. Enoncé de la conjecture. - Fixons un caractère d'ordre fini $\xi$ de $K^{*}$ à valeurs dans $\overline{\mathbb{Q}}_{\ell}^{*}$. On note $\Psi_{d}^{i}(\xi)$ la composante $\xi$-isotypique de $\Psi_{d}^{i}$, c'est-à-dire le plus grand quotient où le centre $K^{*}$ de $\mathrm{GL}_{d}(K)$ (ou de $D^{*}$ ) agit par $\xi$ : un élément $z \in K^{*}$ agit donc sur $\Psi_{d}^{i}(\xi)$ via le scalaire $\xi(z)$.

Notons $G$ le groupe produit $\mathrm{GL}_{d}(K) \times D_{d}{ }^{*} \times W_{K}$. On dira qu'une représentation $\Pi$ de $G$ (sur $\overline{\mathbb{Q}}_{\ell}$ ) est admissible si elle provient par extension des scalaires à $\overline{\mathbb{Q}}_{\ell}$ d'une représentation $\Pi_{E}$ de $G$ définie sur une extension finie $E$ de $\mathbb{Q}_{\ell}$, la représentation $\Pi_{E}$ étant une représentation admissible (au sens usuel) du groupe produit $H=\mathrm{GL}_{d}(K) \times D_{d}{ }^{*}$ telle que, pour tout compact $M \subset H$, l'action de $W_{K}$ sur l'espace $\Pi_{E}^{M}$ des invariants sous $M$ est continue. Nous notons $\mathcal{A}_{G}$ la catégorie des $\overline{\mathbb{Q}}_{\ell}$-représentations admissibles de $G$. Les objets irréductibles $\Pi$ de $\mathcal{A}_{G}$ sont les produits tensoriels $\pi \otimes \rho \otimes \sigma$, où $\pi$ (resp. $\rho$ ) est une représentation irréductible admissible de $\mathrm{GL}_{d}(K)$ (resp. $D^{*}$ ) définie sur une extension finie $E$ de $\mathbb{Q}_{\ell}$ (dépendant de $\Pi$ ) et $\sigma$ une représentation $\ell$-adique irréductible de $W_{K}$ définie sur $E$. Le lecteur pourra consulter, par exemple, la thèse de Boyer ( Boy $)$ pour une preuve détaillée de cette affirmation.

Proposition 9.4. - Les représentations $\Psi_{d}^{i}(\xi)$ appartiennent à la catégorie $\mathcal{A}_{G}$.

Ce fait sera démontré plus loin (cf. proposition 10.6 (i.)), à l'exception de l'admissibilité de l'action de $\mathrm{GL}_{d}(K)$ qui découle de Boy et [Fa1] (l'auteur ne sait pas démontrer directement que l'action de $\mathrm{GL}_{d}$ est admissible; noter cependant que cette admissibilité n'intervient pas dans la preuve du théorème qui suit). Il repose principalement sur des résultats de Berkovich. La conjecture de Drinfeld-Carayol précise alors la décomposition de $\Psi_{d}^{i}(\xi)$ :

Théorème 9.5. - Soit $\pi$ une représentation irréductible supercuspidale de $\mathrm{GL}_{d}(K)$, de caractère central $\xi$. Alors $\pi$ intervient dans la décomposition en éléments irréductibles de $\Psi_{d}^{i}(\xi)$ si et seulement si $i=d-1$. La composante $\pi$-isotypique de la représentation locale fondamentale est :

$$
\Psi_{d}^{d-1}(\xi)[\pi]=\mathrm{JL}(\pi) \otimes\left(\sigma_{d}(\pi) \otimes||^{\frac{1-d}{2}}\right) .
$$

On rappelle que, pour $W$ un objet de $\mathcal{A}_{G}$ et $\pi$ une représentation irréductible de $\mathrm{GL}_{d}(K)$, la composante $\pi$-isotypique de $W$ est définie par

$$
W[\pi]=\operatorname{Hom}_{\mathrm{GL}_{d}(K)}(\pi, W) .
$$


Remarque. - Qu'en est-il de la partie non supercuspidale de la cohomologie? La conjecture suivante est due à Carayol (cas $b=0$ ) et à Harris (en dehors du degré médian) :

Conjecture 9.6 (Carayol-Harris). -

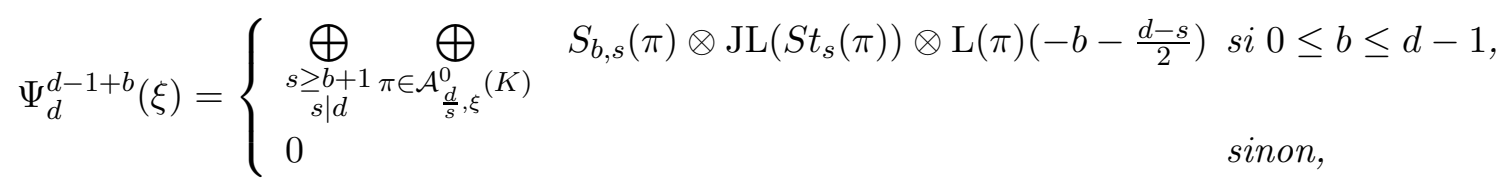

où $S t_{s}(\pi)$ désigne la représentation de Steinberg généralisée et où $S_{b, s}(\pi)$ est l'unique sous-module irréductible de l'induite normalisée $\pi||^{\frac{1-s}{2}} \times \cdots \times \pi||^{\frac{1-s}{2}+b-1} \times S t_{s-b}(\pi)||^{\frac{b}{2}}(0 \leq b \leq s-1)$ (toutes ces représentations sont des sous-quotients de $\pi||^{\frac{1-s}{2}} \times \cdots \times \pi||^{\frac{s-1}{2}}$ ).

\section{Preuve de la conjecture de Drinfeld-Carayol}

10.1. Cohomologie de la variété modulaire $\mathcal{E} \ell_{X, \mathcal{D}, I}$ et correspondance de Langlands locale. - Nous rappelons ici les résultats fondamentaux établis par Laumon, Rapoport et Stuhler dans [L-R-S]. Pour alléger les formules, nous noterons simplement $\mathcal{E} \ell_{I}$ les schémas $\mathcal{E} \ell \ell_{X, \mathcal{D}, I}$, et $\mathcal{E} \ell \ell_{I, F}=\mathcal{E} \ell \ell_{I} \times_{X} \operatorname{Spec} F$.

Fixons un nombre premier $\ell \neq p$; on s'intéresse aux espaces de cohomologie $\ell$-adique

$$
H_{I}^{n}=\mathrm{H}^{n}\left(\mathcal{E} \ell \ell_{I, F} \otimes_{F} \bar{F}, \overline{\mathbb{Q}}_{\ell}\right) \quad(0 \leq n \leq 2 d-2) .
$$

Ce sont des $\overline{\mathbb{Q}}_{\ell}$-espaces vectoriels de dimension finie, munis d'une $\mathbb{Q}_{\ell}$-structure et d'une action (continue) du groupe de Galois $\operatorname{Gal}\left(F^{s} / F\right)$. Par ailleurs, l'action à droite de $D^{*}\left(\mathbb{A}^{\infty}\right)$ sur $\mathcal{E} \ell \ell_{F}$ (définie via les opérateurs de Hecke, cf. 86.3 ) induit une action à gauche (définie sur $\mathbb{Q}_{\ell}$ et commutant à celle de Galois) sur la limite inductive $H^{n}=\underset{I \not \supset \infty}{\lim _{I}} H_{I}^{n}$. Le groupe produit $D^{*}\left(\mathbb{A}^{\infty}\right) \times$ $\operatorname{Gal}\left(F^{s} / F\right)$ opère donc sur $H^{n}$.

Dans ce qui suit, seul nous intéresse l'action du produit $D^{*}\left(\mathbb{A}^{\infty}\right) \times W_{F_{o}}$, où $o \neq \infty$ est une place fixée de $F$; on considérera plutôt les espaces de cohomologie

$$
H_{o, I}^{n}=\mathrm{H}^{n}\left(\mathcal{E} \ell \ell_{I, F} \otimes_{F} \overline{F_{o}}, \overline{\mathbb{Q}}_{\ell}\right),
$$

ainsi que leur limite inductive $H_{o}^{n}$. D'après le théorème de changement de base propre, $H_{o}^{n}$ est canoniquement isomorphe à $H^{n}$ en tant que $\overline{\mathbb{Q}}_{\ell}$-représentation de $W_{F_{o}}$. Comme $\left(H_{o}^{n}\right)^{K_{I}^{\infty}}=H_{o, I}^{n}$ est de dimension finie (avec les notations du paragraphe 6.3), l'action de $D^{*}\left(\mathbb{A}^{\infty}\right)$ sur $H_{o}^{n}$ est admissible.

Considérons les semi-simplifiés $\left(H_{o}^{n}\right)^{s s}$ des $H_{o}^{n}$. Ces représentations se décomposent en composantes isotypiques suivant les (classes d'équivalence de) représentations admissibles irréductibles $\Pi^{\infty}$ de $D^{*}\left(\mathbb{A}^{\infty}\right)$ :

$$
\left(H_{o}^{n}\right)^{s s}=\bigoplus_{\Pi^{\infty}} \Pi^{\infty} \otimes V_{\Pi^{\infty}}^{n}
$$

où les $V_{\Pi^{\infty}}^{n}$ sont des représentations $\ell$-adiques de dimension finie de $W_{F_{o}}$. Le résultat central de [L-R-S décrit ces décompositions (où l'on note St la représentation de Steinberg) :

Théorème 10.1. - (cf. [L-R-S] théorème 14.9 et 14.12) Seules peuvent intervenir non trivialement, dans les décompositions isotypiques des $\left(H_{o}^{n}\right)^{\text {ss }}$, des représentations telles que, ou bien $\Pi^{\infty} \otimes 1$, ou bien $\Pi^{\infty} \otimes \mathrm{St}_{\infty}$, soit automorphe. Dans le premier cas, $\Pi^{\infty}$ se factorise sous la forme $\chi^{\infty} \circ \mathrm{Nr}$, où $\chi^{\infty}$ est un caractère de $\left(\mathbb{A}^{\infty}\right)^{*}$ tel que $\chi^{\infty} \otimes 1$ soit un caractère de Hecke de $\mathbb{A}^{*}$. Dans le second cas, les $V_{\Pi^{\infty}}^{n}$ son nuls pour $n \neq d-1$, tandis que $V_{\Pi^{\infty}}^{d-1}$ est une représentation 
semi-simple de dimension $m(\Pi) d$ de $W_{F_{o}}(m(\Pi)$ désigne la multiplicité de $\Pi$; voir loc. cit. pour les propriétés caractérisant $\left.V_{\Pi \infty}^{d-1}\right)$.

Expliquons comment Laumon, Rapoport et Stuhler construisent l'application $\pi \mapsto \sigma_{d}(\pi)$ de la correspondance de Langlands locale (cf. paragraphe 9.1.2), en faisant usage du théorème précédent. Partant du corps local $K$, on choisit un corps global $F$ et une place $o$ de $F$ tel que le complété $F_{o}$ soit isomorphe à $K$ (nous fixons un tel isomorphisme). Choisissons ensuite deux autres places $\infty$ et $o^{\prime}$ (distinctes et distinctes de $o$ ). Fixant l'entier $d \geq 1$, nous notons $D$ l'algèbre à division de centre $F$, de dimension $d^{2}$ et d'invariants :

$$
\operatorname{inv}_{x}(D)= \begin{cases}1 / d & \text { si } x=o \\ -1 / d & \text { si } x=o^{\prime} \\ 0 & \text { sinon. }\end{cases}
$$

Le lemme suivant, démontré dans $[\mathbf{L}-\mathbf{R}-\mathbf{S}]$ au moyen d'une formule des traces de Selberg simplifiée, permet de voir toute $\pi \in \mathcal{A}_{n}^{0}(K)$ comme composante en $o$ d'une représentation automorphe de $\mathrm{GL}_{d}(\mathbb{A})$ :

Lemme 10.2. - (cf. [L-R-S 15.10) Donnons-nous $\pi \in \mathcal{A}_{n}^{0}(K)$ et une place $x \notin\left\{o, o^{\prime}, \infty\right\}$ de F. Il existe alors une représentation automorphe parabolique $\widetilde{\Pi}=\otimes \widetilde{\Pi}_{v}$ de $\mathrm{GL}_{d}(\mathbb{A})$ telle que $\widetilde{\Pi}_{\infty} \simeq \mathrm{St}_{\infty}, \widetilde{\Pi}_{o} \simeq \pi$, et que $\widetilde{\Pi}_{o^{\prime}}$ et $\Pi_{x}$ soient cuspidales.

Puis on transfert $\widetilde{\Pi}$ au groupe $D^{*}\left(\mathbb{A}^{\infty}\right)$; c'est un cas particulier de la correspondance de Jacquet-Langlands globale, qu'on aimerait bien faire marcher en général :

Lemme 10.3. - (cf. [L-R-S] 15.11) Soit $\widetilde{\Pi}$ comme dans le lemme précédent. Il existe alors une unique représentation automorphe $\Pi$ de $D^{*}(\mathbb{A})$ telle que, pour tout $v \notin\left\{o, o^{\prime}\right\}$, on ait $\Pi_{v} \simeq \widetilde{\Pi}_{v}$. On a $\Pi_{v} \simeq \mathrm{JL}\left(\widetilde{\Pi}_{v}\right)$, pour $v=$ o et $o^{\prime}$. De plus, la multiplicité $m(\Pi)$ vaut 1 .

Remarque. - Ces deux lemmes sont (respectivement) des cas particuliers des deux assertions du théorème suivant; la démonstration m'en a été indiquée par G. Henniart et I. Badulescu:

Théorème 10.4. - Soit $S$ un ensemble fini de places de $F$.

1. (cf. [Ba1] théorème 1.11.8) Supposons que pour tout $v \in S$ on se donne une représentation essentiellement de carré intégrable $\pi_{v}$ de $\mathrm{GL}_{d}\left(F_{v}\right)$. Alors il existe une représentation automorphe cuspidale $\widetilde{\Pi}$ de $\mathrm{GL}_{d}(\mathbb{A})$ telle que, pour tout $v \in S$, on ait $\widetilde{\Pi}_{v} \simeq \pi_{v}$.

2. (cf. [He1 Appendix A.4) Supposons que l'algèbre à division D soit scindée en dehors de $S$. Donnons-nous une représentation automorphe cuspidale $\widetilde{\Pi}$ de $\mathrm{GL}_{d}(\mathbb{A})$ telle que $\widetilde{\Pi}_{v}$ soit essentiellement de carré intégrable en toute place $v \in S$ et qu'en une place $v \notin S$ au moins $\widetilde{\Pi}_{v}$ soit cuspidale. Alors il existe une unique représentation automorphe $\Pi$ de $D^{*}(\mathbb{A})$ telle que $\Pi^{S} \simeq \widetilde{\Pi}^{S}$. Elle vérifie $: \Pi_{v} \simeq \widetilde{\Pi}_{v}$ si $D$ est déployée en $v$ et $\Pi_{v} \simeq \mathrm{JL}\left(\widetilde{\Pi}_{v}\right)$ si $D_{v}$ est ramifiée. De plus, la multiplicité $m(\Pi)$ vaut 1.

Le point 2. se démontre en appliquant la formule des traces simples de Deligne-Kazhdan (voir Boy preuve de la prop. 15.4).

On applique maintenant le théorème 10.1 : il correspond à $\Pi$ une représentation $V_{\Pi \infty}^{d}$ de dimension $d$ du groupe de Weil $W_{F_{o}}$. Notons $\Sigma(\Pi)$ la représentation obtenue en tordant $V_{\Pi^{\infty}}^{d}$ par ||$^{\frac{d-1}{2}}$ (cette opération rend le déterminant d'ordre fini), et $\Sigma(\pi)$ l'ensemble des représentations $\Sigma(\Pi)$, pour tous les choix possibles de $\widetilde{\Pi}$. Laumon, Rapoport et Stuhler démontrent que, pour tout $\pi \in \mathcal{A}_{d}^{0}(K)$, l'ensemble $\Sigma(\pi)$ est réduit à un seul élément $\sigma(\pi)$, que l'application $\pi \mapsto \sigma(\pi)$ 
est injective et qu'elle satisfait à toutes les propriétés requises. Ils concluent par la conjecture de Langlands locale numérique, prouvée par Henniart.

Pour notre application, nous retiendrons :

Proposition 10.5. - Soit $\Pi$ la représentation automorphe de $D^{*}(\mathbb{A})$ obtenue à partir de $\pi$ en appliquant les lemmes 10.2 et 10.3. Alors la composante $\Pi^{\infty, o}$-isotypique de $\left(H_{o}^{n}\right)^{\text {ss }}$ est nulle sauf pour $n=d-1$. Dans ce dernier cas, on $a$ :

$$
\left(\left(H_{o}^{d-1}\right)^{s s}\left[\Pi^{\infty, o}\right]\right)_{o}=\mathrm{JL}(\pi) \otimes\left(\sigma_{d}(\pi) \otimes||^{\frac{1-d}{2}}\right) .
$$

10.2. Construction de la suite spectrale de Hochschild-Serre. - A partir de maintenant, nous fixons une place $o$ de $F$ telle que le complété $D_{o}=D \otimes F_{o}$ en $o$ soit « le » corps gauche sur $F_{o}$ d'invariant $1 / d$ (noté précédemment $D_{d} ; F_{o}$ jouera par la suite le rôle du corps local $K$ du paragraphe 9.3). Par ailleurs, fixons un nombre premier $\ell$ distinct de la caractéristique $p$ du corps résiduel de $F_{o}$, ainsi qu'une clôture algébrique $\bar{F}_{o}$ de $F_{o}$. L'objet de cette section est de démontrer la :

Proposition 10.6. - (i) Pour tout entier $0 \leq j \leq 2(d-1)$, le groupe de cohomologie à support compact $\mathrm{H}_{c}^{j}\left(\left(\operatorname{Res}^{\prime} \Sigma_{n}^{d}\right)_{\bar{F}_{o}}, \mathbb{Q}_{\ell}\right)$ est une $\mathbb{Q}_{\ell}$-représentation lisse du groupe produit $\mathrm{GL}_{d}\left(F_{o}\right) \times D_{o}^{*}$. Elle est de type fini en tant que $\mathrm{GL}_{d}\left(F_{o}\right)$-module.

(ii) La cohomologie $\ell$-adique de l'espace analytique $\operatorname{Res}^{\prime} \Sigma_{n}^{d}$ et la cohomologie $\ell$-adique du schéma $\mathcal{E} \ell_{X, \mathcal{D}, I}$ sont reliées par la suite spectrale :

$$
\begin{gathered}
\mathrm{E}_{2}^{i, j}=\operatorname{Ext}_{\mathrm{GL}_{d}\left(F_{o}\right)-\text { mod. lisse }}^{i}\left(\mathrm{H}_{c}^{2(d-1)-j}\left(\left(\operatorname{Res}^{\prime} \Sigma_{n}^{d}\right)_{\bar{F}_{o}}, \mathbb{Q}_{\ell}\right)(d-1), \mathcal{A}_{I^{o}}^{\infty}\right) \\
\Longrightarrow \mathrm{H}^{i+j}\left(\mathcal{E} \ell \ell_{X, \mathcal{D}, I} \otimes \bar{F}_{o}, \mathbb{Q}_{\ell}\right),
\end{gathered}
$$

où $\mathcal{A}_{I^{\circ}}^{\infty}$ est l'espace des formes automorphes sur $Z_{I^{\circ}}$ triviales à l'infini et où $(d-1)$ indique comme toujours un twist de Tate. De plus, les suites spectrales obtenues lorsque I varie (donc également la multiplicité $n$ de o dans I) sont compatibles avec les applications de transition; les systèmes projectifs obtenus sont équivariants pour l'action de $D^{*}\left(\mathbb{A}^{\infty}\right)$ et celle du groupe de Weil $W_{F_{o}}$.

En passant à la limite projective sur $I^{o}$, on obtient le corollaire suivant :

Corollaire 10.7. - Il existe une suite spectrale $D^{*}\left(\mathbb{A}^{\infty}\right) \times W_{F_{o}}$-équivariante :

$$
\begin{gathered}
\mathrm{E}_{2}^{i, j}=\operatorname{Ext}_{\mathrm{GL}_{d}\left(F_{o}\right)-\text { mod. lisse }}^{i}\left(\mathrm{H}_{c}^{2(d-1)-j}\left(\left(\operatorname{Res}^{\prime} \Sigma_{n}^{d}\right)_{\bar{F}_{o}}, \mathbb{Q}_{\ell}\right)(d-1), \mathcal{A} \bar{D}\right) \\
\Longrightarrow \mathrm{H}^{i+j}\left(\mathcal{E} \ell \ell_{n}^{\infty} \otimes \bar{F}_{o}, \mathbb{Q}_{\ell}\right),
\end{gathered}
$$

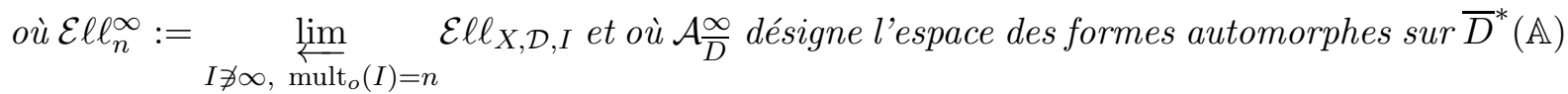
triviales à l'infini. Les suites spectrales obtenues lorsque $n$ varie sont compatibles avec les applications de transition et avec les actions.

Remarque 10.8. - Attention, dans les suites spectrales de la proposition 10.6 et son corollaire, l'action de $\mathrm{GL}_{d}\left(F_{o}\right)$ sur la cohomologie à support des revêtements $\Sigma_{n}^{d}$ est celle de Drinfeld composée avec l'application $g \mapsto{ }^{t} g^{-1}$; cela provient de l'application du théorème d'uniformisation 8.3 (voir paragraphe 10.2.3). 
Remarque. - Depuis ce travail, Fargues a écrit une suite spectrale valable dans tous les cas d'uniformisation connus (dont le nôtre) : voir $\mathbf{F a r}$ thm. 4.2.1. Son approche, différente, requiert la théorie de Huber; de notre côté, nous utilisons celle, due à Berkovich, des espaces analytiques avec opérateurs.

Nous procédons de manière similaire à [H1 $\S 2$.

10.2.1. Définition d'un recouvrement admissible de $\operatorname{Res}^{\prime} \Sigma_{n}^{d}$ par des ouverts distingués. - Soit $\Delta$ l'immeuble de Bruhat-Tits de $\mathrm{PGL}_{d}\left(F_{o}\right)$ et $\Delta_{\mathbb{R}}$ sa réalisation géométrique (qui s'identifie aux classes de proportionnalité de normes sur le $F_{o}$-espace vectoriel $F_{o}^{d}$ ). Il existe une application $\mathrm{GL}_{d}\left(F_{o}\right)$-équivariante $\lambda: \Omega^{d} \rightarrow \Delta_{\mathbb{R}}$, dont l'image est $\Delta_{\mathbb{Q}}$ et qui permet de définir la structure rigide-analytique sur le demi-plan généralisé de Drinfeld $\Omega^{d}$ (voir [Dr4], [Bo-Ca ou [De-Hu] $)$. Nous utilisons cette application afin de définir un recouvrement admissible de $\Omega^{d}$ ainsi que des revêtements $\Sigma_{n}^{d}$. Fixons $0<\varepsilon<\frac{1}{2}$; pour tout sommet $s \in \Delta$, on considère le $\varepsilon$-voisinage $V(s, \varepsilon)$ de l'étoile de $s$ dans la première subdivision barycentrique. Les ensembles $U_{0}(s, \varepsilon):=$ $\lambda^{-1}(V(s, \varepsilon))$ constituent un recouvrement de $\Omega^{d}$ par des ouverts distingués, au sens de Berkovich (ils sont de la forme $V \backslash U$, avec $U \subset V$ des polydisques, cf. loc. cit.). Parce que les morphismes $\lambda_{n}$ : $\Sigma_{n}^{d} \rightarrow \Omega^{d} \hat{\otimes} \widehat{F}_{o}^{n r}$ sont étales, les revêtements $\Sigma_{n}^{d}$ sont donc recouverts par les ouverts analytiques distingués $U_{n}(s)=\lambda_{n}^{-1}\left(U_{0}(s, \varepsilon)\right)$, lorsque $s$ décrit l'ensemble des sommets de l'immeuble $\Delta$.

Notons $\Delta_{i}$ l'ensemble des simplexes de $\Delta$ de dimension $i$. Pour $\delta=\left(s_{1}, \ldots, s_{i}\right)$ dans $\Delta_{i}$, on définit $V(\delta, \varepsilon)=\bigcap_{k=1}^{i} V\left(s_{k}, \varepsilon\right) \subset \Delta_{\mathbb{R}}, U_{0}(\delta)=\lambda^{-1}(V(\delta, \varepsilon))$ et $U_{n}(\delta)=\lambda_{n}^{-1}\left(U_{0}(\delta)\right)=$ $\bigcap_{k=1}^{i} U_{n}\left(s_{k}\right)$. Finalement, soit $\varphi \in \operatorname{Gal}\left(F_{o}^{n r} / F_{o}\right)$ le Frobenius géométrique; pour tout entier $r \in \mathbb{Z}$, on considère l'ouvert distingué $U_{n}(\delta, r)=U_{n}(\delta) \otimes_{\widehat{F}_{o}^{n r}, \varphi^{r}} \widehat{F}_{o}^{n r}$ de $\Sigma_{n}^{d} \otimes_{\widehat{F}_{o}^{n r}, \varphi^{r}} \widehat{F}_{o}^{n r}$. Alors l'espace rigide-analytique $\operatorname{Res}^{\prime} \Sigma_{n}^{d}=\bigsqcup_{r \in \mathbb{Z}} \Sigma_{n}^{d} \otimes_{\widehat{F}_{o r}^{n r}, \varphi^{r}} \widehat{F}_{o}^{n r}$ est recouvert par $\left\{U_{n}(\delta, r)\right\}_{(\delta, r) \in \Delta_{0} \times \mathbb{Z}}$. C'est un recouvrement admissible localement fini, de nerf $\Delta \times \mathbb{Z}=\bigcup_{i}\left(\Delta_{i} \times \mathbb{Z}\right)$.

D'après le lemme A.3 de Berkovich, on a :

Fait 1. - Il existe une suite spectrale

$$
\mathrm{E}_{1}^{i, j}=\bigoplus_{\delta \in \Delta_{-i}} \bigoplus_{r \in \mathbb{Z}} \widetilde{\mathrm{H}}_{c}^{j}\left(U_{n}(\delta, r)_{\bar{F}_{o}}, \mathbb{Q}_{\ell}\right) \Longrightarrow \mathrm{H}_{c}^{i+j}\left(\left(\operatorname{Res}^{\prime} \Sigma_{n}^{d}\right)_{\bar{F}_{o}}, \mathbb{Q}_{\ell}\right)
$$

(rappel : nous notons $X_{\bar{F}_{o}}=X \hat{\otimes}_{\widehat{F}_{o} r} \widehat{\bar{F}}_{o}$, pour tout $\widehat{F}_{o}^{n r}$-espace analytique $X$ ).

10.2.2. calcul de $\mathrm{H}_{c}\left(\left(\operatorname{Res}^{\prime} \Sigma_{n}^{d}\right)_{\bar{F}_{o}}, \mathbb{Q}_{\ell}\right)$ à l'aide d'un complexe $\mathcal{P}$ de $\mathbb{Q}_{\ell}-\mathrm{GL}_{d}\left(F_{o}\right)$-modules projectifs lisses. - Le groupe $\mathrm{GL}_{d}\left(F_{o}\right)$ permute les éléments du recouvrement $\left\{U_{n}(\delta, r)\right\}_{(\delta, r) \in \Delta_{0} \times \mathbb{Z}}$ : rappelons que l'action sur $\mathbb{Z}$ est donnée par $(g, r) \mapsto r+$ val det $g$. Pour $\delta$ un simplexe dans $\Delta$, on note $S(\delta) \subset \mathrm{GL}_{d}\left(F_{o}\right)$ le sous-groupe ouvert compact (modulo le centre) fixant $\delta$. Le stabilisateur de l'ouvert $U_{n}(\delta, r)$, ou encore celui de $(\delta, r)$, est alors $S^{\prime}(\delta)=$ $S(\delta) \cap \mathrm{GL}_{d}^{\prime}\left(F_{o}\right)$, où $\mathrm{GL}_{d}^{\prime}\left(F_{o}\right)$ désigne le sous-groupe de $\mathrm{GL}_{d}\left(F_{o}\right)$ constitué des éléments dont le déterminant est une unité de l'anneau des entiers $\mathcal{O}_{o}$. Ce stabilisateur $S^{\prime}(\delta)$ est un sous-groupe compact ouvert de $\mathrm{GL}_{d}\left(F_{o}\right)$.

Il est bien connu que le groupe $\mathrm{GL}_{d}\left(F_{o}\right)$ contient un pro-p-sous-groupe ouvert, où $p$ désigne la caractéristique résiduelle de $F_{o}$, qui est distincte du nombre premier $\ell$ que l'on s'est donné. On peut donc appliquer la proposition A.12 de l'appendice à l'ouvert distingué $U_{n}(\delta, r)$ du $\mathrm{GL}_{d}\left(F_{o}\right)$ espace $\operatorname{Res}^{\prime} \Sigma_{n}^{d}$ : l'action de $S^{\prime}(\delta)$ sur les groupes $\widetilde{\mathrm{H}}_{c}^{j}\left(U_{n}(\delta, r), \mathbb{Q}_{\ell}\right)$ est lisse; on sait construire un complexe $C_{n}^{\cdot}(\delta, r)$ de $\mathbb{Q}_{\ell}-S^{\prime}(\delta)$-modules lisses calculant la cohomologie :

$$
h^{j}\left(C_{n}^{\cdot}(\delta, r)\right) \stackrel{\sim}{\rightarrow} \widetilde{\mathrm{H}}_{c}^{j}\left(U_{n}(\delta, r)_{\bar{F}_{o}}, \mathbb{Q}_{\ell}\right) \quad \forall j \geq 0
$$


(isomorphismes $S^{\prime}(\delta)$-équivariants). La construction de ces complexes est fonctorielle : pour $\delta \subset \delta^{\prime}$ deux simplexes emboîtés et $r \in \mathbb{Z}$, on a des applications canoniques $C_{n}^{\cdot}\left(\delta^{\prime}, r\right) \rightarrow C_{n}^{\cdot}(\delta, r)$.

Posons $U_{n}(i)=\bigsqcup_{(\delta, r) \in \Delta_{-i} \times \mathbb{Z}} U_{n}(\delta, r)$ et $Z^{i, j}=\bigoplus_{(\delta, r) \in \Delta_{-i} \times \mathbb{Z}} C_{n}^{j}(\delta, r)$ (notons que les complexes $C_{n}(\delta, r)$ calculant la cohomologie avec support sont covariants pour l'inclusion, d'où le signe - dans la définition de $\left.Z^{i, j}\right)$, de sorte que

$$
h^{j}\left(Z^{-i, \cdot}\right) \stackrel{\sim}{\rightarrow} \widetilde{\mathrm{H}}_{c}^{j}\left(U_{n}(i)_{\bar{F}_{o}}, \mathbb{Q}_{\ell}\right) \quad \forall j \geq 0
$$

(isomorphismes $\mathrm{GL}_{d}\left(F_{o}\right)$-équivariants). Alors le complexe total $\mathcal{P} \cdot=\operatorname{Tot}\left(Z^{\cdot, \cdot}\right.$ ) associé au double complexe $Z$,' calcule la cohomologie de l'espace entier $\operatorname{Res}^{\prime} \Sigma_{n}^{d}$ :

Fait 2. - On a des isomorphismes $\mathrm{GL}_{d}\left(F_{o}\right)$-équivariants :

$$
h^{j}\left(\mathcal{P}^{\cdot}\right) \stackrel{\sim}{\rightarrow} \mathrm{H}_{c}^{j}\left(\left(\operatorname{Res}^{\prime} \Sigma_{n}^{d}\right)_{\overline{F_{o}}}, \mathbb{Q}_{\ell}\right) \quad \forall j \geq 0 .
$$

En effet, la situation est la suivante : les groupes $\widetilde{\mathrm{H}}_{c}^{j}\left(U_{n}(\delta, r)_{\bar{F}_{o}}, \mathbb{Q}_{\ell}\right)$ forment un système de coefficients (au sens de [G-M] Chap. I $\S 2.4$ ) contravariant sur le complexe simplicial $\Delta \times \mathbb{Z}$; le complexe résultant est le complexe de Čech $\check{C}_{c}\left(\mathcal{U}, \mathbb{Q}_{\ell}\right)$, où $\mathcal{U}$ désigne le recouvrement de Čech $\left\{U_{n}(\delta, r)\right\}$. Reprenons les notations du lemme A.14 de l'appendice : utilisant les résolutions

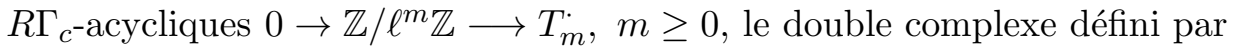

$$
\check{C}_{c}^{i}\left(\mathcal{U}, T^{j}\right):={\underset{m}{m}}_{\lim _{\mathrm{H}}}^{0}\left(U_{n}(i)_{\bar{F}_{o}}, T_{m}^{j}\right) \otimes_{\mathbb{Z}_{\ell}} \mathbb{Q}_{\ell}
$$

vérifie l'égalité suivante dans la catégorie dérivée des $\mathbb{Q}_{\ell}-\mathrm{GL}_{d}\left(F_{o}\right)$-modules :

$$
\operatorname{Tot}\left(\check{C}_{c}\left(\mathcal{U}, T^{\cdot}\right)\right)=R \Gamma_{c}\left(\left(\operatorname{Res}^{\prime} \Sigma_{n}^{d}\right)_{\overline{F_{o}}}, \mathbb{Q}_{\ell}\right)
$$

(c'est un résultat standard : voir [Go $\S 5.3$; l'amélioration essentielle par rapport au fait 1 est l'équivariance pour l'action de $\left.\mathrm{GL}_{d}\left(F_{o}\right)\right)$. Le fait 2 découle alors de la remarque A.16: il existe des quasi-isomorphismes équivariants canoniques (lorsque $i$ varie)

$$
Z^{-i \cdot \cdot} \longrightarrow \check{C}_{c}^{i}\left(\mathcal{U}, T^{\cdot}\right)
$$

Poursuivons : soit $O_{i}$ l'ensemble des orbites de $\Delta_{i} \times \mathbb{Z}$ sous l'action de $\mathrm{GL}_{d}\left(F_{o}\right)$. C'est un ensemble fini : on a $\left(\Delta_{i} \times \mathbb{Z}\right) / \mathrm{GL}_{d}\left(F_{o}\right) \simeq \bigsqcup_{\text {finie }} \mathbb{Z} /$ val $\operatorname{det}\left(S\left(\delta_{k}\right)\right)$, où l'on a choisi des représentants $\delta_{k}$ des différentes classes de $\Delta_{i} / \mathrm{GL}_{d}\left(F_{o}\right)$; comme $S\left(\delta_{k}\right)$ contient le centre de $\mathrm{GL}_{d}\left(F_{o}\right)$, dont l'image par val det est $d \mathbb{Z}$, on voit que le quotient est fini. Formellement, on a :

$$
Z^{i, j}=\bigoplus_{[\delta, r] \in O_{-i}} \mathrm{c}-\operatorname{Ind}_{S^{\prime}(\delta)}^{\mathrm{GL}_{d}\left(F_{o}\right)} C_{n}^{j}(\delta, r),
$$

où l'on note c-Ind l'induite compacte (cf. [B-Z] $)$. Cela résulte du lemme suivant :

Lemme 10.9. - Soit $V$ un k-espace vectoriel muni d'une action d'un groupe $G$ (localement compact, totalement discontinu) et $H$ un sous-groupe ouvert de $G$. On suppose que $V$ se décompose sous la forme

$$
V=\bigoplus_{\bar{g} \in G / H} V_{\bar{g}}
$$

de telle sorte que $g^{\prime}\left(V_{\bar{g}}\right) \subset V_{\overline{g^{\prime} g}} \forall\left(g^{\prime}, g\right) \in G^{2}$ et que $V_{\bar{g}}$ soit une représentation lisse de $H$. Alors $V$ s'identifie à c- $\operatorname{Ind}_{H}^{G} V_{\overline{1}}$ comme représentation de $G$. 
Démonstration. - A un élément $\left(v_{\bar{g}}\right)_{\bar{g} \in G / H}$ on associe la fonction

$$
\phi: g \in G \longmapsto g \cdot v \overline{g^{-1}}
$$

Cela définit une un morphisme $G$-équivariant entre $V$ et l'espace des fonctions $\phi: G \rightarrow V_{\overline{1}}$ qui vérifient :

i) $\phi(h g)=h \cdot \phi(g) \quad \forall(g, h) \in G \times H$;

ii) $\phi$ est à support dans un nombre fini de classes à gauche $H \backslash G$;

iii) $\phi$ est invariante à droite par l'action d'un sous-groupe ouvert de $G$.

C'est exactement de cette façon qu'est définie l'induite compacte.

Comme $C_{n}^{j}(\delta, r)$ est une représentation lisse du groupe compact $S^{\prime}(\delta)$, c'est une représentation finie (au sens de [B-Z 2.40), donc projective dans la catégorie des représentations lisses de $S^{\prime}(\delta)$ (voir lemme 10.15 plus loin). Puisque l'induite compacte conserve la lissité et la projectivité (cf. loc. cit. 2.29 : cela résulte de la réciprocité de Frobenius relativement à l'induite compacte), on voit que $\mathcal{P}$ ' est un complexe de $\mathrm{GL}_{d}\left(F_{o}\right)$-modules projectifs lisses.

Achevons la démonstration de la première assertion de la proposition 10.6]: la lissité de l'action de $D_{o}^{*}$ est évidente; il reste donc à montrer que $\mathrm{H}_{c}^{\bullet}\left(\Sigma_{n}^{d}\right):=\mathrm{H}_{c}^{\bullet}\left(\left(\operatorname{Res}^{\prime} \Sigma_{n}^{d}\right)_{\bar{F}_{o}}, \mathbb{Q}_{l}\right)$ est de type fini en tant que $\mathrm{GL}_{d}\left(F_{o}\right)$-module. On part de la suite spectrale figurant dans l'énoncé du fait 1, ou plutôt de sa version équivariante :

$$
\mathrm{E}_{1}^{i, j}=\bigoplus_{(\delta, r) \in \Delta_{-i} \times \mathbb{Z}} \widetilde{\mathrm{H}}_{c}^{j}\left(U_{n}(\delta, r)_{\bar{F}_{o}}, \mathbb{Q}_{l}\right) \Longrightarrow \mathrm{H}_{c}^{i+j}\left(\Sigma_{n}^{d}\right) .
$$

Elle se réécrit (vu précédemment) :

$$
\mathrm{E}_{1}^{i, j}=\bigoplus_{[\delta, r] \in O_{-i}} \mathrm{c}-\operatorname{Ind}_{S^{\prime}(\delta)}^{\mathrm{GL}_{d}\left(F_{o}\right)} \widetilde{\mathrm{H}}_{c}^{j}\left(U_{n}(\delta, r)_{\bar{F}_{o}}, \mathbb{Q}_{l}\right) \Longrightarrow \mathrm{H}_{c}^{i+j}\left(\Sigma_{n}^{d}\right) .
$$

Sachant que les $U_{n}(\delta, r)$ sont des ouverts distingués, les espaces vectoriels $\widetilde{\mathrm{H}}_{c}^{j}\left(U_{n}(\delta, r)_{\bar{F}_{o}}, \mathbb{Q}_{l}\right)$ sont de dimension finie (cf lemme A.1, dû à Berkovich). Ainsi $\mathrm{E}_{1}^{i, j}$ est une somme finie d'induites compactes de représentations de dimension finie; c'est donc une représentation de type fini de $\mathrm{GL}_{d}\left(F_{o}\right)$. D'après $\mathbf{B}-\mathbf{Z}$ 4.19, tout $\mathrm{GL}_{d}\left(F_{o}\right)$-module lisse est noethérien; par conséquent, $\mathrm{H}_{c}^{i+j}\left(\Sigma_{n}^{d}\right)$ possède une filtration finie $F^{i} \mathrm{H}_{c}^{i+j}\left(\Sigma_{n}^{d}\right)$ à quotients de type fini et est donc lui-même de type fini.

10.2.3. application du théorème d'uniformisation. - Rappelons que l'on note $Z_{I^{o}}$ l'ensemble des doubles classes

$$
Z_{I^{o}}=\bar{D}^{*}(F) \backslash \bar{D}^{*}\left(\mathbb{A}^{\infty}\right) / K_{I}^{\infty, o},
$$

où $K_{I}^{\infty, o}$ est un sous-groupe de congruences (voir paragraphe 8.1). Le groupe $\mathrm{GL}_{d}\left(F_{o}\right)$ agit $\operatorname{sur}\left(\operatorname{Res}^{\prime} \Sigma_{n}^{d}\right) \times Z_{I^{\circ}}$ et d'après le théorème d'uniformisation rigide-analytique 8.1 (ou plutôt la remarque 8.2; noter que l'on change le sens de l'action sur les revêtements, par composition de l'action de Drinfeld avec la transposition), on a un isomorphisme

$$
\mathcal{E} \ell \ell_{I}^{a n} \simeq\left[\left(\operatorname{Res}^{\prime} \Sigma_{n}^{d}\right) \times Z_{I^{o}}\right] / \mathrm{GL}_{d}\left(F_{o}\right)
$$

(la différence entre Res et $\operatorname{Res}^{\prime}$ est tuée dans le quotient). Notons $[\delta, r, z]$ la classe de $(\delta, r, z) \in$ $\Delta \times \mathbb{Z} \times Z_{I^{o}}$ sous $\mathrm{GL}_{d}\left(F_{o}\right)$ et $U_{n}[\delta, r, z] \subset \mathcal{E} \ell \ell_{I}^{a n}$ l'image de $U_{n}(\delta, r) \times\{z\}$ par l'application

$$
\left(\operatorname{Res}^{\prime} \Sigma_{n}^{d}\right) \times Z_{I^{o}} \stackrel{p}{\longrightarrow} \mathcal{E} \ell \ell_{I}^{a n}
$$

induite par l'isomorphisme (5). 
Fait 3. - Le groupe $\mathrm{GL}_{d}\left(F_{o}\right)$ agit librement sur $\Delta_{i} \times \mathbb{Z} \times Z_{I^{o}}$. De plus, pour $g \in \mathrm{GL}_{d}\left(F_{o}\right)$,

$$
\left(U_{n}(\delta g, r g) \times\{z g\}\right) \cap\left(U_{n}(\delta, r) \times\{z\}\right) \neq \emptyset \Longrightarrow g=1 .
$$

Pour démontrer ce fait, nous avons besoin du lemme suivant :

Lemme 10.10. - Soit $D$ une algèbre à division sur $F=\mathbb{F}_{q}(X)$ et $f \in \mathcal{O}_{D}^{*}$ vérifiant une relation de congruence non triviale. Alors $f$ n'est pas de torsion.

Démonstration. - Supposons $f^{n}=1$ et montrons que $f=1$. Écrivant $n=p^{\alpha} \beta(\beta \neq 0)$, il vient $f^{n}=\left(f^{\beta}\right)^{p^{\alpha}}$. On peut donc supposer que $n$ est premier à $p$ (injectivité du Frobenius dans $F(f))$. La relation de congruence, en une place $v$ où $D_{v}=\mathrm{GL}_{d}\left(\mathbb{F}_{q^{\prime}}((t))\right)$ pour un certain $d$ et un certain $q^{\prime}=q^{e}$, s'écrit $f=1+t^{r} M$, avec $r \geq 1$ et $M \in \mathrm{M}_{d}\left(\mathbb{F}_{q^{\prime}}[[t]]\right)$. Alors :

$$
f^{\beta}=1+\beta t^{r} M+(\text { termes de degré en } t \text { supérieur à } r \text { strictement }) \text {, }
$$

par la formule du binôme sur le corps commutatif $F(f)$. Ainsi $f^{\beta}=1$ si et seulement si $M=0$, donc si et seulement si $f=1$.

Démontrons maintenant le fait 3 : puisque l'action de $\mathrm{GL}_{d}\left(F_{o}\right)$ sur $\mathbb{Z}$ est transitive, il vient :

$$
\left(\Delta_{i} \times \mathbb{Z} \times Z_{I^{o}}\right) / \mathrm{GL}_{d}\left(F_{o}\right) \simeq\left(\Delta_{i} \times Z_{I^{o}}\right) / \mathrm{GL}_{d}^{\prime}\left(F_{o}\right) \simeq \bigsqcup Z_{I^{o}} / S^{\prime}\left(\delta_{j}\right)
$$

où l'on a choisi des représentants $\delta_{j}$ des différentes classes de $\Delta_{i}$ sous $\mathrm{GL}_{d}^{\prime}\left(F_{o}\right)$. Il suffit donc de montrer qu'un sous-groupe compact ouvert $H$ de $\mathrm{GL}_{d}\left(F_{o}\right)$ (typiquement $S^{\prime}\left(\delta_{j}\right)$ ) agit sans point fixe sur $Z_{I^{\circ}}$ (ce qui sera vrai sous l'unique hypothèse que $I^{o}$ est non vide).

Soit donc $z$ un représentant d'une double classe de $Z_{I^{o}}$; notant $z^{\prime}$ l'élément de $\bar{D}^{*}\left(\mathbb{A}^{\infty}\right)$ qui coincide avec $z$ en toute place $v \neq o$ mais dont la $o$-composante est 1 , le stabilisateur de $z^{\prime}$ est le sous-groupe $\Gamma_{z^{\prime}}=\bar{D}^{*}(F) \cap z^{\prime} K_{I}^{\infty, o} z^{\prime-1}$ de $\mathrm{GL}_{d}\left(F_{o}\right)$, où l'intersection est prise dans $\bar{D}^{*}\left(\mathbb{A}^{\infty, o}\right)$ puis, vue comme sous-groupe de $\bar{D}^{*}(F)$, injectée dans $\bar{D}^{*}\left(F_{o}\right)$. Le stabilisateur de $z$ est le conjugué $\Gamma_{z}=z_{o}^{-1} \Gamma_{z^{\prime}} z_{o}$. Puisque $H$ est compact et $\Gamma_{z}$ discret, l'intersection $H \cap \Gamma_{z}$ est finie ; elle est en fait triviale, car $\Gamma_{z}$ est sans torsion (cf. lemme précédent). Donc $H$ agit librement sur $Z_{I^{o}}$, ce qui démontre la première assertion.

Montrons le second point : supposons $\left(U_{n}(\delta g, r g) \times\{z g\}\right) \cap\left(U_{n}(\delta, r) \times\{z\}\right) \neq \emptyset$; alors $g$ laisse fixe $r$ et $z$, donc appartient à $\Gamma_{z} \cap \mathrm{GL}_{d}^{\prime}\left(F_{o}\right)$. De deux choses l'une : soit $g$ laisse fixe un sommet de l'immeuble, auquel cas il appartient à un sous-groupe ouvert compact $H$ et l'on conclut comme précédemment, soit il agit librement sur $\Delta_{0}$. Dans ce dernier cas, le résultat découle du lemme suivant :

Lemme 10.11. - Sig envoie un sommet s sur un sommet voisin alors val(det $g)$ n'est pas un multiple de $d$.

Démonstration. - Soit $L$ un réseau qui représente la classe de $s$ et $L^{\prime} \subset L$ le représentant de g.s tel que $L^{\prime} \not \subset \pi L$. D'après la théorie des facteurs invariants, il existe une $\mathcal{O}_{o}$-base $\epsilon=\left\{e_{1}, \ldots, e_{d}\right\}$ pour laquelle $L=\oplus \mathcal{O}_{o} e_{i}$ et $L^{\prime}=\oplus \pi^{r_{i}} \mathcal{O}_{o} e_{i}, 0=r_{1} \leq \ldots \leq r_{d}$. Puisque $s$ et $g . s$ sont deux sommets voisins, il existe $0<t<d$ tel que $r_{1}=\cdots=r_{t}=0$ et $r_{t+1}=\cdots=r_{d}=1$. Soit $a \in K^{*}$ tel que $g(L)=a L^{\prime}$; dans la base $\epsilon$, la matrice de $g$ s'écrit comme le produit $d h$ de la matrice diagonale $d=\operatorname{diag}\left(\pi^{r_{1}} a, \ldots, \pi^{r_{d}} a\right)$ et d'une matrice $h \in \mathrm{GL}_{d}\left(\mathcal{O}_{o}\right)$. Par conséquent, $\operatorname{val}(\operatorname{det} g) \equiv-t \quad(\bmod d)$, ce qui démontre l'assertion.

Les conséquences du fait 3 sont les suivantes : l'application $U_{n}\left(\delta^{\prime}, r^{\prime}\right) \times\left\{z^{\prime}\right\} \rightarrow U_{n}[\delta, r, z]$ obtenue en restreignant $p$ est un isomorphisme pour tout triplet $\left(\delta^{\prime}, r^{\prime}, z^{\prime}\right) \in[\delta, r, z]$ et l'ensemble $p^{-1}\left(U_{n}[\delta, r, z]\right)$ est l'union disjointe d'une infinité de copies de $U_{n}[\delta, r, z]$ que $\mathrm{GL}_{d}\left(F_{o}\right)$ permute 
sans point fixe. Notant $U_{n}^{\prime}(i)=\bigsqcup_{[\delta, r, z] \in O_{i}} U_{n}[\delta, r, z]$, où $O_{i}$ désigne l'ensemble des orbites de $\Delta_{i} \times \mathbb{Z} \times Z_{I^{o}}$ sous $\mathrm{GL}_{d}\left(F_{o}\right)$, et $\widetilde{U}_{n}(i)=p^{-1}\left(U_{n}^{\prime}(i)\right)=U_{n}(i) \times Z_{I^{o}}$, le revêtement

$$
\widetilde{U}_{n}(i) \stackrel{p}{\longrightarrow} U_{n}^{\prime}(i) \simeq \widetilde{U}_{n}(i) / \mathrm{GL}_{d}\left(F_{o}\right)
$$

est donc le revêtement trivial.

Considérons la suite spectrale de Leray-Serre associée à ce revêtement de groupe $G=\mathrm{GL}_{d}\left(F_{o}\right)$ : elle provient de l'égalité suivante dans la catégorie dérivée des $\mathbb{Q}_{\ell}$-espaces vectoriels :

$$
R_{G} R \Gamma\left(\widetilde{U}_{n}(i), \mathcal{F}\right)=R \Gamma\left(U_{n}^{\prime}(i), p_{*} \mathcal{F}\right),
$$

pour tout $\mathbb{Q}_{\ell}$-module étale $\mathcal{F}$ sur $\widetilde{U}_{n}(i)$, et où $R_{G}$ désigne le foncteur dérivé de $M \mapsto M^{G}$. Comme il n'y a pas de $G$-cohomologie (excepté en degré 0 ), cette égalité se réécrit (prenant $\mathcal{F}=\mathbb{Q}_{\ell}$ et étendant les scalaires à $\left.\bar{F}_{o}\right)$ :

$$
R \Gamma\left(U_{n}^{\prime}(i)_{\bar{F}_{o}}, \mathbb{Q}_{\ell}\right)=R \Gamma\left(\widetilde{U}_{n}(i)_{\bar{F}_{o}}, \mathbb{Q}_{\ell}\right)^{\mathrm{GL}_{d}\left(F_{o}\right)} .
$$

10.2.4. calcul de $\mathrm{H}^{\cdot}\left(\mathcal{E} \ell \ell_{I} \otimes \bar{F}_{o}, \mathbb{Q}_{\ell}\right)$. - On dispose du recouvrement de Čech fini $\mathcal{U}^{\prime}=\left\{U_{n}[\delta, r, z]\right\}$ de l'espace analytique $\mathcal{E} \ell \ell_{I}^{a n}$. Considérons à nouveau la famille de complexes $T^{\cdot}=\left\{T_{m}\right\}$ du lemme A.14 de l'appendice. Notant $p r$ la projection $\operatorname{Res}^{\prime} \Sigma_{n}^{d} \times Z_{I^{o}} \longrightarrow \operatorname{Res}^{\prime} \Sigma_{n}^{d}$, puis définissant $T_{m}^{\prime j}=p_{*} p r^{*}\left(T_{m}^{j}\right)$, on vérifie facilement que $T_{m}^{\prime}$ est pour tout $m \geq 0$ une résolution $R \Gamma$-acyclique du faisceau constant $\mathbb{Z} / \ell^{m} \mathbb{Z}$ sur $\mathcal{E} \ell \ell_{I}$ (car le revêtement $p$ est trivial au-dessus de chaque $\left.U_{n}[\delta, r, z]\right)$. De plus, $\left\{T_{m}^{\prime}\right\}$ est pour tout $j \geq 0$ un faisceau $\ell$-adique lisse (au sens du paragraphe A.1). On forme alors le double complexe de Čech $\check{C}^{\cdot}\left(\mathcal{U}^{\prime}, T^{\prime}\right)$ défini par

$$
\check{C}^{i}\left(\mathcal{U}^{\prime}, T^{\prime j}\right):={\underset{\iota}{m}}_{\lim } \Gamma\left(U_{n}^{\prime}(i)_{\bar{F}_{o}}, T_{m}^{\prime j}\right) \otimes_{\mathbb{Z}_{\ell}} \mathbb{Q}_{\ell} ;
$$

il vérifie l'égalité suivante dans la catégorie dérivée des $\mathbb{Q}_{\ell}$-espaces vectoriels (cf. [Go] §5.3) :

$$
\operatorname{Tot}\left(\check{C}^{\cdot}\left(\mathcal{U}^{\prime}, T^{\prime}\right)\right)=R \Gamma\left(\mathcal{E} \ell \ell_{I} \otimes \overline{F_{o}}, \mathbb{Q}_{\ell}\right) .
$$

Désignons par $\mathcal{A}_{I^{o}}^{\infty}$ l'espace des formes automorphes sur $Z_{I^{o}}$ triviales à l'infini. Nous allons voir qu'il existe des quasi-isomorphismes canoniques (lorsque $i$ varie)

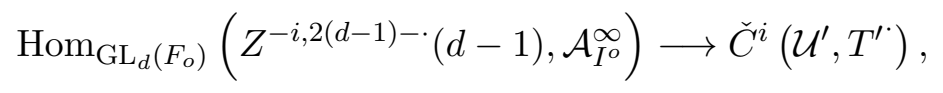

où l'action de $\mathrm{GL}_{d}\left(F_{o}\right)$ sur $Z$,' est celle du paragraphe 10.2.1 composée avec l'application $g \mapsto$ ${ }^{t} g^{-1}$. Notant $\mathcal{P} \cdot\left(\mathcal{E} \ell \ell_{I}\right)$ le complexe total associé au double complexe du membre de gauche, c'est-à-dire

$$
\mathcal{P} \cdot\left(\mathcal{E} \ell \ell_{I}\right)=\operatorname{Hom}_{\mathrm{GL}_{d}\left(F_{o}\right)}\left(\mathcal{P}^{2(d-1)-\cdot}(d-1), \mathcal{A}_{I^{o}}^{\infty}\right),
$$

on aura ainsi démontré :

Fait 4. - On a des isomorphismes $\mathrm{GL}_{d}\left(F_{o}\right)$-équivariants (pour l'action mentionnée plus haut) :

$$
h^{j}\left(\mathcal{P} \cdot\left(\mathcal{E} \ell \ell_{I}\right)\right) \stackrel{\sim}{\rightarrow} \mathrm{H}^{j}\left(\mathcal{E} \ell \ell_{I} \otimes \bar{F}_{o}, \mathbb{Q}_{\ell}\right) \quad \forall j \geq 0 .
$$

Partons des quasi-isomorphismes (4) ; le complexe $\check{C}_{c}^{i}\left(\mathcal{U}, T^{*}\right)$ représente $R \Gamma\left(U_{n}(i), \mathbb{Q}_{\ell}\right)$ (vu dans la catégorie dérivée des $\mathbb{Q}_{\ell}-\mathrm{GL}_{d}\left(F_{o}\right)$-modules). Appliquant le théorème de dualité de Poincaré, établi par Berkovich (cf. théorème A.6), on obtient des quasi-isomorphismes canoniques

$$
\operatorname{Hom}_{\mathbb{Q}_{\ell}}\left(Z^{-i, 2(d-1)-\cdot}(d-1), \mathbb{Q}_{\ell}\right) \longrightarrow \Gamma\left(U_{n}(i), T^{\cdot}\right),
$$


où, par définition, $\Gamma\left(U_{n}(i), T^{j}\right):=\lim _{m} \Gamma\left(U_{n}(i), T_{m}^{j}\right) \otimes_{\mathbb{Z}_{\ell}} \mathbb{Q}_{\ell}$ (on pourrait également noter $\left.\Gamma\left(U_{n}(i), T^{*}\right)=\check{C}^{i}\left(\mathcal{U}, T^{\cdot}\right)\right)$. On utilise ensuite l'égalité suivante (toujours dans la catégorie dérivée $\operatorname{des} \mathbb{Q}_{\ell}-\mathrm{GL}_{d}\left(F_{o}\right)$-modules) :

$$
R \Gamma\left(U_{n}(i) \times Z_{I^{o}}, \mathbb{Q}_{\ell}\right)=\operatorname{Hom}\left(Z_{I^{o}}, R \Gamma\left(U_{n}(i), \mathbb{Q}_{\ell}\right)\right)
$$

$\left(Z_{I^{o}}\right.$ est un ensemble discret). Il en résulte des quasi-isomorphismes $\mathrm{GL}_{d}\left(F_{o}\right)$-équivariants canoniques (lorsque $i$ varie) :

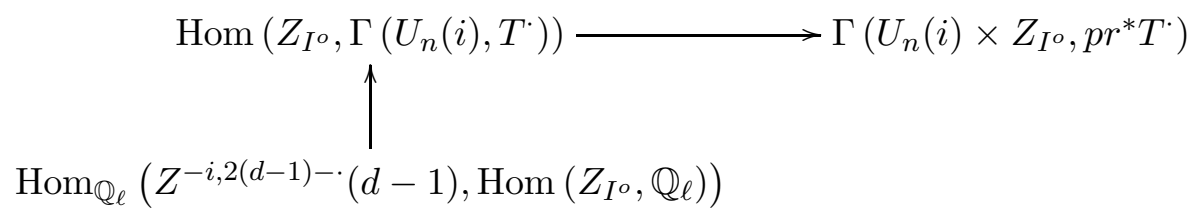

où, par définition, $\Gamma\left(U_{n}(i) \times Z_{I^{o}}, p r^{*} T^{j}\right):=\varliminf_{m} \Gamma\left(U_{n}(i) \times Z_{I^{o}}, p r^{*} T_{m}^{j}\right) \otimes_{\mathbb{Z}_{\ell}} \mathbb{Q}_{\ell}$. Finalement, on utilise l'égalité (6); on obtient ainsi les quasi-isomorphismes (7) annoncés, en prenant les invariants sous l'action de $\mathrm{GL}_{d}\left(F_{o}\right)$, sachant que l'image de $f \in \operatorname{Hom}\left(Z^{-i, 2(d-1)-\cdot}(d-1), \mathcal{A}_{I^{o}}^{\infty}\right)$ par $g \in \mathrm{GL}_{d}\left(F_{o}\right)$ est $z \mapsto g \cdot f\left({ }^{t} g \cdot z\right)$.

10.2.5. suite et fin. - Considérons le foncteur $F=R \operatorname{Hom}_{\mathrm{GL}_{d}\left(F_{o}\right)}\left(\bullet, \mathcal{A}_{I^{o}}^{\infty}\right)$ défini sur la catégorie dérivée des $\mathbb{Q}_{\ell}-\mathrm{GL}_{d}\left(F_{o}\right)$-modules lisses. Nous disposons de deux suites spectrales, relativement au foncteur $F$ (cf [EGA3] chap. 0 11.4.3) :

$$
\begin{aligned}
& { }^{\prime} \mathrm{E}_{2}^{i, j}=h^{i}\left(R^{j} F\left(C^{\cdot}\right)\right) \Longrightarrow \mathbb{R}^{i+j} F\left(C^{\cdot}\right) \\
& { }^{\prime \prime} \mathrm{E}_{2}^{i, j}=R^{i} F\left(h^{j}\left(C^{\cdot}\right)\right) \Longrightarrow \mathbb{R}^{i+j} F\left(C^{\cdot}\right),
\end{aligned}
$$

où $\mathbb{R}^{k} F$ désigne le foncteur hyperdérivé. Prenant $C^{\cdot}=\mathcal{P}^{2(d-1)-\cdot}(d-1)$, qui est un complexe projectif (de sorte que $R^{i} F\left(C^{\cdot}\right)=0$ pour tout $i>0$ ), la première suite spectrale est dégénérée (cf. loc. cit. prop. 11.4.5). La seconde suite spectrale se réécrit donc :

$$
\mathrm{E}_{2}^{i, j}=R^{i} F\left(h^{2(d-1)-j}\left(\mathcal{P}^{\cdot}\right)(d-1)\right) \Longrightarrow h^{i+j}\left(F\left(\mathcal{P}^{2(d-1)-\cdot}(d-1)\right)\right) .
$$

C'est exactement la suite spectrale de la proposition 10.6, puisque $R^{i} F=\operatorname{Ext}_{\mathrm{GL}_{d}\left(F_{o}\right)}^{i}\left(\bullet, \mathcal{A}_{I^{o}}^{\infty}\right)$ et l'on sait que :

$$
\begin{aligned}
h^{k}(\mathcal{P}) & \stackrel{\sim}{\rightarrow} \mathrm{H}_{c}^{k}\left(\left(\operatorname{Res}^{\prime} \Sigma_{n}^{d}\right)_{\bar{F}_{o}}, \mathbb{Q}_{\ell}\right), \\
h^{k}\left(F\left(\mathcal{P}^{2(d-1)-\cdot}(d-1)\right)\right) & \stackrel{\sim}{\rightarrow} \mathrm{H}^{k}\left(\mathcal{E} \ell \ell_{I} \otimes \bar{F}_{o}, \mathbb{Q}_{\ell}\right) .
\end{aligned}
$$

Enfin, d'après le théorème de comparaison établi par Berkovich (cf. th. A.4), $\mathrm{H}^{\cdot}\left(\mathcal{E} \ell \ell_{I} \otimes \bar{F}_{o}, \mathbb{Q}_{\ell}\right)$ désigne indifféremment la cohomologie des espaces analytiques ou la cohomologie étale habituelle.

10.3. Dégénérescence de la partie cuspidale de la suite spectrale. - On se propose de démontrer dans ce paragraphe la dégénérescence de la partie cuspidale de la suite spectrale figurant dans l'énoncé du corollaire 10.7.

On poursuit avec les notations précédentes. Soit $\pi_{o}$ un élément de $\mathcal{A}_{d}^{0}\left(F_{o}\right)$, de caractère central $\xi_{o}$ d'ordre fini. Appliquant le lemme 10.2, on regarde $\pi_{o}$ comme la composante en $o$ d'une représentation automorphe $\widetilde{\Pi}$ de $\mathrm{GL}_{d}(\mathbb{A})$ vérifiant les conditions du lemme. Puis on transfère $\widetilde{\Pi}$ aux groupes $D^{*}(\mathbb{A})$ et $\bar{D}^{*}(\mathbb{A})$ (cf. lemme 10.3 ou théorème 10.4 2.) : on obtient finalement des représentations automorphes $\Pi$ et $\bar{\Pi}$ respectivement, de multiplicité un, telles que

$$
\begin{aligned}
& \Pi_{o} \simeq \mathrm{JL}\left(\pi_{o}\right), \quad \Pi_{o^{\prime}} \simeq \operatorname{JL}\left(\widetilde{\Pi}_{o^{\prime}}\right), \text { et } \Pi^{o, o^{\prime}} \simeq \widetilde{\Pi}^{o, o^{\prime}} ; \\
& \bar{\Pi}_{\infty} \simeq \mathrm{JL}\left(\widetilde{\Pi}_{\infty}\right)=1_{\infty}, \quad \bar{\Pi}_{o^{\prime}} \simeq \operatorname{JL}\left(\widetilde{\Pi}_{o^{\prime}}\right) \text {, et } \bar{\Pi}^{\infty, o^{\prime}} \simeq \widetilde{\Pi}^{\infty, o^{\prime}} \text {. }
\end{aligned}
$$


On voit que $\bar{\Pi}^{\infty, o} \simeq \Pi^{\infty, o}$; de plus, $\bar{\Pi}$ est l'unique (à isomorphisme près) représentation automorphe $\nu$ de $\bar{D}^{*}(\mathbb{A})$ vérifiant $\nu^{\infty, o} \simeq \Pi^{\infty, o}$ (une telle représentation $\nu$ cö̈ncide avec $\widetilde{\Pi}$ en dehors de $S=\left\{\infty, o, o^{\prime}\right\}$; on applique le théorème 10.4 2.). Notant $W\left[\Pi^{\infty, o}\right]$ la composante $\Pi^{\infty, o}$-isotypique pour toute représentation semi-simple $W$ de $D^{*}\left(\mathbb{A}^{\infty, o}\right) \simeq \bar{D}^{*}\left(\mathbb{A}^{\infty, o}\right)$, on a donc :

$$
\left(\left(\mathcal{A} \frac{\infty}{D}\right)\left[\Pi^{\infty, o}\right]\right)_{o} \simeq \pi_{o}
$$

comme représentation de $\bar{D}^{*}\left(F_{o}\right) \simeq \mathrm{GL}_{d}\left(F_{o}\right)$.

Revenons à la suite spectrale du corollaire 10.7. On considère à partir de maintenant la cohomologie étale à coefficients dans $\overline{\mathbb{Q}}_{\ell}$ (qui s'obtient à partir de $\mathrm{H}^{\cdot}\left(\bullet, \mathbb{Q}_{\ell}\right.$ ) par extension des coefficients). Notre suite spectrale s'écrit :

$$
\mathrm{E}_{2}^{i, j}=\operatorname{Ext}_{\mathrm{GL}_{d}\left(F_{o}\right)-\text { mod. lisse }}^{i}\left(\mathrm{H}_{c}^{2(d-1)-j}\left(\left(\operatorname{Res}^{\prime} \Sigma_{n}^{d}\right)_{\bar{F}_{o}}, \overline{\mathbb{Q}}_{\ell}\right)(d-1), \mathcal{A}_{\bar{D}}^{\infty}\right) \Longrightarrow H_{o, n}^{i+j},
$$

où l'on note $H_{o, n}^{k}=\mathrm{H}^{k}\left(\mathcal{E} \ell \ell_{n}^{\infty} \otimes \bar{F}_{o}, \overline{\mathbb{Q}}_{\ell}\right)$. Elle est équivariante pour l'action de $D^{*}\left(\mathbb{A}^{\infty}\right) \times W_{F_{o}} \simeq$ $\bar{D}^{*}\left(\mathbb{A}^{\infty, o}\right) \times D_{o}^{*} \times W_{F_{o}}$.

Pour toute représentation $V$ admissible (au sens du début du paragraphe 9.3, en prenant $\left.H=D^{*}\left(\mathbb{A}^{\infty}\right)\right)$ du groupe produit $D^{*}\left(\mathbb{A}^{\infty}\right) \times W_{F_{o}}$, toute représentation admissible irréductible $\tau_{o}$ de $D_{o}^{*}$ et toute représentation irréductible $\sigma_{o}$ de $W_{F_{o}}$, nous notons $m_{\bar{\Pi}^{\infty, o} \otimes \tau_{o} \otimes \sigma_{o}}(V)$ la multiplicité de $\bar{\Pi}^{\infty, o} \otimes \tau_{o} \otimes \sigma_{o}$ dans $V$ (le lecteur pourra consulter Boy] $\S 3.1$, par exemple, pour des rappels concernant la notion de multiplicité dans un tel contexte).

Lemme 10.12. - $E_{2}^{i, j}$ est admissible en tant que représentation de $D^{*}\left(\mathbb{A}^{\infty}\right) \times W_{F_{o}}$.

Démonstration. - Comme $\mathrm{H}_{c}^{\bullet}\left(\Sigma_{n}^{d}\right):=\mathrm{H}_{c}^{\bullet}\left(\left(\left(\operatorname{Res}^{\prime} \Sigma_{n}^{d}\right)_{\bar{F}_{o}}, \overline{\mathbb{Q}}_{\ell}\right)\right.$ est un $\mathrm{GL}_{d}\left(F_{o}\right)$-module de type fini (cf. proposition 10.6 (i)), les Ext se calculent en écrivant une résolution projective de $\mathrm{H}_{c}^{\bullet}\left(\Sigma_{n}^{d}\right)$ par des sommes finies de termes du type c-Ind $G_{G^{\prime}}^{G} \pi$, où $G^{\prime}$ est un sous-groupe compact ouvert de $G:=\mathrm{GL}_{d}\left(F_{o}\right)$ et $\pi$ une représentation de dimension finie de $G^{\prime}$.

Soit maintenant $K^{o}$ un sous-groupe compact ouvert de $D^{*}\left(\mathbb{A}^{\infty, o}\right)$. On a :

$$
\operatorname{hom}_{G}\left(\mathrm{c}-\operatorname{Ind}_{G^{\prime}}^{G} \pi,\left(\mathcal{A} \frac{\infty}{D}\right)^{K^{o}}\right) \simeq \operatorname{hom}_{G^{\prime}}\left(\pi,\left(\mathcal{A}_{\bar{D}}^{\infty}\right)^{K^{o}}\right)=\operatorname{hom}_{G^{\prime}}\left(\pi,\left(\mathcal{A}_{\bar{D}}^{\infty}\right)^{K^{\circ} K^{\prime}}\right)
$$

où $K^{\prime} \subset G$ est un sous-groupe ouvert compact tel que $\pi_{\mid K^{\prime}}=$ Id (le premier isomorphisme résulte de la dualité de Frobenius, cf. [B-Z $]$ prop. 2.29). Comme $\left(\mathcal{A} \frac{\infty}{D}\right)^{K^{\circ} K^{\prime}}$ est de dimension finie en vertu de l'admissibilité de l'espace des formes automorphes, le calcul des Ext ne fait intervenir qu'un nombre fini de représentations automorphes П. Par conséquent,

$$
\operatorname{Ext}_{G-\text { mod. lisse }}^{i}\left(\mathrm{H}_{c}^{\bullet}\left(\Sigma_{n}^{d}\right), \mathcal{A}_{\bar{D}}^{\infty}\right)^{K^{o}} \simeq \bigoplus_{\Pi_{\infty}=1_{\infty}} \operatorname{Ext}_{G-\text { mod. lisse }}^{i}\left(\mathrm{H}_{c}^{\bullet}\left(\Sigma_{n}^{d}\right), \Pi_{o}\right) \otimes\left(\Pi^{o}\right)^{K^{o}}
$$

est de dimension finie. En effet, $\operatorname{dim}_{\overline{\mathbb{Q}}_{\ell}} \operatorname{Ext}_{G-\bmod \text { lisse }}^{i}\left(\mathrm{H}_{c}^{\bullet}\left(\Sigma_{n}^{d}\right), \Pi_{o}\right)<\infty$ en vertu du lemme suivant.

Lemme 10.13 (cf. $\mathbf{F a r}$ lemme 2.6.15). - Soit $G$ un groupe p-adique réductif, $\pi_{1}$ une représentation lisse de type fini de $G$ et $\pi_{2}$ une représentation admissible. Alors

$$
\operatorname{dim}\left(\operatorname{Ext}_{G-\text { mod. lisse }}^{i}\left(\pi_{1}, \pi_{2}\right)\right)<\infty \quad \forall i .
$$

Démonstration. - On construit une résolution projective de $\pi_{1}$ par des représentations de type fini de $G$ (utilise la locale noethérianité de la catégorie des représentations lisses de $G$, cf. D-K-V Remarque 3.12). On conclut par le fait suivant : si $\rho$ est de type fini et $\pi_{2}$ est admissible, alors $\operatorname{hom}_{G}\left(\rho, \pi_{2}\right)$ est de dimension finie. 
Nous pouvons maintenant considérer les multiplicités $m_{\bar{\Pi}^{\infty, o} \otimes \tau_{o} \otimes \sigma_{o}}\left(E_{2}^{i, j}\right)$.

Lemme 10.14. - On a $m_{\bar{\Pi}^{\infty, o} \otimes \tau_{o} \otimes \sigma_{o}}\left(E_{2}^{i, j}\right)=0 \quad \forall i>0$.

Démonstration. - Nous allons montrer que les groupes

$$
\operatorname{Ext}_{\mathrm{GL}_{d}\left(F_{o}\right)-\text { mod. lisse }}^{i}\left(\mathrm{H}_{c}^{2(d-1)-j}\left(\left(\operatorname{Res}^{\prime} \Sigma_{n}^{d}\right)_{\bar{F}_{o}}, \overline{\mathbb{Q}}_{\ell}\right)(d-1), \pi_{o}\right)
$$

sont nuls en dimension $i>0$; étant donné la relation (8), le lemme en résultera. Rappelons que $\operatorname{Res}^{\prime} \Sigma_{n}^{d}$ est l'union disjointe de copies, indexées par $\mathbb{Z}$, de l'espace de Drinfeld $\Sigma_{n}^{d}$. Le stabilisateur d'une composante connexe, pour l'action de $\mathrm{GL}_{d}\left(F_{o}\right)$, est le sous-groupe $\mathrm{GL}_{d}^{\prime}\left(F_{o}\right)$ constitué des éléments dont le déterminant est une unité de l'anneau des entiers $\mathcal{O}_{o}$. En vertu de la dualité de Frobenius, on dispose d'un isomorphisme de foncteurs :

$$
\begin{array}{r}
\operatorname{Hom}_{\mathrm{GL}_{d}\left(F_{o}\right)}\left(\mathrm{H}_{c}^{j}\left(\left(\operatorname{Res}^{\prime} \Sigma_{n}^{d}\right)_{\bar{F}_{o}}, \overline{\mathbb{Q}}_{\ell}\right)(d-1), \bullet\right) \\
\simeq \operatorname{Hom}_{\mathrm{GL}_{d}^{\prime}\left(F_{o}\right)}\left(\mathrm{H}_{c}^{j}\left(\left(\Sigma_{n}^{d}\right)_{\bar{F}_{o}}, \overline{\mathbb{Q}}_{\ell}\right)(d-1), \operatorname{Res}_{\mathrm{GL}_{d}^{\prime}\left(F_{o}\right)}^{\mathrm{GL}_{d}\left(F_{0}\right)} \bullet .\right.
\end{array}
$$

Il en résulte des isomorphismes

$$
\begin{array}{r}
\operatorname{Ext}_{\mathrm{GL}_{d}\left(F_{o}\right)-\text { mod. lisse }}^{i}\left(\mathrm{H}_{c}^{2(d-1)-j}\left(\left(\operatorname{Res}^{\prime} \Sigma_{n}^{d}\right)_{\bar{F}_{o}}, \overline{\mathbb{Q}}_{\ell}\right)(d-1), \pi_{o}\right) \\
\stackrel{\sim}{\rightarrow} \operatorname{Ext}_{\mathrm{GL}_{d}^{\prime}\left(F_{o}\right)-\text { mod. lisse }}^{i}\left(\mathrm{H}_{c}^{2(d-1)-j}\left(\left(\Sigma_{n}^{d}\right)_{\bar{F}_{o}}, \overline{\mathbb{Q}}_{\ell}\right)(d-1), \operatorname{Res}_{\mathrm{GL}_{d}^{\prime}\left(F_{o}\right)}^{\left.\mathrm{G}_{o}\right)} \pi_{o}\right) .
\end{array}
$$

Par ailleurs, d'après le théorème de Harish-Chandra (voir [ $\mathbf{B}-\mathbf{Z}$, théorème 3.21) appliqué à la représentation supercuspidale $\pi_{o}$, la restriction $\operatorname{Res}_{\mathrm{GL}_{d}^{\prime}\left(F_{o}\right)}^{\mathrm{GL}_{d}\left(F_{o}\right)} \pi_{o}$ est une représentation finie (au sens de [B-Z 2.40 : les coefficients matriciels sont des fonctions à support compact). Le résultat découle alors de l'isomorphisme (10) et du lemme suivant.

Lemme 10.15 (d'après Bernstein-Zelevinski). - Soit $(\rho, V)$ une représentation finie d'un groupe topologique $G$ (unimodulaire, dénombrable à l'infini et tel qu'il existe un système fondamental de voisinages de l'élément neutre formé de sous-groupes compacts ouverts). Alors $(\rho, V)$ est un objet projectif dans la catégorie des représentations lisses de $G$.

Démonstration. - D'après le théorème 2.44 dans $[\mathbf{B}-\mathbf{Z}]$, toute représentation finie est complètement réductible; on peut donc supposer $V$ irréductible. On se donne alors un diagramme

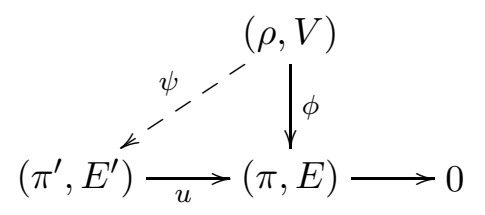

et il s'agit de relever $\phi$ en un morphisme $\psi$. La clef est encore dans la preuve du théorème 2.44 de Bernstein et Zelevinski : ces derniers définissent une projection $p_{\pi}: E \rightarrow E$ (notée $\pi\left(\varepsilon^{\rho}\right)$ dans loc. cit.) qui commute à l'action de $G$ et tel que, considérant également $p_{\pi^{\prime}}: E^{\prime} \rightarrow E^{\prime}$ et tout $f \in \operatorname{Hom}_{G}\left(\pi^{\prime}, \pi\right)$ (par exemple $f=u$ ), on ait $f \circ p_{\pi^{\prime}}=p_{\pi} \circ f$. De plus, im $p_{\pi}$ (donc également $\left.\operatorname{im} p_{\pi^{\prime}}\right)$ se scinde en une somme directe de $G$-représentations isomorphes à $V$.

Soit $0 \neq v \in V$; puisque $V$ est irréductible, il suffit de définir $\psi$ sur des combinaisons linéaires finies $\sum_{i} \lambda_{i} \rho\left(g_{i}\right) v$. Choisissons un relèvement quelconque $w$ de $\phi(v)$ dans $\operatorname{im} p_{\pi^{\prime}}$. On définit alors : $\psi\left(\sum_{i} \lambda_{i} \rho\left(g_{i}\right) v\right)=\sum_{i} \lambda_{i} \pi^{\prime}\left(g_{i}\right) w$. Cela a un sens, à condition qu'une somme $\sum_{i} \lambda_{i} \rho\left(g_{i}\right) v=0$ donne une image nulle, autrement dit (appliquant $\phi$ ), si un élément $\sum_{i} \lambda_{i} \pi^{\prime}\left(g_{i}\right) w$ du noyau de $u$ est 
le vecteur nul. Cela résulte de l'observation suivante : $\operatorname{ker} u \cap \operatorname{im} p_{\pi^{\prime}}=\{0\}$. En effet, puisque $u$ commute à $p_{\pi}$ et $p_{\pi^{\prime}}$, alors $u$ envoie $\operatorname{ker} p_{\pi}\left(\operatorname{resp}\right.$. im $\left.p_{\pi}\right)$ dans ker $p_{\pi^{\prime}}\left(\right.$ resp. im $\left.p_{\pi^{\prime}}\right)$. Supposons que $x=p_{\pi^{\prime}}(y)$ appartienne au noyau de $u$; on a $p_{\pi}(u(y))=u(x)=0$, donc $y$ appartient au noyau de $p_{\pi^{\prime}}$ et $x=0$.

Remarque 10.16. - Conservons les hypothèses du lemme précédent et supposons de plus que $(\rho, V)$ soit irréductible. Alors $(\rho, V)$ est également injective dans la catégorie des représentations lisses de $G$.

En effet, il s'agit de compléter le diagramme suivant :

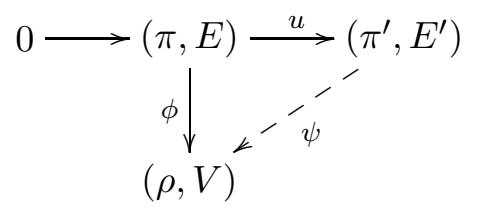

Ecrivant $E=\operatorname{ker} p_{\pi} \oplus \operatorname{im} p_{\pi}$ et $E^{\prime}=\operatorname{ker} p_{\pi^{\prime}} \oplus \operatorname{im} p_{\pi^{\prime}}$, on sait que $\operatorname{im} p_{\pi}$ et $\operatorname{im} p_{\pi^{\prime}}$ se scindent en une somme directe de copies de $V$, alors que les représentations ker $p_{\pi}$ et $\operatorname{ker} p_{\pi^{\prime}}$ ne contiennent aucun sous-facteur isomorphe à $V$. Nécessairement, $\left.\phi\right|_{\operatorname{ker} p_{\pi}}=0$; de plus, $u$ respecte les décompositions précédentes. Il est alors clair que l'on peut construire un morphisme $\psi$.

Proposition 10.17. - Pour tout entier $0 \leq j \leq 2(d-1)$,

$$
E_{2}^{0, j}\left[\Pi^{\infty, o}\right]=\operatorname{Hom}_{\mathrm{GL}_{d}\left(F_{o}\right)}\left(\mathrm{H}_{c}^{2(d-1)-j}\left(\left(\operatorname{Res}^{\prime} \Sigma_{n}^{d}\right)_{\bar{F}_{o}}, \overline{\mathbb{Q}}_{\ell}\right)(d-1), \pi_{o}\right) \stackrel{\sim}{\longrightarrow}\left(\left(H_{o, n}^{j}\right)^{s s}\left[\Pi^{\infty, o}\right]\right)_{o}
$$

(isomorphismes entre représentations de $D_{o}^{*} \times W_{F_{o}}$ ).

Cette proposition signifie que la partie cuspidale de la suite spectrale (9) est dégénérée. Cela provient essentiellement du lemme précédent 10.14, en ajoutant un argument de comptage des multiplicités :

Démonstration. - La multiplicité $m_{\bar{\Pi}^{\infty, o} \otimes \tau_{o} \otimes \sigma_{o}}(\bullet)$ est additive sur les suites exactes courtes et est positive sur les représentations effectives, de sorte qui si une représentation admissible $V$ de $D^{*}\left(\mathbb{A}^{\infty}\right) \times W_{F_{o}}$ est telle que $m_{\bar{\Pi}^{\infty, o} \otimes \tau_{o} \otimes \sigma_{o}}(V)=0$, alors il en est de même pour tout sous-quotient de $V$. Comme $E_{r}^{i, j}$ est un sous-quotient de $E_{r-1}^{i, j}$, on déduit alors du lemme 10.14, par récurrence sur $r$, que $m_{\bar{\Pi}^{\infty, o} \otimes \tau_{o} \otimes \sigma_{o}}\left(E_{r}^{i, j}\right)=0$ pour tout $i \neq 0$, tout $j$ et tout $r \geq 2$.

Nous allons montrer que $m_{\bar{\Pi}^{\infty, o} \otimes \tau_{o} \otimes \sigma_{o}}\left(E_{2}^{i, j}\right)=m_{\bar{\Pi}^{\infty, o} \otimes \tau_{o} \otimes \sigma_{o}}\left(E_{\infty}^{i, j}\right) \quad \forall i, j$; pour cela, nous avons besoin d'un lemme :

Lemme 10.18 (cf. Boy lemme 13.3.2). - Soient $U, V$ et $W$ trois représentations admissibles de $D^{*}\left(\mathbb{A}^{\infty}\right) \times W_{F_{o}}$ et $f: U \rightarrow V, g: V \rightarrow W$ deux morphismes équivariants. On suppose que les multiplicités $m_{\bar{\Pi}^{\infty, o} \otimes \tau_{o} \otimes \sigma_{o}}(U)$ et $m_{\bar{\Pi}^{\infty, o} \otimes \tau_{o} \otimes \sigma_{o}}(W)$ sont nulles. Alors on a l'égalité :

$$
m_{\bar{\Pi}^{\infty, o} \otimes \tau_{o} \otimes \sigma_{o}}(V)=m_{\bar{\Pi}^{\infty, o} \otimes \tau_{o} \otimes \sigma_{o}}(\operatorname{ker} g / \operatorname{im} f) .
$$

Procédons par récurrence sur $r \geq 2:$ si $i=r$, alors $E_{r}^{i, j}$ et $E_{r+1}^{i, j}$ sont tous deux de multiplicité nulle. Dans le cas contraire, on applique le lemme aux différentielles $d_{r}^{i, j}: E_{r}^{i, j} \rightarrow E_{r}^{i+r, j+r-1}$ et $d_{r}^{p-r, j-r+1}: E_{r}^{i-r, j-r+1} \rightarrow E_{r}^{i, j}:$ on obtient

$$
m_{\bar{\Pi}^{\infty, o} \otimes \tau_{o} \otimes \sigma_{o}}\left(E_{r+1}^{i, j}\right)=m_{\bar{\Pi}^{\infty, o} \otimes \tau_{o} \otimes \sigma_{o}}\left(\operatorname{ker} d_{r}^{i, j} / \operatorname{im} d_{r}^{p-r, j-r+1}\right)=m_{\bar{\Pi}^{\infty, o} \otimes \tau_{o} \otimes \sigma_{o}}\left(E_{r}^{i, j}\right) .
$$

Finalement, puisque la suite $\left(E_{r}^{i, j}\right)_{r}$ est stationnaire à partir d'un certain rang, il vient l'égalité annoncée $m_{\bar{\Pi}^{\infty, o} \otimes \tau_{o} \otimes \sigma_{o}}\left(E_{2}^{i, j}\right)=m_{\bar{\Pi}^{\infty, o} \otimes \tau_{o} \otimes \sigma_{o}}\left(E_{\infty}^{i, j}\right)$. 
On conclut facilement : comme $E_{\infty}^{n}$ est filtré par les $E_{\infty}^{i, j}$ pour $i+j=n$ et que les multiplicités précédentes sont nulles sauf pour $i=0$, on a l'égalité $m_{\bar{\Pi}^{\infty, o} \otimes \tau_{o} \otimes \sigma_{o}}\left(E_{2}^{0, j}\right)=m_{\bar{\Pi}^{\infty, o} \otimes \tau_{o} \otimes \sigma_{o}}\left(E_{\infty}^{j}\right)$. C'est vrai pour tout couple $\left(\tau_{o}, \sigma_{o}\right)$, d'où l'assertion de la proposition.

10.4. Fin de la preuve. - Après passage à la limite projective sur $n$, les isomorphismes de la proposition 10.17 s'écrivent :

$$
\operatorname{Hom}_{\mathrm{GL}_{d}\left(F_{o}\right)}\left(\Psi_{d}^{2(d-1)-j}(d-1), \pi_{o}^{\vee}\right) \stackrel{\sim}{\longrightarrow}\left(\left(H_{o}^{j}\right)^{s s}\left[\Pi^{\infty, o}\right]\right)_{o}
$$

(avec les notations des paragraphes 9.2 et 10.1). L'apparition de la contragrédiente $\pi_{o}^{\vee}$ provient de la remarque 10.8. D'autre part, il est clair que $\operatorname{Hom}_{\mathrm{GL}_{d}\left(F_{o}\right)}\left(\Psi_{d}^{2(d-1)-j}(d-1)\left(\xi_{o}\right), \pi_{o}^{\vee}\right)$ et $\operatorname{Hom}_{\mathrm{GL}_{d}\left(F_{o}\right)}\left(\Psi_{d}^{2(d-1)-j}(d-1), \pi_{o}^{\vee}\right)$ s'identifient naturellement; on a donc, pour tout entier $0 \leq j \leq 2(d-1):$

$$
\operatorname{Hom}_{\mathrm{GL}_{d}\left(F_{o}\right)}\left(\Psi_{d}^{2(d-1)-j}(d-1)\left(\xi_{o}\right), \pi_{o}^{\vee}\right) \simeq\left(\left(H_{o}^{j}\right)^{s s}\left[\Pi^{\infty, o}\right]\right)_{o}
$$

(isomorphisme entre représentations de $D_{o}^{*} \times W_{F_{o}}$ ).

Récapitulons où nous en sommes : partant de $\pi_{o} \in \mathcal{A}_{d}^{0}(K)$, pour $K$ un corps local d'égale caractéristique $p$, on s'est donné un corps global $F$ tel que le complété $F_{o}$ en $o$ soit isomorphe à $K$. Le passage du local au global, via le théorème d'uniformisation de Čerednik-Drinfeld, a permis de relier les représentations $\Psi_{d}^{i}\left(\xi_{o}\right)$ aux représentations $H_{o}^{j}$ construites par Laumon, Rapoport et Stuhler afin de démontrer la correspondance de Langlands locale en égale caractéristique. Concrètement, on a obtenu une suite spectrale dont la partie cuspidale dégénère, conduisant au résultat de la proposition 10.17. Au vu de la proposition 10.5, qui résume les résultats de [L-R-S] que nous utilisons, on a en fait démontré :

$$
\operatorname{Hom}_{\mathrm{GL}_{d}\left(F_{o}\right)}\left(\Psi_{d}^{2(d-1)-j}(d-1)\left(\xi_{o}\right), \pi_{o}^{\vee}\right)=\left\{\begin{array}{l}
\mathrm{JL}\left(\pi_{o}\right) \otimes\left(\sigma_{d}\left(\pi_{o}\right) \otimes||^{\frac{1-d}{2}}\right) \quad \text { si } j=d-1 ; \\
0 \text { sinon. }
\end{array}\right.
$$

C'est équivalent à l'assertion de la conjecture de Drinfeld-Carayol. En effet, notant $E$ l'espace de $\Psi=\Psi_{d}^{2(d-1)-j}(d-1)\left(\xi_{o}\right)$ et $V$ l'espace de $\pi=\pi_{o}^{\vee}$,

$$
\begin{aligned}
\operatorname{Hom}_{\mathrm{GL}_{d}\left(F_{o}\right)}(\pi, \Psi) \times \operatorname{Hom}_{\mathrm{GL}_{d}\left(F_{o}\right)}(\Psi, \pi) & \longrightarrow \overline{\mathbb{Q}}_{\ell} \\
(f \quad, \quad g) & \longmapsto g \circ f
\end{aligned}
$$

constitue une dualité parfaite : $\operatorname{Hom}_{\mathrm{GL}_{d}\left(F_{o}\right)}(\pi, \pi) \simeq \overline{\mathbb{Q}}_{\ell}$ car $\pi_{o}$ est irréductible. Soit $0 \neq g \in$ $\operatorname{Hom}_{\mathrm{GL}_{d}\left(F_{o}\right)}(\Psi, \pi)$; choisissant $\tilde{f} \neq 0$ dans $\operatorname{Hom}_{\mathrm{GL}_{d}\left(F_{o}\right)}(V, E / \operatorname{ker} g) \simeq \overline{\mathbb{Q}}_{\ell}(\operatorname{car} \operatorname{im} g=V)$, on peut relever $\tilde{f}$ en un élément $f$ de $\operatorname{Hom}_{\mathrm{GL}_{d}\left(F_{o}\right)}(\pi, \Psi)$, parce qu'une représentation cuspidale est projective dans la catégorie des représentations lisses de $\mathrm{GL}_{d}\left(F_{o}\right)$ de caractère central fixé (sa restriction à $\mathrm{GL}_{d}^{\prime}\left(F_{o}\right)$ est une représentation finie; on utilise à nouveau le lemme 10.15 et le fait que $\mathrm{GL}_{d}\left(F_{o}\right)$ est engendré par son centre et par $\left.\mathrm{GL}_{d}^{\prime}\left(F_{o}\right)\right)$. Un tel relèvement vérifie $g \circ f \neq 0$.

Prenons maintenant $f \neq 0$ dans $\operatorname{Hom}_{\mathrm{GL}_{d}\left(F_{o}\right)}(\pi, \Psi)$ et construisons dans $\operatorname{Hom}_{\mathrm{GL}_{d}\left(F_{o}\right)}(\Psi, \pi)$ un élément $g$ tel que $g \circ f \neq 0:$ partant de $0 \neq \tilde{g} \in \operatorname{Hom}_{\mathrm{GL}_{d}\left(F_{o}\right)}(\operatorname{im} f, V) \simeq \overline{\mathbb{Q}}_{\ell}$, on utilise le fait qu'une représentation cuspidale est également injective dans la catégorie des représentations lisses de $\mathrm{GL}_{d}\left(F_{o}\right)$ de caractère central fixé (cela résulte de la remarque 10.16). Finalement, $B=\operatorname{Hom}_{\mathrm{GL}_{d}\left(F_{o}\right)}(\Psi, \pi)$ est de dimension finie (on a calculé cet espace précédemment) et, notant $A=\operatorname{Hom}_{\mathrm{GL}_{d}\left(F_{o}\right)}(\pi, \Psi)$, on dipose d'injections $A \subset B^{*}$ et $B \subset A^{*}$; ces deux espaces sont donc de même dimension, et l'on a l'égalité $A=B^{*}$. 
On en déduit :

$$
\left(\Psi_{d}^{j}\left(\xi_{o}\right)\right)(d-1)\left[\pi_{o}\right]=\left\{\begin{array}{l}
\mathrm{JL}\left(\pi_{o}\right) \otimes\left(\sigma_{d}\left(\pi_{o}\right) \otimes||^{\frac{d-1}{2}}\right) \quad \text { si } j=d-1 \\
0 \quad \text { sinon. }
\end{array}\right.
$$

Il ne reste plus qu'à « simplifier » les torsions de Tate; la conjecture de Drinfeld-Carayol est démontrée.

\section{Annexe A}

\section{Cohomologie $l$-adique des espaces analytiques, d'après Berkovich}

A.1. Cohomologie à support compact. - Dans [B1] et [B2], seulement la cohomologie étale à coefficients dans un faisceau de torsion est définie et étudiée. Ne disposant pas d'une théorie générale de la cohomologie $\ell$-adique, nous donnons ci-dessous une définition de la cohomologie $\ell$-adique à support compact, valable pour une certaine classe d'espaces analytiques qui inclut les revêtements $\Sigma_{n}^{d}$ de Drinfeld. Les résultats qui suivent proviennent de notes non publiées de Berkovich, et sont entièrement dûs à Berkovich qui a aimablement autorisé l'auteur à les reproduire ici.

Soit $K$ un corps local non-archimédien, de corps résiduel $\kappa$ de caractéristique $p$, et soit $X$ un $K$-espace analytique. On supposera toujours dans ce qui suit que $X$ est de Hausdorff. On dit que $X$ est quasi-algébrique si tout point $x \in X$ possède un voisinage de la forme $V_{1} \cup \ldots \cup V_{n}$, où chaque $V_{i}$ est un affinoïde de $X$ qui est isomorphe à un affinoïde de $S_{i}^{a n}$, pour un certain schéma $S_{i}$ de type fini sur $K$.

Remarque. - Un K-espace analytique lisse est automatiquement quasi-algébrique (cf. [B2], preuve du corollaire 5.6 : si $\varphi: Y \rightarrow X$ est un morphisme quasi-étale et $X$ est quasi-algébrique, alors $Y$ est également quasi-algébrique).

Par ailleurs, un sous-ensemble ouvert $\mathcal{U}$ de $X$ est dit distingué s'il est de la forme $V \backslash U$, où $U \subset V$ sont deux domaines analytiques compacts de $X$. Il est clair que la classe des sousensembles ouverts distingués est stable par union et intersection finies.

Finalement, fixons une clôture algébrique $\bar{K}$ de $K$ ainsi qu'une clôture séparable $K^{s} \subset \bar{K}$; pour tout $K$-espace analytique $Y$, nous noterons $Y_{\bar{K}}=Y \hat{\otimes}_{K} \widehat{\bar{K}}$. Fixons également un nombre premier $\ell \neq p$; un faisceau $\ell$-adique lisse est un système projectif $F=\left\{F_{n}\right\}_{n \geq 0}$ de faisceaux finis localement constants $F_{n}$ en $\mathbb{Z} / \ell^{n+1} \mathbb{Z}$-modules tel que $F_{n+1} \otimes \mathbb{Z} / \ell^{n} \mathbb{Z} \stackrel{\sim}{\rightarrow} F_{n}$.

Lemme A.1 (Berkovich). - Soit $\mathcal{U}$ un sous-ensemble ouvert distingué d'un $K$-espace analytique quasi-algébrique $X$ et soit $A$ un anneau commutatif noethérien de caractéristique $n$ premier à $p$. Alors pour tout A-module fini, étale et localement constant $F$, les groupes de cohomologie $\mathrm{H}_{c}^{q}\left(\mathcal{U}_{\bar{K}}, F\right), q \geq 0$, sont des A-modules finis.

Démonstration. - Supposons pour commencer que $X$ est compact et que $\mathcal{U}=X$. Alors l'assertion est prouvée dans $[\mathbf{B 2}$ 5.6, dans le cas $A=\mathbb{Z} / n \mathbb{Z}$. La même preuve fonctionne pour $A$ quelconque.

Dans le cas général, soit $\mathcal{U}=V \backslash U$, où $U \subset V$ sont deux sous-domaines analytiques compacts de $X$. Le lemme résulte alors de la suite exacte longue

$$
\ldots \rightarrow \mathrm{H}^{q-1}\left(U_{\bar{K}}, F\right) \rightarrow \mathrm{H}_{c}^{q}\left(\mathcal{U}_{\bar{K}}, F\right) \rightarrow \mathrm{H}^{q}\left(V_{\bar{K}}, F\right) \rightarrow \mathrm{H}^{q}\left(U_{\bar{K}}, F\right) \rightarrow \ldots
$$


Proposition A.2 (Berkovich). - Soit $\mathcal{U}$ un sous-ensemble ouvert distingué d'un $K$-espace analytique quasi-algébrique $X$. Alors pour tout faisceau $\ell$-adique lisse $F=\left\{F_{n}\right\}_{n \geq 0}$ sur $X$ et tout entier $q \geq 0$, les groupes de cohomologie $\left\{\mathrm{H}_{c}^{q}\left(\mathcal{U}_{\bar{K}}, F_{n}\right)\right\}_{n \geq 0}$ forment un système projectif $A R$ - $\ell$-adique de groupes de $\ell$-torsion finis.

Démonstration. - L'assertion de la proposition est un corollaire immédiat de [SGA5], Exp. V 5.3.1, appliqué au $\partial$-foncteur exact $F \mapsto \mathrm{H}_{c}^{q}\left(\mathcal{U}_{\bar{K}}, F\right)$ défini sur la catégorie des faisceaux de $\ell$-torsion finis localement constants. La condition nécessaire à l'application du résultat dans loc. cit. est vérifiée en vertu du lemme précédent.

Nous pouvons maintenant définir la cohomologie d'un $K$-espace analytique quasi-algébrique $X$ à coefficients dans un faisceau $\ell$-adique lisse $F=\left\{F_{n}\right\}_{n \geq 0}$ sur $X$. Tout d'abord, pour $\mathcal{U} \subset X$ un ouvert distingué, Berkovich pose

$$
\widetilde{\mathrm{H}}_{c}^{q}\left(\mathcal{U}_{\bar{K}}, F\right):={\underset{\leftarrow}{n}}_{\lim _{c}} \mathrm{H}_{c}^{q}\left(\mathcal{U}_{\bar{K}}, F_{n}\right) .
$$

Il résulte de la proposition A.2 que $F \mapsto \widetilde{\mathrm{H}}_{c}^{q}\left(\mathcal{U}_{\bar{K}}, F\right)$ est un $\partial$-foncteur exact de la catégorie des

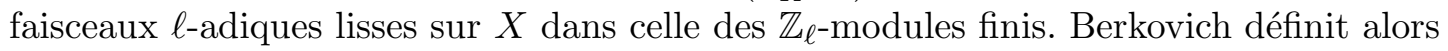

$$
\left.\mathrm{H}_{c}^{q}\left(X_{\bar{K}}, F\right):=\underset{\overrightarrow{\mathcal{U}}}{\lim _{c}} \widetilde{\mathrm{H}}_{\bar{K}}^{q}, F\right) .
$$

La cohomologie à coefficients dans $F \otimes \mathbb{Q}_{\ell}$ et $F \otimes \overline{\mathbb{Q}}_{\ell}$ se déduit en posant

$$
\widetilde{\mathrm{H}}_{c}^{q}\left(\mathcal{U}_{\bar{K}}, F \otimes \mathbb{Q}_{\ell}\right)=\widetilde{\mathrm{H}}_{c}^{q}\left(\mathcal{U}_{\bar{K}}, F\right) \otimes \mathbb{Q}_{\ell} \quad \text { et } \quad \widetilde{\mathrm{H}}_{c}^{q}\left(\mathcal{U}_{\bar{K}}, F \otimes \overline{\mathbb{Q}}_{\ell}\right)=\widetilde{\mathrm{H}}_{c}^{q}\left(\mathcal{U}_{\bar{K}}, F\right) \otimes \overline{\mathbb{Q}}_{\ell},
$$

puis en passant à la limite inductive sur $\mathcal{U}$ comme précédemment.

La situation suivante s'avère utile dans la pratique pour le calcul de la cohomologie $\ell$-adique : supposons que soit donné un recouvrement localement fini $\left\{\mathcal{U}_{\delta}\right\}_{\delta \in \Delta}$ de $X$ par des ouverts distingués. Ici, $\Delta$ désigne le nerf du recouvrement et $\mathcal{U}_{\delta}$ l'intersection de tous les ouverts correspondant aux sommets du simplexe $\delta \in \Delta$. Soit $\Delta_{i}$ l'ensemble des simplexes de $\Delta$ de dimension $i$.

Lemme A.3 (Berkovich). - On dispose d'une suite spectrale :

$$
\mathrm{E}_{1}^{i, j}=\bigoplus_{\delta \in \Delta_{-i}} \widetilde{\mathrm{H}}_{c}^{j}\left(\left(\mathcal{U}_{\delta}\right)_{\bar{K}}, F\right) \Longrightarrow \mathrm{H}_{c}^{i+j}\left(X_{\bar{K}}, F\right)
$$

Les différentielles sont induites par les inclusions $\mathcal{U}_{\delta^{\prime}} \subset \mathcal{U}_{\delta}$, pour $\delta \subset \delta^{\prime}$ deux simplexes emboîtés ; la cohomologie avec support est covariante pour l'inclusion, d'où le signe -.

Démonstration. - Soit $\Delta^{\prime}$ le sous-complexe de $\Delta$ défini par un sous-ensemble fini $\Delta_{0}^{\prime} \subset \Delta_{0}$, et soit $X^{\prime}=\bigcup_{\delta \in \Delta_{0}^{\prime}} \mathcal{U}_{\delta}$. Alors il existe pour tout entier $n \geq 1$ une suite spectrale de groupes finis abéliens

$$
\mathrm{E}_{1}^{i, j}=\bigoplus_{\delta \in \Delta_{-i}^{\prime}} \mathrm{H}_{c}^{j}\left(\left(\mathcal{U}_{\delta}\right)_{\bar{K}}, F_{n}\right) \Longrightarrow \mathrm{H}_{c}^{i+j}\left(X_{\bar{K}}^{\prime}, F_{n}\right) .
$$

Cela induit une suite spectrale similaire à coefficients dans $F$. Comme $\mathrm{H}_{c}^{q}\left(X_{\bar{K}}, F\right)=\lim _{\longrightarrow} \mathrm{H}_{c}^{q}\left(X_{\bar{K}}^{\prime}, F\right)$, nous obtenons donc la suite spectrale mentionnée.

Ainsi la cohomologie $\ell$-adique avec support peut-elle se calculer à l'aide d'un recouvrement de Čech. Une autre propriété essentielle que l'on utilise est la suivante (théorème de comparaison) :

Théorème A.4 (Berkovich). - Soit $X$ un schéma propre sur $K$ et $F$ un faisceau $\ell$-adique lisse. Il existe pour tout $j \geq 0$ un isomorphisme canonique :

$$
\mathrm{H}_{c}^{j}\left(X_{\bar{K}}, F\right) \stackrel{\sim}{\rightarrow} \mathrm{H}_{c}^{j}\left(X_{\bar{K}}^{a n}, F^{a n}\right) .
$$


Démonstration. - Le résultat étant connu dans le cas où $F=F_{n}$ est un faisceau abélien de torsion (voir [B1 7.1.1), il suffit de passer à la limite sur $n$, ce qui est tout à fait licite lorsque $X$ est propre.

Terminons par un résultat de dimension cohomologique :

Proposition A.5 (Berkovich). - Soit $X$ un $K$-espace analytique quasi-algébrique de dimension d et $F$ un faisceau $\ell$-adique lisse. Alors $\mathrm{H}_{c}^{j}\left(X_{\bar{K}}, F\right)=0$ pour tout $j>2 d$.

Démonstration. - L'assertion, pour $F=F_{n}$ un faisceau abélien de torsion, résulte de B1] Corollary 5.3.8. On passe à la limite sur $n$ puis sur $\mathcal{U}$.

A.2. Cohomologie sans support. - Soit $X$ un $K$-espace analytique quasi-algébrique, de dimension $d$, que l'on supposera toujours de Hausdorff et soit $F=\left\{F_{n}\right\}_{n \geq 0}$ un faisceau $\ell$-adique lisse. Berkovich pose d'abord, pour $V \subset X$ un sous-domaine analytique compact :

$$
\mathrm{H}^{q}\left(V_{\bar{K}}, F\right):={\underset{n}{n}}_{\lim ^{q}} \mathrm{H}^{q}\left(V_{\bar{K}}, F_{n}\right) \otimes_{\mathbb{Z}_{\ell}} \mathbb{Q}_{\ell} ;
$$

puis il définit la cohomologie $\ell$-adique sans support de $X$, sachant que les sous-domaines analytiques compacts de $X$ forment un ensemble filtré :

$$
\mathrm{H}^{q}\left(X_{\bar{K}}, F\right):={\underset{\iota}{\zeta}}_{\lim ^{q}} \mathrm{H}^{q}\left(V_{\bar{K}}, F\right) \text {. }
$$

On peut également utiliser la variante suivante : définissant, pour $\mathcal{U}$ un ouvert distingué,

$$
\widetilde{\mathrm{H}}^{q}\left(\mathcal{U}_{\bar{K}}, F\right):={\underset{n}{n}}_{\lim } \mathrm{H}^{q}\left(\mathcal{U}_{\bar{K}}, F_{n}\right) \otimes_{\mathbb{Z}_{\ell}} \mathbb{Q}_{\ell},
$$

on a l'égalité

$$
\mathrm{H}^{q}\left(X_{\bar{K}}, F\right)=\lim _{\breve{U}} \widetilde{H}^{q}\left(\mathcal{U}_{\bar{K}}, F\right) .
$$

Ceci a bien un sens car le lemme A.1 et la proposition A.2 sont également valables pour la cohomologie sans support, avec les mêmes démonstrations. Supposons maintenant que $X$ soit lisse; le théorème de dualité de Poincaré établi par Berkovich (voir [B1], thm. 7.3.1) fournit un isomorphisme canonique $\mathrm{H}^{q}\left(\mathcal{U}_{\bar{K}}, F_{n}\right) \simeq \operatorname{Hom}_{\mathbb{Q}_{l}}\left(\mathrm{H}_{c}^{2 d-q}\left(\mathcal{U}_{\bar{K}}, F_{n}\right)(d), \mathbb{Q}_{l}\right)$, où $(d)$ désigne un twist de Tate. En passant à la limite sur $n$, on obtient une dualité $: \widetilde{\mathrm{H}}^{q}\left(\mathcal{U}_{\bar{K}}, F\right) \simeq$ $\operatorname{Hom}_{\mathbb{Q}_{\ell}}\left(\widetilde{\mathrm{H}}_{c}^{2 d-q}\left(\mathcal{U}_{\bar{K}}, F\right)(d), \mathbb{Q}_{\ell}\right)$; faisant croître $\mathcal{U}$, on démontre ainsi :

Théorème A.6 (Berkovich). - Soit X un K-espace analytique lisse de dimension d. Il existe une dualité de Poincaré :

$$
\mathrm{H}^{q}\left(X_{\bar{K}}, F\right) \simeq \operatorname{Hom}_{\mathbb{Q}_{\ell}}\left(\mathrm{H}_{c}^{2 d-q}\left(X_{\bar{K}}, F\right)(d), \mathbb{Q}_{\ell}\right) .
$$

Autrement dit, la cohomologie $\ell$-adique sans support est le dual algébrique de la cohomologie avec support $\mathrm{H}_{c}^{2 d-}$, modulo un twist de Tate.

Le lemme suivant affirme qu'il est possible de calculer la cohomologie $\ell$-adique sans support à l'aide d'un recouvrement de Čech, du moins sous certaines hypothèses.

Lemme A.7. - Soit $\left\{\mathcal{U}_{\delta}\right\}_{\delta \in \Delta}$ un recouvrement fini d'un $K$-espace quasi-algébrique compact $X$ par des ouverts distingués. On dispose d'une suite spectrale:

$$
\mathrm{E}_{1}^{i, j}=\bigoplus_{\delta \in \Delta_{i}} \widetilde{\mathrm{H}}^{j}\left(\left(\mathcal{U}_{\delta}\right)_{\bar{K}}, \mathbb{Q}_{\ell}\right) \Longrightarrow \mathrm{H}^{i+j}\left(X_{\bar{K}}, \mathbb{Q}_{\ell}\right) .
$$


Démonstration. - On dispose de la suite spectrale de Cartan-Leray, pour tout $n \geq 0$ :

$$
\mathrm{E}_{1}^{i, j}=\bigoplus_{\delta \in \Delta_{i}} \mathrm{H}^{j}\left(\left(\mathcal{U}_{\delta}\right)_{\bar{K}}, \mathbb{Z} / \ell^{n} \mathbb{Z}\right) \Longrightarrow \mathrm{H}^{i+j}\left(X_{\bar{K}}, \mathbb{Z} / \ell^{n} \mathbb{Z}\right)
$$

Cette suite spectrale passe à la limite projective sur $n$, compte tenu des définitions et remarques en début de paragraphe.

A.3. Espaces analytiques et actions de groupes. - Un des intérêts de la théorie des espaces analytiques de Berkovich est qu'elle inclut un traitement des espaces avec opérateurs. Ces résultats étant non publiés à ce jour (voir cependant [B3]), nous incluons ci-dessous un résumé des notions que nous utiliserons.

A.3.1. $G$-espaces. - Soit $X$ un $K$-espace analytique et $\mathcal{G}(X)$ le groupe des automorphismes de $X$. Berkovich munit $\mathcal{G}(X)$ d'une certaine topologie (voir B2] $\S 6$ ); un groupe topologique $G$ agira donc continûment sur $X$ si et seulement si l'homomorphisme induit $G \rightarrow \mathcal{G}(X)$ est continu.

Définition A.8. - Un espace analytique muni d'une action continue d'un groupe topologique $G$ est appelé un $G$-espace.

Exemple. - 1. L'action de $\mathrm{Gal}\left(K^{s} / K\right)$ sur $X_{\bar{K}}$ est continue.

2. Si $G$ agit continûment sur un schéma formel $\mathfrak{X}$ localement de présentation finie sur $K$, alors l'action de $G$ induite sur la fibre générique (au sens de Raynaud) $\mathfrak{X}_{\eta}$ de $\mathfrak{X}$ est continue. En particulier, $\mathrm{GL}_{d}(K)$ agit continûment sur le demi-plan supérieur généralisé de Drinfeld $\Omega_{K}^{d}$ défini sur un corps local $K$ (donc également sur les revêtements $\Sigma_{n}^{d}$ de $\Omega^{d} \hat{\otimes}_{K} \widehat{K}^{n r}$, par construction même de ces derniers).

Les couples $X(G)$ (en notation de Berkovich), où $G$ est un groupe topologique et $X$ un $G$ espace, sont les objets de la catégorie des espaces analytiques avec opérateurs. Un morphisme $\varphi: X^{\prime}\left(G^{\prime}\right) \rightarrow X(G)$ consiste en un homomorphisme continu de groupes topologiques $\nu_{\varphi}: G^{\prime} \rightarrow$ $G$ et un morphisme d'espaces analytiques $\varphi: X^{\prime} \rightarrow X$ compatible avec l'homomorphisme $\nu_{\varphi}$. Un morphisme $U(G) \rightarrow X(G)$ est par définition quasi-étale (resp. étale) si le morphisme sousjacent $U \rightarrow X$ est quasi-étale (resp. étale). On renvoie le lecteur à [B1] §4 (resp. [B2] §3) pour les définitions relatives à la topologie étale (resp. quasi-étale) sur les espaces analytiques. On note $X(G)_{e ́ t}$ le site étale et $X(G)_{e ́ t}^{\sim}$ le topos correspondant.

A.3.2. $G$-faisceaux. - Lorsque $G$ est muni de la topologie discrète (on écrira $G^{d}$ par la suite), on définit un $G$-faisceau étale sur $X$ comme suit : c'est un faisceau étale $F$ sur $X$ muni d'une action de $G$ sur $F$ compatible avec l'action de $G$ sur $X$ (concrètement, pour tout morphisme étale $U \rightarrow X$ et tout $g \in G$, il existe une bijection fonctorielle $F(U) \stackrel{\sim}{\rightarrow} F\left({ }^{g} U\right): f \mapsto{ }^{g} f$, où ${ }^{g} U=U \times \times_{X, g^{-1}} X$, telle que $\left.{ }^{g h} f={ }^{g}\left({ }^{h} f\right)\right)$. Si $F$ est un tel $G$-faisceau, alors pour tout morphisme étale $U(H) \rightarrow X(G)$, où $H$ est un sous-groupe de $G$, l'ensemble $F(U)$ est muni d'une action canonique de $H$ : pour $h \in H$, le morphisme $h^{-1}: U \rightarrow U$ induit un isomorphisme $U \stackrel{\sim}{\rightarrow}{ }^{h} U$ au-dessus de $X$; ce dernier induit à son tour une bijection $\sigma(h): F\left({ }^{h} U\right) \stackrel{\sim}{\rightarrow} F(U)$, et l'action de $H$ sur $F(U)$ est alors définie par $h f=\sigma(h)\left({ }^{h} f\right)$.

Pour passer au cas d'un groupe topologique $G$ quelconque, rappelons tout d'abord qu'en vertu du lemme clef 7.2 de [B2], pour un morphisme quasi-étale $U \rightarrow X$ fixé avec $U$ compact, l'action de $G$ sur $X$ se prolonge de manière canonique en une action continue sur $U$ d'un sous-groupe ouvert convenable $G_{U} \subset G$. On remarque que, si $F$ est un $G^{d}$-faisceau étale, alors pour tout 
morphisme quasi-étale $U \rightarrow X$ avec $U$ compact, il existe une action canonique de $G_{U}$ sur $\left.F\right|_{U}$ compatible avec l'action de $G \operatorname{sur} X$. En particulier, le groupe $G_{U}$ agit sur $F(U)$.

Définition A.9. - Un $G$-faisceau étale sur un $G$-espace $X$ est un $G^{d}$-faisceau étale $F$ tel que pour tout morphisme quasi-étale $U \rightarrow X$ avec $U$ compact l'action de $G_{U}$ sur $F(U)$ est discrète (i.e. le stabilisateur de toute section $f \in F(U)$ est un sous-groupe ouvert de $G_{U}$ ).

Remarque A.10. - Il résulte directement de cette définition que si $F$ est un $G$-faisceau sur $X$, alors $\Gamma_{c}(X, F)$ est un $G$-module lisse au sens de la théorie des représentations.

Soit $X$ un $G$-espace. Considérons le morphisme $b: X(\{1\}) \rightarrow X(G)$; Berkovich montre que $b^{*} F$ est un $G$-faisceau étale pour tout $F \in X(G) \tilde{e} t$. Mieux encore :

Théorème A.11 (Berkovich). — Soit X un G-espace; le morphisme b* induit une équivalence de catégories entre le topos $X(G) \tilde{e}$ et la catégorie des $G$-faisceaux étales sur $X$.

Pour finir, soit $\Lambda$ un anneau, que l'on regarde comme faisceau constant sur $X$ muni de l'action canonique de $G$ sur $\Lambda_{X}$ (c'est donc un $G$-faisceau) ; nous appelons $\Lambda-G$-module étale sur $X$ un faisceau $F$ sur $X$ qui est à la fois un $\Lambda$-module et un $G$-faisceau étale.

A.4. Résolution flasque de Godement et application. - Soit $X$ un $G$-espace et $\Lambda$ un anneau. Berkovich applique la construction de la résolution flasque de Godement (voir [SGA4] Exp. XVII §4.2) au site étale $X(G)_{e ́ t}$ afin de construire, pour $F$ un faisceau étale abélien sur $X(G)$, une résolution à droite $\mathcal{C}(F)$ de $F$ vérifiant (cf. loc. cit. $\S 4.2 .3$ ) :

$-\mathcal{C}^{m}(F)$ est un faisceau flasque;

- le foncteur $F \mapsto \mathcal{C}^{m}(F)$ est exact;

- la fibre du complexe $\mathcal{C} \cdot(F)$ en $x \in X$ est une résolution canoniquement scindée de $F_{x}$.

Cette construction est fonctorielle. De plus, elle conserve les structures supplémentaires (cf. remarque 4.2.5 dans loc. cit.). Par conséquent, si $b^{*} F$ est un $\Lambda-G$-module étale, alors il en est de même des transformés $b^{*} \mathcal{C}^{m}(F)$.

Poursuivant, Berkovich montre que le faisceau $b^{*} C^{m}(F)$ est mou pour tout $m \geq 0$ (au sens de B2 §3). Ainsi, en vertu du lemme 3.2 de [B2], si l'on suppose $X$ paracompact, alors le complexe $b^{*} \mathcal{C}(F)$ est $R \Gamma$-acyclique; à fortiori $: \mathrm{H}_{c}^{j}\left(X, b^{*} \mathcal{C}^{m}(F)\right)=0$ pout tout entier $j>0$.

Dans [H1], Harris esquisse comment utiliser la construction précédente afin de démontrer la lissité de l'action sur les groupes de cohomologie avec support en construisant notamment un complexe lisse qui calcule cette cohomologie. Nous énonçons ici le résultat dans sa généralité et détaillons la construction (voir également la version plus « sophistiquée » décrite dans [H2] $\$ 2$ ).

Proposition A.12 (Berkovich-Harris). - Soit X un G-espace quasi-algébrique sur K. On suppose que $G$ contient un sous-groupe ouvert qui est un pro-p-groupe, avec $p \neq \ell$. Alors, pour tout ouvert distingué $\mathcal{U} \subset X$, l'action du stabilisateur $G_{\mathcal{U}}$ de $\mathcal{U}$ sur $\widetilde{\mathrm{H}}_{c}^{j}\left(\mathcal{U}_{\bar{K}}, \mathbb{Q}_{\ell}\right), j \geq 0$, est lisse (ceci est dî̀ à Berkovich). On peut construire un complexe $C \cdot(\mathcal{U})$ de $\mathbb{Q}_{\ell}-G_{\mathcal{U}}$-modules lisses (i.e. de $\mathbb{Q}_{\ell}$-représentations lisses de $\left.G_{\mathcal{U}}\right)$ qui calcule la cohomologie avec support:

$$
\widetilde{\mathrm{H}}_{c}^{j}\left(\mathcal{U}_{\bar{K}}, \mathbb{Q}_{\ell}\right) \stackrel{\sim}{\rightarrow} h^{j}(C \cdot(\mathcal{U})) \quad \forall j \geq 0
$$

(isomorphismes $G_{\mathcal{U}}$-équivariants). Cette construction est fonctorielle en $\mathcal{U}:$ pour $\mathcal{U}^{\prime} \subset \mathcal{U}$ deux ouverts distingués emboîtés, il existe une application canonique $C^{\cdot}(\mathcal{U}) \rightarrow C^{\cdot}\left(\mathcal{U}^{\prime}\right)$ compatible aux isomorphismes (11) et aux actions (i.e. cette application est $G_{\mathcal{U}} \cap G_{\mathcal{U}^{\prime}}$-équivariante). 
Remarque. - Ecrivant $\mathcal{U}=U \backslash V$, où $U$ et $V$ sont deux compacts analytiques et appliquant le lemme clef 7.2 de [B2] (cf. paragraphe A.3.2), on voit que $\mathcal{U}$ est invariant sous l'action d'un sous-groupe ouvert de $G$. Le stabilisateur $G_{\mathcal{U}}$ est donc ouvert.

Démonstration. - Étant donnée la définition de la cohomologie $\ell$-adique à support (valable pour un espace analytique quasi-algébrique; voir section A.11), nous construisons d'abord, pour tout $n \geq 1$, une résolution $R \Gamma_{c^{-}}$acyclique du $\mathbb{Z} / \ell^{n} \mathbb{Z}-G_{\mathcal{U}}$-module constant $\mathbb{Z} / \ell^{n} \mathbb{Z}$ sur $\mathcal{U}_{\bar{K}}$ par des $\mathbb{Z} / \ell^{n} \mathbb{Z}-G_{\mathcal{U}}$-modules étales. Afin de donner un sens au passage à la limite sur $n$, nous suivons Harris ([H2] §2) et introduisons la notion suivante : un système $\ell$-divisible $T$ de $G_{\mathcal{U}}$-modules sur $\mathcal{U}_{\bar{K}}$ (resp. de $G$-modules sur $X_{\bar{K}}$ ) est une famille $\left\{T_{n}\right\}$ de $\mathbb{Z} / \ell^{n} \mathbb{Z}-G_{\mathcal{U}}$-modules étales sur $\mathcal{U}_{\bar{K}}$ (resp. de $\mathbb{Z} / \ell^{n} \mathbb{Z}-G$-modules étales sur $X_{\bar{K}}$ ), munie de morphismes $i=i_{n^{\prime}, n}: T_{n^{\prime}} \rightarrow T_{n}$ et $j=j_{n, n-n^{\prime}}: T_{n} \rightarrow T_{n-n^{\prime}}$ pour tout $n>n^{\prime} \geq 1$ tels que $T_{n}$ est libre sur $\mathbb{Z} / \ell^{n} \mathbb{Z}, i$ identifie $T_{n^{\prime}}$ avec la $\ell^{n^{\prime}}$-torsion dans $T_{n}$ et la suite $0 \rightarrow T_{n^{\prime}} \stackrel{i}{\rightarrow} T_{n} \stackrel{j}{\rightarrow} T_{n-n^{\prime}} \rightarrow 0$ est exacte.

Afin que la construction soit canonique, lorsque $\mathcal{U}$ varie, on écrira plutôt une résolution du faisceau constant $\mathbb{Z} / \ell^{n} \mathbb{Z}$ par des $G$-modules sur l'espace total $X_{\bar{K}}$; on considérera ensuite les restrictions à un ouvert $\mathcal{U}_{\bar{K}}$. La construction est basée sur la remarque suivante : si $T=\left\{T_{n}\right\}$ est un système $\ell$-divisible de $G$-modules sur $X_{\bar{K}}$, alors il en est de même de $b^{*} \mathcal{C}^{m} b^{*-1}(T)=\left\{b^{*} \mathcal{C}^{m} b^{*-1}\left(T_{n}\right)\right\}$, relativement aux morphismes $b^{*} \mathcal{C}^{m} b^{*-1}(i)$ et $b^{*} \mathcal{C}^{m} b^{*-1}(j)$. Ceci résulte de l'exactitude du foncteur $b^{*}$ et des propriétés du foncteur $\mathcal{C}^{m}$ énoncées plus haut. On obtient donc :

Lemme A.13. - Pour tout $n$, le faisceau constant $\mathbb{Z} / \ell^{n} \mathbb{Z}$ admet une résolution $R \Gamma_{c}$-acyclique :

$$
0 \rightarrow \mathbb{Z} / \ell^{n} \mathbb{Z} \rightarrow T_{n}^{0} \rightarrow T_{n}^{1} \cdots \rightarrow T_{n}^{m} \rightarrow \cdots
$$

telle que $\left\{T_{n}^{m}\right\}$ est pour tout $m$ un système $\ell$-divisible de $G$-modules sur $X_{\bar{K}}$.

Mieux encore, il est possible de construire une résolution finie. En effet, si $d$ désigne la dimension de $X$, on a déjà vu (voir proposition A.5) que $\mathrm{H}_{c}^{j}(U, F)=0$ pour tout $j>2 d$, tout faisceau de torsion localement constant d'ordres premiers à la caractéristique résiduelle $p$ et tout morphisme étale $U \rightarrow X_{\bar{K}}$. On utilise alors le foncteur de tronquature $\tau^{\leq m}$ sur les complexes, défini par :

$$
\tau^{\leq m}\left(C^{\cdot}\right)^{i}=C^{i}, i<m ; \quad \tau^{\leq m}\left(C^{\cdot}\right)^{m}=\operatorname{ker}\left(C^{m} \rightarrow C^{m+1}\right) ; \quad \tau^{\leq m}\left(C^{\cdot}\right)^{i}=0, i>m .
$$

Il résulte de la remarque précédente que les inclusions $\tau^{\leq m}\left(T_{n}^{\cdot}\right) \rightarrow T_{n}^{\cdot}$ sont des quasi-isomorphismes pour tout $n$ et tout $m \geq 2 d$. Nous posons alors $T_{n}^{\prime \cdot}=\tau^{\leq 2 d+1}\left(T_{n}^{\cdot}\right)$; on obtient (en oubliant les ' pour alléger les notations) :

Lemme A.14. - Pour tout $n$, le faisceau constant $\mathbb{Z} / \ell^{n} \mathbb{Z}$ admet une résolution $R \Gamma_{c^{-}}$-acyclique de longueur finie :

$$
0 \rightarrow \mathbb{Z} / \ell^{n} \mathbb{Z} \rightarrow T_{n}^{0} \rightarrow T_{n}^{1} \cdots \rightarrow T_{n}^{m} \rightarrow \cdots \rightarrow T_{n}^{2 d+1} \rightarrow 0
$$

telle que $\left\{T_{n}^{m}\right\}$ est pour tout $m$ un système $\ell$-divisible de $G$-modules sur $X_{\bar{K}}$.

En effet, le seul point à vérifier est l'injectivité du morphisme $i: T_{n^{\prime}}^{2 d+1} \rightarrow T_{n}^{2 d+1}$ pour tout $n>n^{\prime} \geq 1$. On s'en convainc en écrivant les diagrammes.

Calculons maintenant les groupes de cohomologie $\mathrm{H}_{c}^{m}\left(\mathcal{U}_{\bar{K}}, \mathbb{Q}_{\ell}\right)$ : d'après le lemme précédent, nous disposons d'un isomorphisme $G_{\mathcal{U}}$-équivariant $\mathrm{H}_{c}^{m}\left(\mathcal{U}_{\bar{K}}, \mathbb{Z} / \ell^{n} \mathbb{Z}\right) \simeq h^{m}\left(\Gamma_{c}\left(\mathcal{U}_{\bar{K}}, T_{n}^{\cdot}\right)\right)$ pour tout entier $m \geq 0$. Le groupe de cohomologie $\mathrm{H}_{c}^{m}\left(\mathcal{U}_{\bar{K}}, \mathbb{Z} / \ell \mathbb{Z}\right)$, en particulier, est donc un $G_{\mathcal{U}}$-module lisse ( $m \geq 0$ quelconque), en vertu de la remarque A.10, Par ailleurs, c'est un $\mathbb{Z} / \ell \mathbb{Z}$ module fini (cf. lemme A.1). Il en résulte l'existence d'un sous-groupe ouvert $H \subset G_{\mathcal{U}}$ agissant 
trivialement sur $\mathrm{H}_{c}^{m}\left(\mathcal{U}_{\bar{K}}, \mathbb{Z} / \ell \mathbb{Z}\right)$. Etant donnée l'hypothèse sur $G$, on peut même supposer que $H$ est un pro- $p$-sous-groupe normal ouvert de $G_{\mathcal{U}}$ (avec $p \neq \ell$ ). Par conséquent, $H$ agit trivialement sur tous les groupes $\mathrm{H}_{c}^{m}\left(\mathcal{U}_{\bar{K}}, \mathbb{Z} / \ell^{n} \mathbb{Z}\right), n \geq 1$ : cela résulte du lemme suivant, dont la démonstration est laissée au lecteur.

Lemme A.15. - Soit $0 \rightarrow A \rightarrow B \rightarrow C \rightarrow 0$ une suite exacte de l-groupes abéliens finis et $\varphi \in A$ ut $(B)$ tel que $\left.\varphi\right|_{A}=\operatorname{Id}_{A}$ et $\left.\varphi\right|_{C}=\operatorname{Id}_{C}$. Alors il existe $n \geq 0$ tel que $\varphi^{\ell^{n}}=\mathrm{Id}$.

Utilisant ce lemme, on raisonne par récurrence sur $n$ : la suite exacte $0 \rightarrow \mathbb{Z} / \ell^{n} \mathbb{Z} \rightarrow \mathbb{Z} / \ell^{n+1} \mathbb{Z} \rightarrow$ $\mathbb{Z} / \ell \mathbb{Z} \rightarrow 0$ induit une suite exacte $0 \rightarrow A \rightarrow \mathrm{H}_{c}^{m}\left(\mathcal{U}_{\bar{K}}, \mathbb{Z} / \ell^{n+1} \mathbb{Z}\right) \rightarrow C \rightarrow 0$, où $A$ et $C$ sont des sous-groupes de $\mathrm{H}_{c}^{m}\left(\mathcal{U}_{\bar{K}}, \mathbb{Z} / \ell^{n} \mathbb{Z}\right)$ et $\mathrm{H}_{c}^{m}\left(\mathcal{U}_{\bar{K}}, \mathbb{Z} / \ell \mathbb{Z}\right)$ respectivement. Le résultat découle alors du fait que le pro-p-groupe $H$ est uniquement $\ell$-divisible, donc toute $\ell$-torsion est triviale.

A ce stade, la lissité de l'action de $G_{\mathcal{U}}$ sur les groupes de cohomologie $\ell$-adique avec support $\mathrm{H}_{c}^{m}\left(\mathcal{U}_{\bar{K}}, \mathbb{Q}_{\ell}\right)$ est acquise : l'ouvert $H$, qui agit trivialement sur tous les groupes $\mathrm{H}_{c}^{m}\left(\mathcal{U}_{\bar{K}}, \mathbb{Z} / \ell^{n} \mathbb{Z}\right)$, agit trivialement sur la limite projective.

Remarque. - En fait, il n'est pas nécessaire d'utiliser les résolutions $R \Gamma_{c}$-acycliques $T_{n}$ afin d'établir la lissité de l'action: celle-ci résulte immédiatement de [B2] Corollary 7.8 (en utilisant le lemme précédent). Mais la construction du complexe $C^{\cdot}(\mathcal{U})$, qui est le coeur de la proposition A.12 (ce complexe sert dans la section 10.2), est fondée sur ces résolutions.

Construisons maintenant le complexe $C^{\cdot}(\mathcal{U})$ : on part du système $\ell$-divisible $\left\{\Gamma_{c}\left(\mathcal{U}_{\bar{K}}, T_{n}^{\cdot}\right)\right\}$ de complexes de $\mathbb{Z} / \ell^{n} \mathbb{Z}-G_{\mathcal{U}}$-modules (vérification immédiate, sachant que les complexes $T_{n}^{\text {. }}$

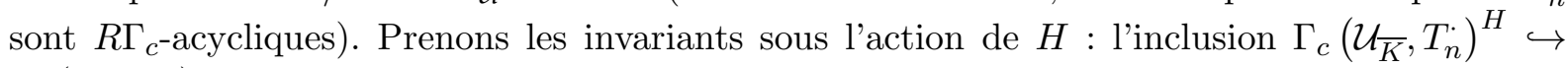
$\Gamma_{c}\left(\mathcal{U}_{\bar{K}}, T_{n}^{\cdot}\right)$ est un quasi-isomorphisme de complexes. En effet, puisque $H$ est un pro-p-groupe, alors $\mathrm{H}^{j}(H, M)=0$ pour tout module $M$ qui est uniquement de $\ell$-torsion et tout entier $j \geq 1$ (la multiplication par $p$ dans $M$ est bijective car $p$ est premier à $\ell$; pour $K$ un $p$-groupe, $\mathrm{H}^{j}(K, M)$ est de $p$-torsion pour tout $j>0$, donc est nul. C'est encore vrai pour un pro-p-groupe par passage à la limite projective). Le foncteur $\mathrm{H}^{0}(H, \bullet)$ est donc exact sur les modules $\Gamma_{c}\left(\mathcal{U}_{\bar{K}}, T_{n}^{m}\right)$, d'où il résulte un isomorphisme $h^{q}\left(\Gamma_{c}\left(\mathcal{U}_{\bar{K}}, T_{n}^{\cdot}\right)^{H}\right) \simeq h^{q}\left(\Gamma_{c}\left(\mathcal{U}_{\bar{K}}, T_{n}^{\cdot}\right)\right)^{H}=h^{q}\left(\Gamma_{c}\left(\mathcal{U}_{\bar{K}}, T_{n}^{\cdot}\right)\right)$.

Notons $C_{n}^{\cdot, H}=\Gamma_{c}\left(\mathcal{U}_{\bar{K}}, T_{n}^{\cdot}\right)^{H}$; on vérifie facilement que $C_{n}^{\cdot, H}$ est un système $\ell$-divisible de complexes de longueur finie $(=2 \mathrm{~d}+1)$ de $\mathbb{Z} / \ell^{n} \mathbb{Z}-G_{\mathcal{U}} / H$-modules (toujours d'après l'exactitude $\mathrm{du}$ foncteur $\mathrm{H}^{0}(H, \bullet)$ sur les modules considérés). Les applications $j: C_{n}^{\cdot, H} \rightarrow C_{n^{\prime}}^{,, H}$, pour $n \geq n^{\prime}$, étant surjectives, on voit que ce système $\ell$-divisible satisfait une condition de Mittag-Leffler (cf. EGA3 Chap. 0, Proposition 13.2.3), qui autorise à passer à la limite sur $n: \lim _{n} h^{m}\left(C_{n}^{\cdot, H}\right) \simeq$ $h^{m}\left(C^{\cdot, H}\right)$, où l'on note $C^{\cdot, H}=\lim _{\longleftarrow} C_{n}^{\cdot, H}$. Le complexe $C^{\cdot, H}(\mathcal{U})=C^{\cdot, H} \otimes_{\mathbb{Z}_{l}} \mathbb{Q}_{l}$ est donc un complexe de $\mathbb{Q}_{\ell}-G_{\mathcal{U}} / H$-modules lisses tel que : $h^{m}\left(C^{\cdot}, H(\mathcal{U})\right) \stackrel{\sim}{\rightarrow} \widetilde{\mathrm{H}}_{c}^{m}\left(\mathcal{U}_{\bar{K}}, \mathbb{Q}_{\ell}\right)$ (isomorphisme $G_{\mathcal{U}} / H$-équivariant, pour tout entier $m \geq 0$ ).

Si l'on remplace $H$ par un autre sous-groupe $H^{\prime} \subset H$ normal ouvert de $G_{\mathcal{U}}$, on obtient un isomorphisme naturel $C^{\cdot, H}(\mathcal{U})=\left[C^{\cdot}, H^{\prime}(\mathcal{U})\right]^{H}$ (toujours d'après l'exactitude du foncteur $\mathrm{H}^{0}(H, \bullet)$ ). Définissons alors $C^{\cdot}(\mathcal{U})=\lim _{H^{\prime} \subset H} C^{\cdot}, H^{\prime}(\mathcal{U})$. La lissité de ce complexe provient de ce qu'une limite inductive de modules lisses est lisse, et l'on dispose d'un isomorphisme $G_{\mathcal{U}}$-équivariant : $h^{m}(C \cdot(\mathcal{U})) \stackrel{\sim}{\rightarrow} \widetilde{\mathrm{H}}_{c}^{m}\left(\mathcal{U}_{\bar{K}}, \mathbb{Q}_{\ell}\right)$. Le complexe $C^{\cdot}(\mathcal{U})$ remplit donc le cahier des charges.

Remarque A.16. - Travaillant dans la catégorie dérivée de celle des $\mathbb{Q}_{\ell}-G_{\mathcal{U}}$-modules, on dispose d'un quasi-isomorphisme de complexes :

$$
C^{\cdot}(\mathcal{U}) \longrightarrow \underset{n}{\lim _{n}} \Gamma_{c}\left(\mathcal{U}_{\bar{K}}, T_{n}^{\cdot}\right) \otimes_{\mathbb{Z}_{\ell}} \mathbb{Q}_{\ell}=: D^{\cdot}(\mathcal{U})
$$


Ce morphisme est canonique, lorsque $\mathcal{U}$ varie : le diagramme suivant est commutatif

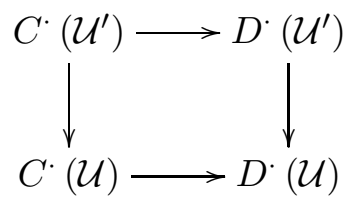

pour $\mathcal{U}^{\prime} \subset \mathcal{U}$ deux ouverts distingués emboîtés.

\section{Références}

[Ba1] I. BADULESCU, "Correspondance entre $\mathrm{GL}_{n}$ et ses formes intérieures en caractéristique positive", thèse, Université de paris XI Orsay (janvier 1999).

[Ba2] I. BADulescu, "Orthogonalité des caractères pour $\mathrm{GL}_{n}$ sur un corps local de caractéristique non nulle", Manuscripta math. 101, 49-70 (2000).

[B1] V.G. Berkovich, "Etale cohomology for non-archimedean analytic spaces", Publ. Math. I.H.E.S., 78 (1993), 5-161.

[B2] V.G. Berkovich, "Vanishing cycles for formal schemes", Invent. Math., 115 (1994), 539-571.

[B3] V.G. Berkovich, "Etale equivariant sheaves on p-adic analytic spaces", Weizmann Inst. of Science (Israel), preprint (1995).

[B-Z] J. Bernstein et A.V. Zelevinski, "Representations of the group $\mathrm{GL}(n, F)$, where $F$ is a non-archimedean local field ", Russ. Math. Surv., 31 (1976), 1-68.

[Bo-Ca] J.-F. Boutot et H. CARAyol, "Uniformisation p-adique des courbes de Shimura : les théorèmes de Čerednik et de Drinfeld", Astérisque n 196-197 (1991), 45-149.

[Boy] P. Boyer, "Mauvaise réduction des variétés de Drinfeld et conjecture de Langlands locale", Inv. Math. 138 (1999), nº 3, 573-629.

[Ca1] H. Carayol, "Variétés de Drinfeld Compactes, d'après Laumon, Rapoport et Stuhler", Séminaire BOURBAKI, 44ème année (1991-92), nº 756.

[Ca2] H. Carayol, "Non-Abelian Lubin-Tate Theory", dans : L. Clozel et J. Milne (ed), "Automorphic forms, Shimura varieties and L-functions", vol. II, Persp. in Math. 11, 15-39, Acad. Press Boston (1990).

[Ca3] H. CARAYOL, "Preuve de la conjecture de Langlands locale pour $\mathrm{GL}_{n}$ : travaux de Harris-Taylor et Henniart", Séminaire Bourbaki exp. 857 (1998-99).

[ั̆] I.V. ČEREDNIK, "Uniformization of Algebraic Curves by Discrete Arithmetic Subgroups of $\mathrm{PGL}_{2}\left(k_{w}\right)$ with compact quotients, Math. U.S.S.R. Sbornik 29 (1976) nº 1, 55-78.

[De] P. Deligne, "Lettre à Piatetskii-Shapiro", (1973).

[De-Hu] P. Deligne et D. Husemoller, "Survey of Drinfeld Modules", dans "Current Trends in Arithmetical Algebraic Geometry", Contemporary Mathematics 67, A.M.S, 1987, 25-91.

[D-K-V] P. Deligne, D.Kazhdan et M.-F. VignéRas, "Représentations des algèbres simples p-adiques ", dans I.-N. Bernstein, P. Deligne, D.Kazhdan et M.-F. Vignéras“" Représentations des groupes réductifs sur un corps local", Hermann (1984).

[Dr1] V.G. Drinfeld, "Elliptic Modules", Math. USSR Sbornik 23 (1974), 561-592.

[Dr2] V.G. Drinfeld, "Elliptic Modules II", Math. USSR Sbornik 31 (1977), 159-170.

[Dr3] V.G. Drinfeld, "Commutative Subrings of certain Noncommutative Rings", Fonct. Anal. Appl. 11 (1977), 9-12.

[Dr4] V.G. Drinfeld, "Coverings of p-adic symmetric domains", Funct. Anal. and Appl. 10 (1976), 107-115.

[Dr5] V.G. Drinfeld, "Letter to H. Carayol" (January 12th, 1980).

[Dr6] V.G. Drinfeld, "Varieties of Modules of F-sheaves", Funct. Anal. and Appl. 21 (1987), 107122.

[Dr7] V.G. DRINFELD, "Cohomology of compactified manifolds of modules of F-sheaves of rank 2", Journal of Soviet math. 46, no 1 (1989), 1789-1821. 
[EGA3] A. Grothendieck, "Eléments de Géométrie Algébrique, III : Etude cohomologique des faisceaux cohérents", Publ. Math. IHES no 11 (1961).

[Fa1] G. Faltings, "A relation between two moduli spaces studied by V.G. Drinfeld", prépublication (2001).

[Fa2] G. FALtings, "Groupschemes with strict $\mathcal{O}$-action", prépublication (2002).

[Far] L. Fargues, "Correspondances de Langlands locales dans la cohomologie des espaces de Rapoport-Zink", thèse Univ. Paris 7 (2001).

[G-M] S.I. Gelfand et Yu.I. Manin, "Homological Algebra", Springer Verlag, Berlin (1999).

[Ge] A. Genestier, "Espaces symétriques de Drinfeld", Astérisque nº 234 (1996).

[Go] R. Godement, "Topologie Algébrique et Théorie des Faisceaux", Hermann (1998).

[H1] M. HARRIS, "Supercuspidal representations in the cohomology of Drinfel'd upper half spaces; elaboration of Carayol's program", Invent. Math. 129 (1997), 75-119.

[H2] M. HARRIs, "Galois properties of cohomological automorphic forms on GL $(n)$ ", J. Math. Kyoto Univ., vol. $39 \mathrm{n}^{\circ} 2$ (1999), 299-318.

[H-T] M. HARRIS ET R. TAYLOR, "The geometry and cohomology of some simple Shimura varieties ", Ann. of Math. Studies 151, PUP (2001).

[He1] G. Henniart, "On the local Langlands conjecture for $\operatorname{GL}(n)$ : the cyclic case", Anal. Math. 123, 145-203 (1986).

[He2] G. Henniart, "Une preuve simple des conjectures de Langlands pour GL $(n)$ sur un corps padique", Inv. Math. 139 (2000), nº 2, 439-455.

[He3] G. Henniart, "La correspondance de Langlands locale : caractérisation et propriétés fonctorielles", en préparation.

[I] L. Illusie, "Complexe cotangent et déformations. I, II", Lect. Notes in Math. 239,283, Berlin Heidelberg New York, Springer $(1971,1973)$.

[L] L. Lafforgue, "Chtoucas de Drinfeld et conjecture de Ramanujan-Petersson", Astérisque $\mathrm{n}^{\circ} 243, \operatorname{SMF}(1997)$.

[Lau] G. Laumon, "Cohomology of Drinfeld Modular Varieties, part I", Cambridge University Press (1996).

[L-R-S] G. Laumon, M. Rapoport, U. Stuhler, "D-elliptic sheaves and the Langlands correspondence", Inv. Math. 113, 217-338 (1993).

[Le] B. Lemaire, "Intégrabilité locale des caractères-distributions de $\mathrm{GL}_{n}(F)$, où $F$ est un corps local non archimédien de caractéristique quelconque", Compos. Math. 100 (1996), 41-75.

[Me] W. Messing, "Crystals associated to Barsotti-Tate groups", Lect. Notes in Math. 264, Berlin, Springer (1972).

[M] D. Mumford, "An analytic construction of Degenerating curves over Complete Local Rings", Compositio Math. 24 (1972), Fasc. 3, 239-272.

[Mu] G.A. Mustafin, "Nonarchimedean Uniformization", Math. USSR Sbornik 34 (1978), 187-214.

[R-Z] M. RApoport, Th. ZINK, "Period spaces for p-divisible groups", Annals of Math. Studies 141 (1996).

[Ra1] M. RAYNAud, "Construction Analytique de Courbes en Géométrie non Archimédienne (d'après David Mumford)", Séminaire Bourbaki no 427 (1972-73), Lect. Notes in Math. 383, SpringerVerlag (1974), 171-185.

[Ra2] M. Raynaud, "Géomérie Analytique Rigide d'après Tate, Kiehl, ...", Table Ronde Anal. non Archim., Bull. Soc. Math. Fr., Mém. 39-40 (1974), 319-327.

[Ro] J. Rogawski, "Representations of $\mathrm{GL}_{n}$ and division algebras over a $p$-adic field", Duke Math. J. 50 (1983), 161-196.

[S-S] P. Schneider et U. StuhleR, "The cohomology of p-adic symmetric spaces", Inv. Math. 105 (1991), 47-122.

[SGA4] Grothendieck, A. ET AL., "SGA 4: Théorie des topos et cohomologie étale des schémas", Lect. Notes in Math. 305 (1973), Springer-Verlag.

[SGA5] Grothendieck, A. ET AL., "SGA 5 : Cohomologie $\ell$-adique et Fonctions L", Lect. Notes in Math. 589 (1977), Springer-Verlag.

[T] J. TATE, "Rigid analytic spaces", Inventiones Math. 12 (1971), 257-289. 
[W] A. WeIL, "Basic Number theory", Springer, Berlin (1985).

[Zi1] TH. ZINK, "Isogenien Formaler Gruppen über einem Lokal Noetherschen Schema", Math. Nachr 99 (1980), 273-283.

[Zi2] Th. Zink, "Cartiertheorie Kommutativer Formaler Gruppen", Teubner-Texte zur Mathematik, Leipzig, Teubner (1984).

décembre 2004

Thomas Hausberger, I3M - UMR CNRS 5149, cc 051, Université Montpellier II, Place Eugène Bataillon, 34095 Montpellier Cedex 5, FRANCE • E-mail : hausberg@math.univ-montp2.fr Url : http://www.math.univ-montp2.fr/ hausberg 